\title{
Nutritional modulation of intestinal inflammation
}

Citation for published version (APA):

Schepens, M. A. A. (2011). Nutritional modulation of intestinal inflammation. [Doctoral Thesis, Maastricht University]. Universiteit Maastricht. https://doi.org/10.26481/dis.20110318ms

Document status and date:

Published: 01/01/2011

DOI:

10.26481/dis.20110318ms

Document Version:

Publisher's PDF, also known as Version of record

\section{Please check the document version of this publication:}

- A submitted manuscript is the version of the article upon submission and before peer-review. There can be important differences between the submitted version and the official published version of record.

People interested in the research are advised to contact the author for the final version of the publication, or visit the DOI to the publisher's website.

- The final author version and the galley proof are versions of the publication after peer review.

- The final published version features the final layout of the paper including the volume, issue and page numbers.

Link to publication

\footnotetext{
General rights rights.

- You may freely distribute the URL identifying the publication in the public portal. please follow below link for the End User Agreement:

www.umlib.nl/taverne-license

Take down policy

If you believe that this document breaches copyright please contact us at:

repository@maastrichtuniversity.nl

providing details and we will investigate your claim.
}

Copyright and moral rights for the publications made accessible in the public portal are retained by the authors and/or other copyright owners and it is a condition of accessing publications that users recognise and abide by the legal requirements associated with these

- Users may download and print one copy of any publication from the public portal for the purpose of private study or research.

- You may not further distribute the material or use it for any profit-making activity or commercial gain

If the publication is distributed under the terms of Article $25 \mathrm{fa}$ of the Dutch Copyright Act, indicated by the "Taverne" license above, 


\section{Nutritional modulation of intestinal inflammation}


Cover design: Anne van Asseldonk

Printing: GVO drukkers \& vormgevers B.V. | Ponsen \& Looijen

ISBN 978-90-6464-454-2

Nutritional modulation of intestinal inflammation

PhD Thesis Maastricht University (2011), The Netherlands - with summary in Dutch Marloes Schepens (MarloesSchepens@gmail.com)

(C) 2011 M.A.A. Schepens 


\title{
Nutritional modulation of intestinal inflammation
}

\author{
PROEFSCHRIFT \\ ter verkrijging van de graad van doctor aan de Universiteit Maastricht, \\ op gezag van de Rector Magnificus, \\ Prof. mr. G.P.M.F. Mols, \\ volgens het besluit van het College van Decanen, \\ in het openbaar te verdedigen \\ op vrijdag 18 maart 2011 om 12.00 uur \\ door
}

Marloes Anna Adriana Schepens 


\section{Promotor}

Prof. dr. R.-J. M. Brummer

\section{Copromotor}

Dr. I.M.J. Bovee-Oudenhoven (NIZO food research)

\section{Beoordelingscommissie}

Prof. dr. A. Bast (voorzitter)

Prof. dr. E. Hultgren-Hörnquist (Örebro University, Sweden)

Prof. dr. P.B. Soeters

Prof. dr. M. Müller (Wageningen University \& Research centre)

Dr. J. Plat
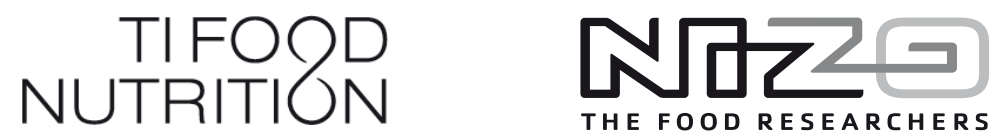

\section{IM Maastricht University}
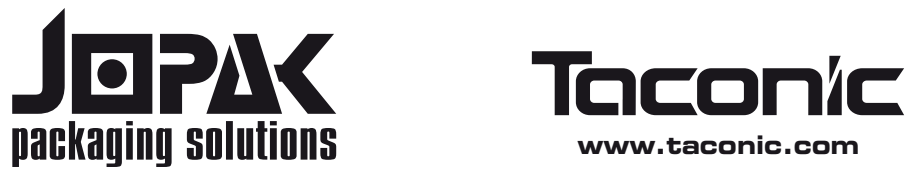

www.taconic.com

The research described in this thesis was performed at NIZO food research. The project was financially supported by Top Institute Food and Nutrition.

The printing of this thesis was financially supported by Top Institute Food and Nutrition, Maastricht University, Jopak and Taconic. 
Voor mijn ouders 



\section{ConTEnTs}

$\begin{array}{lll}\text { Chapter } 1 \quad \text { General introduction } & 9\end{array}$

Chapter 2 Dietary heme adversely affects experimental colitis in rats, 25 despite heat-shock protein induction

Chapter 3 Supplemental calcium attenuates the colitis-related increase in diarrhea, intestinal permeability, and extracellular matrix breakdown in HLA-B27 transgenic rats

Chapter 4 Supplemental antioxidants, in contrast to calcium, do not ameliorate colitis development in HLA-B27 transgenic rats despite extremely low glutathione levels in colonic mucosa

Chapter 5 Dietary calcium decreases but short-chain fructooligosaccharides increase colonic permeability in rats

Chapter 6 The beneficial effect of supplemental calcium on colonic 99 permeability is only affected when dietary phosphate levels are very low

Chapter 7 General discussion

Summary

Samenvatting

Dankwoord

Publicaties en curriculum vitae 



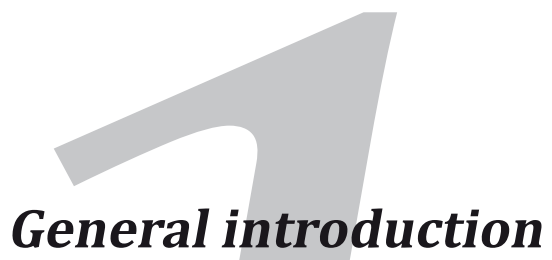




\section{INFLAMMATORY BOWEL DISEASE}

Inflammatory bowel disease (IBD) comprises primarily two disorders of chronic intestinal inflammation: ulcerative colitis and Crohn's disease. These diseases have an intermittent course, with periods of relative disease inactivity or remission, alternating with flare-ups or relapses. The gastrointestinal mucosa of an IBD patient suffers from an uncontrolled inflammation, which can affect any region of the alimentary tract. A key characteristic of ulcerative colitis is that it involves the rectum and may extend proximally to affect part of or the entire colon in an uninterrupted pattern. In contrast, Crohn's disease may affect any site of the gastrointestinal tract and is generally patchy and segmental. However, involvement of the terminal ileum and colon is most common in Crohn's disease. Furthermore, the inflammation is confined to the mucosa in ulcerative colitis patients, while in Crohn's disease, the inflammation is typically transmural ${ }^{(1-2)}$. IBD affects millions of people all over the world, and, as it is a chronic condition currently without a medical cure, it requires a lifetime of health care. Although most patients are able to live normal lives, IBD can cause significant morbidity with symptoms such as diarrhea mixed with blood, abdominal pain, weight loss and extraintestinal manifestations and complications ${ }^{(3)}$.

The incidence and prevalence of IBD varies widely between different countries, northern Europe and North America being high-incidence areas. In Europe, prevalence rates range from 21 to 243 cases per 100,000 persons for ulcerative colitis and from 8 to 214 cases per 100,000 persons for Crohn's disease. At present, a stabilization in the prevalence and incidence of IBD is observed in northern Europe and North America, while incidence is now rising in former low-incidence areas, such as southern Europe and Asia ${ }^{(4)}$. Although the precise etiology of IBD is still unknown, the higher incidence in industrialized regions and the rise in incidence in societies adopting a Westernized lifestyle indicate that there are strong environmental influences on IBD development $t^{(3-4)}$.

In particular diet might play an important role...

\section{PATHOGENESIS OF INFLAMMATORY BOWEL DISEASE}

The etiology and associated pathogenesis of IBD are only beginning to be unveiled. No single agent or mechanism exists that can explain all aspects of IBD. On the contrary, the interaction of various factors contributes to the chronic intestinal inflammation observed in this disorder. The most widely held hypothesis on the pathogenesis of IBD is that the inflammatory process in the intestine results from mucosal barrier dysfunction, microbial factors and dysregulated immune system reactivity in a genetically susceptible host $^{(2,5)}$. Environmental factors, such as diet, are of importance, since they can modulate these three different determinants (Figure 1) ${ }^{(5-6)}$. 


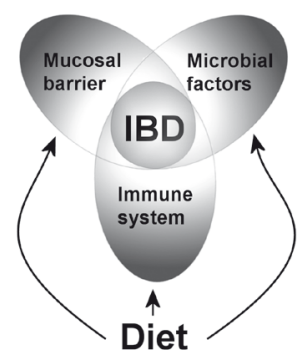

Figure 1: The three different determinants of inflammatory bowel disease (IBD) can be modulated by diet.

\section{Determinant 1: intestinal mucosal barrier}

The intestinal epithelial cell layer constitutes the largest and most important barrier against the external environment (Figure 2). Tight contact between the epithelial cells prevents access of intraluminal toxins, antigens and enteric microbiota to underlying tissue compartments ${ }^{(7-9)}$. The sealing of the space between the cells of the intestinal epithelium is mediated by the apical junctional complex, which is composed of the tight junction. The tight junction is a multiprotein complex which is the principal determinant of mucosal paracellular permeability ${ }^{(10-11)}$. Accumulating evidence shows that paracellular permeability is an important factor contributing to IBD pathogenesis, since an increased permeability intensifies the exposure of the lamina propria to luminal contents, activating an abnormal immune response ${ }^{(9-10)}$. An increased permeability is even suggested to be an indicator of subclinical disease, as it precedes relapse in Crohn's disease $^{(12)}$.

Besides being a tight monolayer, the intestinal epithelium also forms the mucosal barrier by secretion of mucins. Mucins, which are heavily glycosylated proteins, are secreted by goblet cells in the epithelial lining and create a gel that prevents large particles from directly contacting the epithelial cell layer ${ }^{(13)}$. The mucus gel is further strengthened by trefoil factors, which tie the mucin glycoproteins together ${ }^{(14)}$. Changes in the structure and/or quantity of the mucins are also presumed to be involved in the pathogenesis of IBD. For example, it has been observed that none of the biopsies from patients with colonic inflammation showed an intact mucus barrier ${ }^{(15)}$, and deficiency of MUC2, the predominant mucin produced by goblet cells in the large intestine, leads to colonic inflammation in mice ${ }^{(16)}$.

Another process involved in mucosal barrier function is the production of antimicrobials by the intestinal epithelium, such as defensins and bacteriolytic enzymes. Paneth cells are considered to play an important role in the secretion of antimicrobial peptides in IBD. Paneth cell metaplasia has been observed in the colon of IBD patients and this seems to provide additional antimicrobial protection ${ }^{(17-19)}$. 


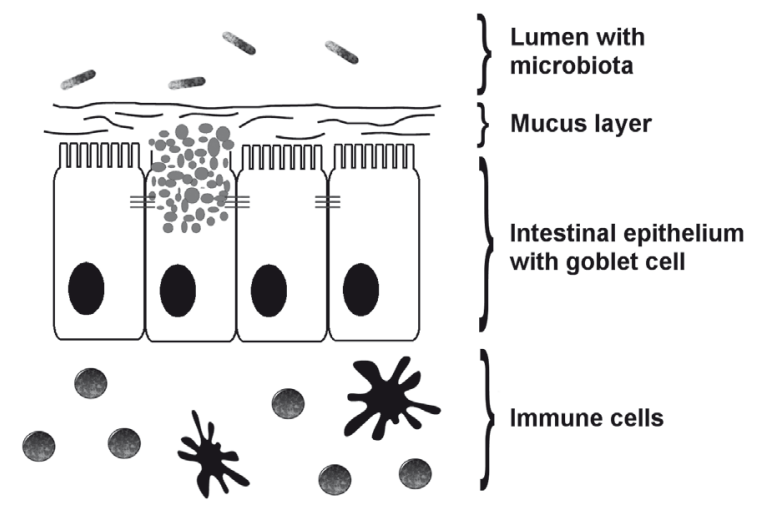

Figure 2: Schematic picture of the colonic epithelial barrier

\section{Determinant 2: microbial factors}

The gastrointestinal tract is colonized by a vast and diverse array of microbes. There is an increase in both concentration and complexity of the microbiota from the stomach and duodenum $\left(10^{2}-10^{3}\right.$ organisms/gram luminal contents) to the cecum and colon $\left(10^{11}-10^{12}\right.$ bacteria/gram luminal contents). The total microbial load of the intestine, $10^{13}-10^{14}$ micro-organisms, collectively contains at least 10 times as many cells as the human body ${ }^{(20-21)}$. The intestinal microbiota provides the host with a variety of benefits, such as supply of energy and nutrients by metabolism of indigestible compounds, defense against colonization by opportunistic pathogens and contribution to the development of the intestinal architecture ${ }^{(22)}$. Moreover, it is suggested that a beneficial partnership has evolved between symbiotic bacteria and the host immune system. The gut microbiome is important for the development of the intestinal immune system, and the immune system in turn shapes the composition of the microbiota ${ }^{(1,22)}$. There is abundant evidence that commensal bacteria are involved in the pathogenesis of IBD. For example, therapeutic benefits of antibiotic treatment have been observed in IBD patients. Also, most experimental IBD models in animals require intestinal bacteria for inflammation to occur. In addition, the intestinal bacterial composition of IBD patients is known to differ from that of healthy controls ${ }^{(1-2,21)}$. It is hypothesized that the equilibrium between inflammation and homeostasis in the gut is due to the composition of the microbiota ${ }^{(22)}$.

\section{Determinant 3: intestinal immune system}

The lamina propria of the intestinal mucosa contains a complex population of immune cells that monitor the luminal contents. The response of the intestinal immune system is challenging, because it must balance immune tolerance to innocuous commensals and defense against pathogens and/or the excessive entry of luminal microbiota ${ }^{(1-2)}$. The immune system consists of the innate and adaptive immunity. Innate microbial sensing by epithelial cells, dendritic cells and macrophages is mediated by pattern- 
recognition receptors such as toll-like receptors and nucleotide oligomerization domain (NOD) proteins. Signaling through these receptors can lead to pro-inflammatory gene expression and activation of the adaptive immune response by recruitment of lymphocytes from the systemic circulation ${ }^{(1,23-24)}$. In the case of an activated immune response, additional innate immune cells migrate into the mucosa, where they generate reactive oxygen species (ROS), possibly resulting in oxidative stress. All the different components of the immune system work in an orchestrated matter to maintain intestinal mucosal homeostasis. However, in IBD there is a disturbance of this balance between immune tolerance and activated defense against intestinal microbiota, leading to aberrant and excessive immunologic responses. This is for example highlighted by the finding that NOD2 is identified as a disease susceptibility gene in Crohn's disease. Bacterial stimulation of disease-associated NOD2 protein results in an exaggerated inflammatory response ${ }^{(5,25)}$. Furthermore, several abnormalities in genes important in immune regulation are associated with IBD, and these defects might lead to overreactivity of the immune system towards normal constituents of the microbiota ${ }^{(2,5)}$. Additionally, production of proinflammatory cytokines and chemokines is enhanced in IBD, and expression of adhesion molecules is increased ${ }^{(2,5)}$.

\section{NUTRITIONAL MODULATION OF INTESTINAL INFLAMMATION}

Nutrition may play an important role in both the pathogenesis and treatment of IBD. As described above, the geographical variation in incidence of IBD accompanied by the adoption of a Westernized lifestyle in the high-incidence regions strongly suggests that diet is a risk factor of IBD. There are indications that Crohn's disease patients have an increased sugar consumption; decreased consumption of fruit and vegetables has been found among IBD patients; and also dietary fiber consumption has been implicated in the risk of IBD. However, several methodological limitations exist in these epidemiologic studies, so very little conclusive evidence is present on the role of specific dietary components in IBD development ${ }^{(26-27)}$.

On the other hand, IBD itself has an impact on the nutritional status of the patient. Many IBD patients suffer from malnutrition. Factors involved in the development of malnutrition in IBD are inadequate oral intake, malabsorption, increased intestinal loss of nutrients, increased calorie needs, and side-effects of medication ${ }^{(26-30)}$. Therefore, nutritional support to correct the nutrient deficiencies is of importance in IBD patients. As already mentioned above, diet is an important environmental factor that can modulate the different determinants of the pathogenesis of IBD. Dietary components can for example alter gene expression of gut cells, influence the composition of the intestinal microbiota, affect intestinal permeability or enhance immune function ${ }^{(31-34)}$. Therefore, nutrition might be an interesting option for IBD patients as a preventive approach to maintain remission as well as a supporting therapeutic strategy ${ }^{(27)}$.

Many nutrients have the potential to influence gut health. The nutrients that are the subject of investigation in this thesis (see end of introduction), are introduced below. 


\section{Heme}

Heme is the dietary component that makes an essential difference between red and white meat. Heme is the iron-containing porphyrin pigment of red meat. Epidemiology suggests that high intake of meat, in particular red meat, increases the likelihood of relapse for ulcerative colitis patients ${ }^{(35)}$. High consumption of red meat, in contrast to white meat, is also associated with an increased risk of sporadic colorectal cancer ${ }^{(36)}$ and it is hypothesized that heme is the component responsible. Dietary heme (but not iron) increases luminal cytotoxicity of the large intestine and causes damage to the colonic epithelium, resulting in compensatory hyperproliferation ${ }^{(37)}$. However, heme is also known as the primary inducer and substrate of heme-oxygenase-1 (HO-1). HO-1 converts heme into possible cytoprotective reaction products ${ }^{(38)}$. HO-1, also known as heat shock protein (HSP) 32, is a member of the HSPs. Inducible HSPs are produced in response to several kinds of stress and serve to stabilize other proteins and preserve their function ${ }^{(39-40)}$. Induction of HSPs has been suggested as a target for amelioration of colitis ${ }^{(38,41-46)}$. Thus, it can be speculated that dietary heme may have dichotomous effects in the lumen of the colon.

\section{Calcium}

Calcium is an important mineral of a healthy diet. Dairy products are a well-known and quantitatively important source of calcium, but calcium is also present in several vegetables, nuts and seeds. In the public domain, supplemental calcium is often interrelated with the prevention of osteoporosis. Furthermore, in several controlled studies, it has been shown that dietary calcium is important in protection against intestinal infections by foodborne bacterial pathogens, both in rats and in humans ${ }^{(47-49)}$. Besides increasing the resistance to gut infections, calcium has displayed cytoprotective effects in several studies in the field of colon carcinogenesis. Importantly, Baron et al showed a reduced risk of colorectal adenoma recurrence due to calcium supplementation in a well-designed clinical trial ${ }^{(50)}$. Upon dietary intake, an insoluble calcium phosphate complex is formed in the small intestine, which can precipitate irritating bile acids and fatty acids. In this way, supplemental calcium is able to reduce the cytotoxicity of the fecal stream. This decrease in luminal cytotoxicity reduces damage to delicate intestinal epithelial cells, which reinforces mucosal integrity ${ }^{(51-52)}$. Furthermore, calcium can influence the intestinal microbiota ${ }^{(53)}$. Despite some studies on calcium and bone health in IBD patients, the influence of calcium on intestinal inflammation has hardly been investigated. At least two studies have reported on possible protective effects of dietary calcium on intestinal inflammation. However, Zhu and co-workers mainly focused on vitamin $\mathrm{D}$ and did not go into much detail about the effect of calcium ${ }^{(54)}$, while results of Pele et al should be interpreted with caution as a direct interaction between calcium and the chemical applied to induce colitis cannot be excluded ${ }^{(55)}$. Based on the generic protective effects of calcium on gut health, it is worthwhile to further explore the potentially beneficial effects calcium may have on intestinal inflammation.

\section{Antioxidants}

Besides the antioxidants which are synthesized by the human body itself, for example 
superoxide dismutase enzymes and glutathione, antioxidants are also found in several foods, such as vegetables, fruits and nuts. Dietary antioxidants include vitamin E, which is a well-known fat-soluble vitamin, vitamin C, a water-soluble vitamin, and polyphenolic antioxidants, such as resveratrol and flavonoids. Numerous antioxidants exist, having all specific roles in the antioxidant network. Moreover, they cooperate in an integrated manner in this network ${ }^{(56)}$. Antioxidants are needed to scavenge and prevent the excessive formation of ROS, which are part of the inflammatory response. This excessive production of ROS is presumed to lead to oxidative stress, which may cause oxidative modification of macromolecules, leading to tissue damage ${ }^{(57-59)}$. The inflamed bowel of IBD patients is infiltrated with leukocytes, generating large amounts of ROS. These ROS are thought to be partly responsible for the intestinal tissue damage seen in IBD. The balance between the amount of ROS produced and the available antioxidative defense is suggested to be impaired in IBD $^{(60)}$. There are indications that antioxidant defenses are reduced in IBD patients ${ }^{(26-27,61)}$. For example, decreased copper/zinc superoxide dismutase activity was found in inflamed tissues of Crohn's disease patients ${ }^{(62)}$. Also, one study reported decreased total peroxyl radical scavenging capacity in inflamed mucosa compared to adjacent noninflamed mucosa, however, no differences were observed in mucosal glutathione and $\alpha$-tocopherol levels ${ }^{(63)}$. For protection against possible oxidative stress in IBD patients, it might be helpful to supplement subjects with dietary antioxidants ${ }^{(59-60,64)}$.

\section{Short-chain fructo-oligosaccharides (scFOS)}

ScFOS are a class of non-digestible oligosaccharides, regarded as a prebiotic. Prebiotics are assumed to be beneficial for the host because they selectively stimulate the growth and/or activity of protective microbiota in the colon, and thus improve host health ${ }^{(65)}$. ScFOS occur naturally in a variety of fruits and vegetables. Daily intake of fructose-based non-digestible carbohydrates has been estimated up to $10 \mathrm{~g}^{(66)}$. This corresponds to $2 \%$ in the human diet, assuming a total food intake of $500 \mathrm{~g}$ dry weight/d. This estimation does not take into account consumption of specific meals and products supplemented with fructose-based carbohydrates, typically 3-10 g per portion ${ }^{(67)}$. Non-digestible carbohydrates, such as scFOS, pass through the small intestine without degradation by host digestive enzymes and therefore reach the large intestine chemically intact. When scFOS enter the colon, they are rapidly hydrolyzed and metabolized by the endogenous microbiota $^{(68)}$. In this way, scFOS change the bacterial composition, which is assumed to be beneficial ${ }^{(65)}$. As microbiota play such an important role in IBD pathogenesis, targeting the intestinal bacteria with prebiotics is suggested to be beneficial for IBD patients ${ }^{(69-70)}$. However, in our lab, consistent adverse effects of scFOS on intestinal resistance to infectious disease have been observed in experiments with rats, particularly when fed a low calcium diet ${ }^{(49,71-72)}$. Therefore, scFOS can also be used as a dietary challenge of the intestinal barrier.

\section{Other nutrients}

Although not included in the research described in this thesis, some other nutrients that are suggested to be able to influence intestinal inflammation, are briefly described 
below.

In the first place, there is considerable and growing research interest in the concept of probiotics. Probiotics are live micro-organisms, which, when consumed in adequate amounts, are thought to promote the health of the host organism by improving the composition of the intestinal microbiota ${ }^{(73-74)}$. For example, there is increasing evidence that pouchitis patients beneficially respond to probiotic intervention ${ }^{(75-76)}$. However, definite clinical evidence for probiotic therapy in IBD is still lacking, despite several in vitro and animal experiments supporting its use in ameliorating gut inflammation ${ }^{\text {(77-78). }}$. Another nutritional supplement receiving great attention regarding possible modulation of gut inflammation in IBD is polyunsaturated fatty acids derived from fish oil. These fatty acids are suggested to ameliorate inflammation by reducing the production of inflammatory mediators originating from arachidonic acid $^{(26,31)}$. Nevertheless, up to now, most studies have not demonstrated significant clinical benefit from supplementation with these fatty acids in IBD patients ${ }^{(76,79)}$. Interestingly, particular polymorphisms might be involved in the sensitivity of a person to the effect of fish oil ${ }^{(80)}$.

A number of other nutrients has been investigated for their ability to ameliorate intestinal inflammation, such as zinc, glutamine and cysteine. Despite substantial research, sufficient evidence for the modulating effects of these nutrients on IBD pathogenesis is (still) lacking. Glutamine, an important energy source for the intestinal epithelial cells, has been shown to influence intestinal barrier properties in animal models; however, no beneficial effect could been identified in IBD $^{(30,81-82)}$. In contrast, zinc supplementation preserved intestinal barrier function in Crohn's disease patients ${ }^{(83)}$, while barrier effects in animal models are lacking ${ }^{(81)}$. Cysteine, which is often the rate-limiting precursor for glutathione synthesis ${ }^{(84)}$ and is an important constituent of the mucus layer, has been investigated in animal models, but there is no conclusive evidence for the influence of this amino acid on intestinal inflammation ${ }^{(82)}$.

\section{INVESTIGATION OF THE NUTRITIONAL MODULATION OF INTESTINAL INFLAMMATION}

It is unethical to study the modulation of intestinal inflammation in humans when hardly any prior evidence is present in the literature concerning the intervention of interest. Therefore, the general approach to investigate efficacy of a nutritional intervention is to start with animal experiments. It is also more convenient to perform strictly controlled experiments in animals, since, for example, completely standardized diets can be used. Furthermore, the inflammatory process in the intestine can be easily investigated in animals by simply taking out their intestine at the end of the experiment, while in humans only mucosal specimens can be obtained by performing endoscopic investigation, which implies substantial discomfort to the subject and often prior cleansing of the colon, which disturbs the natural environment. If a nutrient is shown to be promising in animals, it will be verified in an intervention study with IBD patients. 


\section{Animal models mimicking IBD}

To study intestinal inflammation, several animal models that mimic human IBD are used in the literature. Roughly, they can be divided into genetic models (spontaneous, knock-out and transgenic), adoptive transfer models and models induced by exogenous agents $^{(85-89)}$. Interestingly, and as already mentioned earlier, in most animal models mimicking IBD microbes are necessary to get intestinal inflammation ${ }^{(21)}$. For the research described in this thesis, two different IBD models have been used, which are introduced below.

An easy to apply, reproducible and frequently used model is the trinitrobenzene sulfonic acid (TNBS) colitis model. When TNBS is rectally infused in an animal, colonic inflammation develops almost immediately. This chemically-induced type of colitis has most resemblance to Crohn's disease. A major disadvantage of this model in nutritional research is the strongly decreased food intake of the animals after TNBS administration $^{(85,90)}$.

Another widely used experimental IBD model is the HLA-B27 transgenic rat model. These rats are transgenic for the human HLA-B27 and $\beta 2$ microglobulin genes. They spontaneously develop an inflammatory disease mainly involving the gastrointestinal tract and the colon in particular ${ }^{(91)}$. Also in humans, the HLA-B27 gene is associated with inflammatory disorders ${ }^{(92)}$. Food intake of these rats remains stable during colitis development with time, which is an important condition for nutritional interventions aiming to screen efficacy.

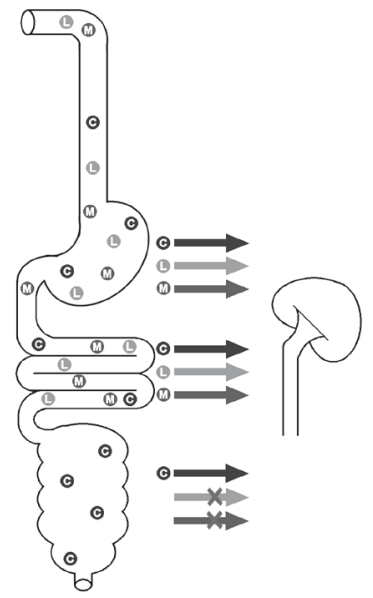

Figure 3: Lactulose (L) and mannitol (M) are instantly degraded by the colonic microbiota. CrEDTA (C) is stable throughout the gut. After passing the epithelium, the probes are cleared by the kidney and excreted in urine. The urinary lactulose/mannitol ratio reflects small intestinal permeability, while urinary CrEDTA represents total intestinal permeability. 


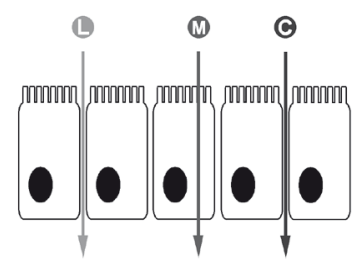

Figure 4: Lactulose (L), mannitol (M) and CrEDTA (C) can be used as permeability probes. Lactulose and CrEDTA pass the epithelium paracellularly, while mannitol passes transcellularly.

\section{Experimental design}

Nutritional modulation of intestinal inflammation has been investigated in this thesis by strictly controlled dietary interventions in animal models of gut inflammation as well as rat models with a challenged intestinal barrier. During and after the animal experiments, several characteristics of intestinal inflammation can be monitored. One of the important subjects of investigation for this thesis was intestinal permeability as a marker for intestinal integrity and barrier function. To study intestinal permeability noninvasively in vivo, permeability probes can be administered orally, such as chromium EDTA (CrEDTA), or mono/disaccharides like lactulose and mannitol. Subsequent measurement of the urinary excretion of the particular permeability marker provides information about the intestinal permeability ${ }^{(7,93)}$. As CrEDTA is stable throughout the intestinal tract and not metabolized, the urinary excretion of this marker provides information regarding total intestinal permeability ${ }^{(94)}$. By choosing permeability probes that have limited exposure to certain parts of the intestinal epithelium, region-specific permeability measurements can be performed ${ }^{(95)}$. For example, the saccharide probes lactulose and mannitol can be used to measure small intestinal permeability, because they are instantly degraded by the colonic microbiota (Figure 3). Since lactulose only passes the gut wall paracellularly and mannitol passes transcellularly, the lactulose/ mannitol ratio is often used to express small intestinal permeability. By using a ratio, possible differences in epithelial surface or transit time can be corrected for (Figure $4)^{(7)}$. So, urine can be collected to quantify intestinal permeability, but also feces can be sampled during the animal experiments performed for the research described in this thesis. Diarrhea, mucins and microbiota can be determined in feces. In addition, at the end of the experiments, the rats were sacrificed and then their colons can be taken out for macroscopic and microscopic evaluation of inflammation symptoms by using a scoring system.

Inflammation can be further quantified in mucosa scraped off from the colon. In particular myeloperoxidase is an important inflammatory marker, and it is often considered the gold standard to quantify inflammation. Other inflammatory markers of interest to measure in colonic mucosa are for example cytokines. Furthermore, acute phase proteins and oxidative stress markers can be investigated in mucosal samples, but also mRNA can be isolated for quantification of differential gene expression. 


\section{RATIONALE AND OUTLINE OF THIS THESIS}

The general hypothesis for the research described in this thesis was that intestinal inflammation can be modulated by nutritional intervention. Subsequently, an attempt was made to gain insight into the mechanisms involved in the nutrient-dependent modulation. These results are relevant for the substantiation of health claims and the development of functional foods. Moreover, scientific knowledge generated with these experiments contributes to further understanding of the processes involved in IBD, but also of other disorders characterized by compromised gut function, like irritable bowel syndrome.

The first study, described in chapter $\mathbf{2}$ of this thesis, investigated the effect of dietary heme on TNBS-induced colitis in rats. Rats were fed 'humanized' Western diets either with or without heme for 10 days, after which colitis was induced. Colitis severity was evaluated and HSPs were quantified to study the influence of heme.

In chapter 3, the effect of supplemental calcium on intestinal inflammation was explored. In this study, HLA-B27 transgenic rats were fed a 'humanized' Western diet containing either a low or high calcium content. CrEDTA was added to the diets to quantify intestinal permeability, and gut inflammation was determined. Furthermore, colonic mucosal gene expression was analyzed using whole-genome microarrays.

The aim of the study described in chapter $\mathbf{4}$ was to investigate whether supplementation of a mixture of antioxidants would ameliorate colitis development in HLA-B27 transgenic rats. It was also investigated whether the dietary combination of antioxidants and calcium has additive protective effects on colitis development, as it was hypothesized that antioxidants might act at mucosa level and calcium predominantly in the gut lumen. HLA-B27 rats were fed a control diet, a diet supplemented with the antioxidants glutathione, vitamin $\mathrm{C}$ and vitamin $\mathrm{E}$, or a diet supplemented with both antioxidants and calcium. Intestinal permeability and several characteristics of intestinal inflammation were determined, and in particular the oxidative stress status. As remarkable findings were observed with regard to the transgenic rat model, an additional animal experiment was included in this study in which young and older HLA-B27 rats were compared.

Since previous studies showed effects of dietary calcium and scFOS on intestinal permeability, chapter $\mathbf{5}$ describes a more mechanistic study, in which it was investigated how and where in the gastrointestinal tract these nutrients exert their influence on intestinal permeability. To study this, rats were fed a 'humanized' Western control diet, or a similar diet supplemented with calcium or scFOS. Lactulose plus mannitol and CrEDTA were added to the diets to measure small and total intestinal permeability respectively. Additionally, Ussing chamber experiments were performed in which colonic tissue was exposed to fecal water of these rats.

Chapter $\mathbf{6}$ concerns another mechanistic study to address whether the beneficial effect of calcium on intestinal permeability depends on its dragging of phosphate into the colon and the subsequent increase in luminal buffering capacity. For this purpose, rats were fed different quantities of calcium and phosphate. Again, CrEDTA was added to 
the diets to quantify intestinal permeability. ScFOS were supplemented to the different diets to challenge the gut barrier.

Finally, in chapter 7, the major findings of this thesis are summarized, discussed and put in a broader perspective. Some concluding remarks on the research performed in this thesis close the chapter.

\section{REFERENCES}

1. Abraham C \& Cho JH (2009) Inflammatory bowel disease. N Engl J Med 361, 2066-2078.

2. Xavier RJ \& Podolsky DK (2007) Unravelling the pathogenesis of inflammatory bowel disease. Nature 448, 427-434.

3. Hanauer SB (2006) Inflammatory bowel disease: epidemiology, pathogenesis, and therapeutic opportunities. Inflamm Bowel Dis 12 Suppl 1, S3-9.

4. Loftus EV, Jr. (2004) Clinical epidemiology of inflammatory bowel disease: Incidence, prevalence, and environmental influences. Gastroenterology 126, 1504-1517.

5. Sartor RB (2006) Mechanisms of disease: pathogenesis of Crohn's disease and ulcerative colitis. Nat Clin Pract Gastroenterol Hepatol 3, 390-407.

6. Danese S, Sans M \& Fiocchi C (2004) Inflammatory bowel disease: the role of environmental factors. Autoimmun Rev 3, 394-400.

7. Arrieta MC, Bistritz L \& Meddings JB (2006) Alterations in intestinal permeability. Gut 55, 1512-1520.

8. Farhadi A, Banan A, Fields J et al. (2003) Intestinal barrier: an interface between health and disease. J Gastroenterol Hepatol 18, 479-497.

9. Groschwitz KR \& Hogan SP (2009) Intestinal barrier function: molecular regulation and disease pathogenesis. J Allergy Clin Immunol 124, 3-20; quiz 21-22.

10. Clayburgh DR, Shen L \& Turner JR (2004) A porous defense: the leaky epithelial barrier in intestinal disease. Lab Invest 84, 282-291.

11. Turner JR (2009) Intestinal mucosal barrier function in health and disease. Nat Rev Immunol 9, 799809.

12. Wyatt J, Vogelsang H, Hubl W et al. (1993) Intestinal permeability and the prediction of relapse in Crohn's disease. Lancet 341, 1437-1439.

13. Einerhand AW, Renes IB, Makkink MK et al. (2002) Role of mucins in inflammatory bowel disease: important lessons from experimental models. Eur J Gastroenterol Hepatol 14, 757-765.

14. Taupin D \& Podolsky DK (2003) Trefoil factors: initiators of mucosal healing. Nat Rev Mol Cell Biol 4, 721-732.

15. Swidsinski A, Loening-Baucke V, Theissig F et al. (2007) Comparative study of the intestinal mucus barrier in normal and inflamed colon. Gut 56, 343-350.

16. Van der Sluis M, De Koning BA, De Bruijn AC et al. (2006) Muc2-deficient mice spontaneously develop colitis, indicating that MUC2 is critical for colonic protection. Gastroenterology 131, 117-129.

17. Cunliffe RN \& Mahida YR (2004) Expression and regulation of antimicrobial peptides in the gastrointestinal tract. J Leukoc Biol 75, 49-58.

18. Elphick DA \& Mahida YR (2005) Paneth cells: their role in innate immunity and inflammatory disease. Gut 54, 1802-1809.

19. Wehkamp J, Schmid M \& Stange EF (2007) Defensins and other antimicrobial peptides in inflammatory bowel disease. Curr Opin Gastroenterol 23, 370-378.

20. Abt MC \& Artis D (2009) The intestinal microbiota in health and disease: the influence of microbial products on immune cell homeostasis. Curr Opin Gastroenterol 25, 496-502.

21. Sartor RB (2008) Microbial influences in inflammatory bowel diseases. Gastroenterology 134, 577-594.

22. Round JL \& Mazmanian SK (2009) The gut microbiota shapes intestinal immune responses during health and disease. Nat Rev Immunol 9, 313-323.

23. Karin M, Lawrence T \& Nizet V (2006) Innate immunity gone awry: linking microbial infections to chronic inflammation and cancer. Cell 124, 823-835.

24. Macdonald TT \& Monteleone G (2005) Immunity, inflammation, and allergy in the gut. Science 307, 1920-1925. 
25. Abreu MT, Fukata M \& Arditi M (2005) TLR signaling in the gut in health and disease. J Immunol 174, 4453-4460.

26. Geerling BJ, Stockbrugger RW \& Brummer RJ (1999) Nutrition and inflammatory bowel disease: an update. Scand J Gastroenterol Suppl 230, 95-105.

27. Ling SC \& Griffiths AM (2000) Nutrition in inflammatory bowel disease. Curr Opin Clin Nutr Metab Care 3, 339-344.

28. Campos FG, Waitzberg DL, Teixeira MG et al. (2002) Inflammatory bowel diseases: principles of nutritional therapy. Rev Hosp Clin Fac Med Sao Paulo 57, 187-198.

29. Han PD, Burke A, Baldassano RN et al. (1999) Nutrition and inflammatory bowel disease. Gastroenterol Clin North Am 28, 423-443, ix.

30. Wild GE, Drozdowski L, Tartaglia C et al. (2007) Nutritional modulation of the inflammatory response in inflammatory bowel disease--from the molecular to the integrative to the clinical. World J Gastroenterol 13, 1-7.

31. Chapman-Kiddell CA, Davies PS, Gillen L et al. (2009) Role of diet in the development of inflammatory bowel disease. Inflamm Bowel Dis.

32. Ferguson LR, Shelling AN, Browning BL et al. (2007) Genes, diet and inflammatory bowel disease. Mutat Res 622, 70-83.

33. Goh J \& O'Morain CA (2003) Review article: nutrition and adult inflammatory bowel disease. Aliment Pharmacol Ther 17, 307-320.

34. Rowlands BJ \& Gardiner KR (1998) Nutritional modulation of gut inflammation. Proc Nutr Soc 57, 395401.

35. Jowett SL, Seal CJ, Pearce MS et al. (2004) Influence of dietary factors on the clinical course of ulcerative colitis: a prospective cohort study. Gut 53, 1479-1484.

36. Chao A, Thun MJ, Connell CJ et al. (2005) Meat consumption and risk of colorectal cancer. Jama 293, 172182.

37. Sesink AL, Termont DS, Kleibeuker JH et al. (1999) Red meat and colon cancer: the cytotoxic and hyperproliferative effects of dietary heme. Cancer Res 59, 5704-5709.

38. Otterbein LE, Soares MP, Yamashita K et al. (2003) Heme oxygenase-1: unleashing the protective properties of heme. Trends Immunol 24, 449-455.

39. Otaka M, Odashima M \& Watanabe S (2006) Role of heat shock proteins (molecular chaperones) in intestinal mucosal protection. Biochem Biophys Res Commun 348, 1-5.

40. Petrof EO, Ciancio MJ \& Chang EB (2004) Role and regulation of intestinal epithelial heat shock proteins in health and disease. Chin J Dig Dis 5, 45-50.

41. Naito Y, Takagi T \& Yoshikawa T (2004) Heme oxygenase-1: a new therapeutic target for inflammatory bowel disease. Aliment Pharmacol Ther 20 Suppl 1, 177-184.

42. Odashima M, Otaka M, Jin M et al. (2006) Zinc l-carnosine protects colonic mucosal injury through induction of heat shock protein 72 and suppression of NF-kappaB activation. Life Sci 79, 2245-2250.

43. Ohkawara T, Nishihira J, Takeda $\mathrm{H}$ et al. (2006) Protective effect of geranylgeranylacetone on trinitrobenzene sulfonic acid-induced colitis in mice. Int J Mol Med 17, 229-234.

44. Ohkawara T, Takeda H, Kato K et al. (2005) Polaprezinc (N-(3-aminopropionyl)-L-histidinato zinc) ameliorates dextran sulfate sodium-induced colitis in mice. Scand J Gastroenterol 40, 1321-1327.

45. Otani S, Otaka M, Jin M et al. (1997) Effect of preinduction of heat shock proteins on acetic acid-induced colitis in rats. Dig Dis Sci 42, 833-846.

46. Sikora A \& Grzesiuk E (2007) Heat shock response in gastrointestinal tract. J Physiol Pharmacol 58 Suppl 3, 43-62.

47. Bovee-Oudenhoven IM, Lettink-Wissink ML, Van Doesburg W et al. (2003) Diarrhea caused by enterotoxigenic Escherichia coli infection of humans is inhibited by dietary calcium. Gastroenterology $125,469-476$.

48. Bovee-Oudenhoven IM, Termont DS, Weerkamp AH et al. (1997) Dietary calcium inhibits the intestinal colonization and translocation of Salmonella in rats. Gastroenterology 113, 550-557.

49. Ten Bruggencate SJ, Bovee-Oudenhoven IM, Lettink-Wissink ML et al. (2004) Dietary fructooligosaccharides and inulin decrease resistance of rats to salmonella: protective role of calcium. Gut 53, 530-535.

50. Baron JA, Beach M, Mandel JS et al. (1999) Calcium supplements for the prevention of colorectal 
adenomas. Calcium Polyp Prevention Study Group. N Engl J Med 340, 101-107.

51. Govers MJ, Termont DS, Lapre JA et al. (1996) Calcium in milk products precipitates intestinal fatty acids and secondary bile acids and thus inhibits colonic cytotoxicity in humans. Cancer Res 56, 3270-3275.

52. Lapre JA, De Vries HT, Koeman JH et al. (1993) The antiproliferative effect of dietary calcium on colonic epithelium is mediated by luminal surfactants and dependent on the type of dietary fat. Cancer Res 53, 784-789.

53. Bovee-Oudenhoven IM, Wissink ML, Wouters JT et al. (1999) Dietary calcium phosphate stimulates intestinal lactobacilli and decreases the severity of a salmonella infection in rats. J Nutr 129, 607-612.

54. Zhu Y, Mahon BD, Froicu M et al. (2005) Calcium and 1 alpha,25-dihydroxyvitamin D3 target the TNFalpha pathway to suppress experimental inflammatory bowel disease. Eur J Immunol 35, 217-224.

55. Pele LC, Thoree V, Mustafa F et al. (2007) Low dietary calcium levels modulate mucosal caspase expression and increase disease activity in mice with dextran sulfate sodium induced colitis. J Nutr 137, 2475-2480.

56. Blomhoff R, Carlsen MH, Andersen LF et al. (2006) Health benefits of nuts: potential role of antioxidants. Br J Nutr 96 Suppl 2, S52-60.

57. Fang YZ, Yang S \& Wu G (2002) Free radicals, antioxidants, and nutrition. Nutrition 18, 872-879.

58. Halliwell B (1996) Antioxidants in human health and disease. Annu Rev Nutr 16, 33-50.

59. Kruidenier L \& Verspaget HW (2002) Review article: oxidative stress as a pathogenic factor in inflammatory bowel disease--radicals or ridiculous? Aliment Pharmacol Ther 16, 1997-2015.

60. Kruidenier L \& Verspaget HW (1998) Antioxidants and mucosa protectives: realistic therapeutic options in inflammatory bowel disease? Mediators Inflamm 7, 157-162.

61. Kruidenier L, Kuiper I, Van Duijn W et al. (2003) Imbalanced secondary mucosal antioxidant response in inflammatory bowel disease. J Pathol 201,17-27.

62. Lih-Brody L, Powell SR, Collier KP et al. (1996) Increased oxidative stress and decreased antioxidant defenses in mucosa of inflammatory bowel disease. Dig Dis Sci 41, 2078-2086.

63. Buffinton GD \& Doe WF (1995) Depleted mucosal antioxidant defences in inflammatory bowel disease. Free Radic Biol Med 19, 911-918.

64. Miralles-Barrachina O, Savoye G, Belmonte-Zalar L et al. (1999) Low levels of glutathione in endoscopic biopsies of patients with Crohn's colitis: the role of malnutrition. Clin Nutr 18, 313-317.

65. Gibson GR \& Roberfroid MB (1995) Dietary modulation of the human colonic microbiota: introducing the concept of prebiotics. J Nutr 125, 1401-1412.

66. Coussement PA (1999) Inulin and oligofructose: safe intakes and legal status. J Nutr 129, 1412S-1417S.

67. van Loo J, Coussement P, de Leenheer L et al. (1995) On the presence of inulin and oligofructose as natural ingredients in the western diet. Crit Rev Food Sci Nutr 35, 525-552.

68. Andersson HB, Ellegard LH \& Bosaeus IG (1999) Nondigestibility characteristics of inulin and oligofructose in humans. J Nutr 129, 1428S-1430S.

69. Leenen CH \& Dieleman LA (2007) Inulin and oligofructose in chronic inflammatory bowel disease. J Nutr 137, 2572S-2575S.

70. Looijer-van Langen MA \& Dieleman LA (2009) Prebiotics in chronic intestinal inflammation. Inflamm Bowel Dis 15, 454-462.

71. Ten Bruggencate SJ, Bovee-Oudenhoven IM, Lettink-Wissink ML et al. (2003) Dietary fructooligosaccharides dose-dependently increase translocation of salmonella in rats. J Nutr 133, 2313-2318.

72. Ten Bruggencate SJ, Bovee-Oudenhoven IM, Lettink-Wissink ML et al. (2005) Dietary fructooligosaccharides increase intestinal permeability in rats. J Nutr 135, 837-842.

73. Guarner F \& Malagelada JR (2003) Gut flora in health and disease. Lancet 361, 512-519.

74. Preidis GA \& Versalovic J (2009) Targeting the human microbiome with antibiotics, probiotics, and prebiotics: gastroenterology enters the metagenomics era. Gastroenterology 136, 2015-2031.

75. Gionchetti P, Rizzello F, Tambasco R et al. (2008) Which therapies are advisable in pouchitis? Inflamm Bowel Dis 14 Suppl 2, S241-242.

76. Penner R, Fedorak RN \& Madsen KL (2005) Probiotics and nutraceuticals: non-medicinal treatments of gastrointestinal diseases. Curr Opin Pharmacol 5, 596-603.

77. Hormannsperger G \& Haller D (2010) Molecular crosstalk of probiotic bacteria with the intestinal immune system: clinical relevance in the context of inflammatory bowel disease. Int J Med Microbiol 300, 63-73. 
78. Rioux KP, Madsen KL \& Fedorak RN (2005) The role of enteric microflora in inflammatory bowel disease: human and animal studies with probiotics and prebiotics. Gastroenterol Clin North Am 34, 465-482, ix.

79. MacLean CH, Mojica WA, Newberry SJ et al. (2005) Systematic review of the effects of n-3 fatty acids in inflammatory bowel disease. Am J Clin Nutr 82, 611-619.

80. Grimble RF, Howell WM, O'Reilly G et al. (2002) The ability of fish oil to suppress tumor necrosis factor alpha production by peripheral blood mononuclear cells in healthy men is associated with polymorphisms in genes that influence tumor necrosis factor alpha production. Am J Clin Nutr 76, 454459.

81. Hering NA \& Schulzke JD (2009) Therapeutic options to modulate barrier defects in inflammatory bowel disease. Dig Dis 27, 450-454.

82. Coeffier M, Marion-Letellier R \& Dechelotte P (2010) Potential for amino acids supplementation during inflammatory bowel diseases. Inflamm Bowel Dis 16, 518-524.

83. Sturniolo GC, Di Leo V, Ferronato A et al. (2001) Zinc supplementation tightens "leaky gut" in Crohn's disease. Inflamm Bowel Dis 7, 94-98.

84. Meister A (1991) Glutathione deficiency produced by inhibition of its synthesis, and its reversal; applications in research and therapy. Pharmacol Ther 51,155-194.

85. Elson CO, Sartor RB, Tennyson GS et al. (1995) Experimental models of inflammatory bowel disease. Gastroenterology 109, 1344-1367.

86. Hibi T, Ogata H \& Sakuraba A (2002) Animal models of inflammatory bowel disease. J Gastroenterol 37, 409-417.

87. Hoffmann JC, Pawlowski NN, Kuhl AA et al. (2002) Animal models of inflammatory bowel disease: an overview. Pathobiology 70, 121-130.

88. Jurjus AR, Khoury NN \& Reimund JM (2004) Animal models of inflammatory bowel disease. J Pharmacol Toxicol Methods 50, 81-92.

89. Reimann J, Rudolphi A \& Claesson MH (1995) Novel experimental approaches in the study of the immunopathology in inflammatory bowel disease. J Mol Med 73, 133-140.

90. Morris GP, Beck PL, Herridge MS et al. (1989) Hapten-induced model of chronic inflammation and ulceration in the rat colon. Gastroenterology 96, 795-803.

91. Sartor RB (2000) Colitis in HLA-B27/beta 2 microglobulin transgenic rats. Int Rev Immunol 19, 39-50.

92. Hammer RE, Maika SD, Richardson JA et al. (1990) Spontaneous inflammatory disease in transgenic rats expressing HLA-B27 and human beta 2m: an animal model of HLA-B27-associated human disorders. Cell 63, 1099-1112.

93. Bjarnason I, MacPherson A \& Hollander D (1995) Intestinal permeability: an overview. Gastroenterology 108, 1566-1581.

94. Aabakken L (1989) Cr-ethylenediaminetetraacetic acid absorption test. Methodologic aspects. Scand J Gastroenterol 24, 351-358.

95. Meddings JB \& Gibbons I (1998) Discrimination of site-specific alterations in gastrointestinal permeability in the rat. Gastroenterology 114, 83-92. 
Chapter 1

I Chapter 1

| 24 


\section{Dietary heme adversely affects experimental colitis in rats, despite heat-shock protein induction}

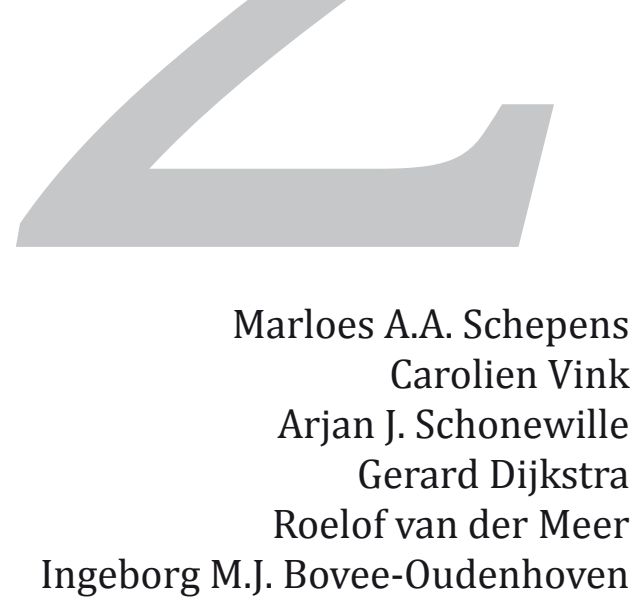

Ingeborg M.J. Bovee-Oudenhoven

Nutrition. 2010 Aug 11. In press 


\section{Abstract}

Objective: Research on dietary modulation of inflammatory bowel disease is in its infancy. Dietary heme, mimicking red meat, is cytotoxic to colonic epithelium and thus may aggravate colitis. Alternatively, heme-induced colonic stress might also result in potential protective heat-shock proteins (HSPs). Therefore, we investigated the effect of dietary heme on trinitrobenzene sulfonic acid (TNBS)-induced colitis in rats.

Methods: Rats were fed a high-fat control diet or a similar diet supplemented with heme. After dietary adaptation, rats were rectally infused with TNBS for colitis induction or saline for sham treatment. Colitis severity was evaluated and several markers were quantified in colonic mucosa isolated $1 \mathrm{wk}$ after colitis induction. Furthermore, cytotoxicity of fecal water and serum $\alpha$-1-acid glycoprotein were measured.

Results: Dietary heme increased cytotoxicity of the fecal water. Heme-fed shamtreated rats had higher colonic HSP-25 and heme-oxygenase-1 mRNA levels, which was confirmed by immunohistochemistry. HSP induction by heme was associated with decreased protein levels of myeloperoxidase and interleukin- $1 \beta$ after subsequent TNBS infusion. However, no dietary effects were observed on histologic colitis score. Furthermore, body weight gain, colon length, and food intake were lower and $\alpha-1-$ acid glycoprotein concentrations were higher in heme-fed colitic rats. In addition, somatostatin, involved in mucosal repair, was not changed with TNBS infusion in hemefed rats.

Conclusion: Dietary heme adversely affects colitis, despite HSP induction. We speculate that the irritating influence of dietary heme, being continuously present in the colon, impairs recovery after colitis induction. A diet high in red meat might be a risk factor for inflammatory bowel disease development. 


\section{INTRODUCTION}

Inflammatory bowel diseases (IBDs) are chronic relapsing and remitting disorders, characterized by intestinal inflammation. The exact etiology of IBD is still not understood, but the disease is considered multifactorial. Various determinants are involved in the pathogenesis, namely the gut mucosal barrier, the immune system, and microbial factors ${ }^{(1,2)}$. Nutrition is of particular importance because it can modulate these determinants. Therefore, dietary modulation of IBD might be an attractive option to prevent relapse and decrease disease symptoms ${ }^{(3)}$.

We are interested in the colonic effects of dietary heme, which is the iron-containing porphyrin pigment of red meat. Epidemiology suggests that high meat intake, in particular red meat, increases the likelihood of relapse for patients with $\operatorname{colitis}^{(4)}$. High consumption of red meat is also associated with an increased risk of sporadic colorectal cancer $^{(5)}$ and it is hypothesized that heme is the component responsible. Sesink et al. ${ }^{(6)}$ showed that dietary heme (but not iron) increases luminal cytotoxicity and causes damage to the colonic epithelium, resulting in compensatory hyperproliferation. Regarding these data, we hypothesize that dietary heme aggravates colitis development. However, heme is also known as the primary inducer and substrate of heme-oxygenase-1 (HO-1). HO-1 converts heme into possible cytoprotective reaction products ${ }^{(7)}$. $\mathrm{HO}-1$, also known as heat-shock protein (HSP)-32, is a member of the HSPs. Inducible HSPs are produced in response to several kinds of stress and serve to stabilize other proteins and preserve their function ${ }^{(8,9)}$. Induction of HSPs has been suggested as a target for amelioration of colitis ${ }^{(7,10-15)}$. Accordingly, as a response to heme-induced colonic stress, it can be speculated that HSPs are evoked in colonic mucosa. Therefore, the irritating effect of dietary heme on colonic mucosa might lead to protection against subsequent inflammation by the induction of HSPs.

Despite possible HSP induction by dietary heme, we hypothesized that heme irritates the intestinal epithelium, subsequently aggravating colitis development. To investigate this, rats were fed a control diet or a similar diet supplemented with heme, after which mucosal inflammation was induced by trinitrobenzene sulfonic acid (TNBS), or saline was infused for sham treatment. The rat TNBS model is a widely used animal model in experimental IBD research ${ }^{(16,17)}$. Inducible HSPs were quantified in the colon and colitis severity was evaluated systemically and locally.

\section{MATERIALS AND METHODS}

\section{Experimental design: Animals, diets, and colitis induction}

The experimental protocol was approved by the animal welfare committee of Wageningen University and Research Centre (Wageningen, The Netherlands). Specific pathogen-free outbred male Wistar rats (WU, Harlan, Horst, The Netherlands), 7 to 8 wk old, with a mean body weight of $270 \mathrm{~g}$, were housed individually in metabolic cages. Animals were kept in a temperature- and humidity-controlled environment in a 12-h light/dark cycle. Rats were fed a purified diet that contained in the control situation 
(per kilogram) $200 \mathrm{~g}$ of acid casein, $326 \mathrm{~g}$ of corn starch,174 g of glucose,160 g of palm oil, $40 \mathrm{~g}$ of corn oil, $50 \mathrm{~g}$ of cellulose, and $5.16 \mathrm{~g}$ of $\mathrm{CaHPO}_{4} 2 \mathrm{H}_{2} \mathrm{O}$ (corresponding to 30 $\mathrm{mmol}$ of calcium/kg of diet; Sigma-Aldrich, St. Louis, MO, USA). Vitamins and minerals (other than calcium) were added to the diets according to the 1993 recommendations of the American Institute of Nutrition ${ }^{(18)}$. Compared with normal rat diets ${ }^{(18)}$, the diets were relatively low in calcium and had a high fat content (39\% energy) to better mimic the composition of a Western human $\operatorname{diet}^{(19)}$. The experimental diet was supplemented with heme ( $0.5 \mathrm{mmol}$ of heme/kg of diet; Sigma-Aldrich) at the expense of glucose. Rats were fed ad libitum throughout the entire study. Food was replaced each day just before dark (eating period). Food intake was recorded daily and animal weight at least once every $3 \mathrm{~d}$. Rats were acclimatized to the housing and dietary conditions for $12 \mathrm{~d}$ before colitis was induced.

Animals under light isoflurane anesthesia were given a single rectal infusion with 0.25 $\mathrm{mL}$ of $50 \%$ ethanol containing $20 \mathrm{mg}$ of TNBS (Sigma-Aldrich) to induce colitis $(\mathrm{n}=11$ per dietary group) or $0.25 \mathrm{~mL}$ of saline for sham treatment $(\mathrm{n}=6$ per dietary group) through a catheter inserted $8 \mathrm{~cm}$ from the anus into the colon ${ }^{(16)}$. On day 7 after TNBS or saline infusion, rats were sacrificed by carbon dioxide inhalation. Orbital blood was obtained for preparation of serum. The colon was taken out, washed in saline, and weight and length were measured. The distal $8 \mathrm{~cm}$ of the colon was excised. A 1-cm piece of a macroscopically affected region was cut out and stored in $10 \%$ neutral buffered formalin for histology. The remaining $7 \mathrm{~cm}$ was longitudinally excised. Severity of colitis was evaluated macroscopically from 0 to 10 using the scoring system of Wallace and Keenan $^{(20)}$. Subsequently, the mucosa was scraped off using a spatula and immediately frozen in liquid nitrogen until further processing and analyses.

\section{Quantitative real-time polymerase chain reaction of mucosal samples}

Frozen colonic mucosal scrapings were manually crushed in liquid nitrogen using a precooled mortar and pestle. After homogenization, part of the powder was used for protein analyses (see below), and RNA was isolated from the remaining portion using TRIzol reagent (Invitrogen, Carlsbad, CA, USA) according to the manufacturer's protocol. RNA was purified with an RNeasy column (Qiagen, Venlo, The Netherlands) and quantified spectrophotometrically by measuring $A_{260}$. Subsequently, $1 \mu$ g of RNA was reverse-transcribed to obtain complementary DNA (cDNA) using reverse transcriptase and oligo $\mathrm{d}(\mathrm{T})_{16}$ primer (Applied Biosystems, Foster City, CA, USA) at $48^{\circ} \mathrm{C}$ for $30 \mathrm{~min}$, followed by 5 min at $95^{\circ} \mathrm{C}$.

Differential expression of individual genes was assessed by quantitative realtime polymerase chain reaction (Q-PCR), using primers presented in Table 1, except for somatostatin, which was measured by a Taqman gene expression assay according to the manufacturer's instructions (Applied Biosystems). For every other reaction, $2 \mu \mathrm{L}$ of 10 fold diluted cDNA was added to $23 \mu \mathrm{L}$ of premix, containing $1 \mu \mathrm{L}$ of forward primer (10 $\mu \mathrm{mol} / \mathrm{L}), 1 \mu \mathrm{L}$ of reverse primer $(10 \mu \mathrm{mol} / \mathrm{L}), 8.5 \mu \mathrm{L}$ of RNase-free water, and $12.5 \mu \mathrm{L}$ of Power SYBR green PCR Master Mix (Applied Biosystems). The samples were incubated for $10 \mathrm{~min}$ at $95^{\circ} \mathrm{C}$, followed by 40 amplification cycles. Each cycle consisted of $15 \mathrm{~s}$ at $95^{\circ} \mathrm{C}$ and 1 min at the annealing temperature listed in Table 1 , using the 7500 Fast Real- 
Table 1: Primer information

\begin{tabular}{lllll}
\hline Gene & $\begin{array}{l}\text { Accession } \\
\text { number }\end{array}$ & Forward primer $\left(5^{\prime} \rightarrow 3^{\prime}\right)$ & Reverse primer $\left(5 \rightarrow 3^{\prime}\right)$ & $\begin{array}{l}\text { Annealing } \\
\text { temperature }\left({ }^{\circ} \mathrm{C}\right)\end{array}$ \\
\hline HSP-25 & NM_031970 & GCGCTCAACCGGCAACTC & AGCAAGCTGAAGGCTTCTAC & 59 \\
HO-1 & NM_012580 & CACGCATATACCCGCTACCT & AAGGCGGTCTTAGCCTCTTC & 60 \\
HSP-70-1 & NM_212504 & TTTCTGGCTCTCAGGGTGTT & CTGTACACAGGGTGGCAGTG & 61 \\
Aldolase & NM_012495 & ATGCCCCACCCATACCCAGCACT & AGCAGCAGTTGGCGGTAGAAGCG & 60 \\
GAPDH & XM_574168 & TGCACCACCAACTGCTTA & GGATGCAGGGATGATGTTC & 58 \\
\hline
\end{tabular}

GAPDH, glyceraldehyde-3-phosphate dehydrogenase; HO-1, heme-oxygenase-1; HSP-25, heat-shock protein-25; HSP-70-1, heat-shock protein-70-1

Time PCR System (Applied Biosystems). The run was completed with melting curve analysis for every PCR product. A standard curve for each particular gene was run with every assay using serial dilutions of a reference sample (cDNA synthesized from a pooled RNA sample). Messenger RNA levels were determined from the appropriate standard curve, and these data were normalized against the housekeeping genes aldolase and glyceraldehyde-3-phosphate dehydrogenase as indicated.

\section{Mucosal protein analyses}

Part of the frozen homogenized colonic scrapingswas suspended in a solution containing $200 \mathrm{mmol} / \mathrm{L}$ of sucrose, $20 \mathrm{mmol} / \mathrm{L}$ of Tris, $1 \mathrm{mmol} / \mathrm{L}$ of dithiothreitol, and protease inhibitors for analyses of myeloperoxidase (MPO), interleukin-1 $\beta$ (IL-1 $\beta$ ), and total protein content. MPO and IL-1 $\beta$ were determined using enzymelinked immunosorbent assay kits (MPO: Hycult Biotechnology b.v., Uden, The Netherlands; IL-1ß: Biosource, Camarillo, CA, USA) according to the manufacturers' instructions. Total protein content of the scrapings was measured spectrophotometrically using the bicinchoninic acid (BCA) protein assay kit (Uptima, Montluçon, France) with bovine serum albumin (Sigma-Aldrich) as a standard.

\section{Analyses of fecal water}

Feces were quantitatively collected during days 6 and 7 after rectal infusion of TNBS or saline and freeze-dried. Fecal water was prepared as described previously ${ }^{(6)}$. Osmolarity of fecal water was measured (Osmomat 030-D, Gonotec, Berlin, Germany) and percentage of fecal wet weight was calculated ${ }^{(6)}$. Cytotoxicity of fecal water of physiologic osmolarity (300 mOsmol/L) was determined by potassium release of a human erythrocyte suspension after incubation with fecal water as previously described $^{(21)}$ and validated previously with intestinal epithelial cells ${ }^{(22)}$. Cytotoxicity was calculated and expressed as a percentage of maximal lysis.

\section{Analysis of enterobacteria and lactobacilli in fresh fecal samples}

Before infusion of TNBS or saline, fresh fecal samples were collected directly from the 
anus of the animals and analyzed for the number of enterobacteria and lactobacilli. Fecal enterobacteria were quantified by plating 10 -fold dilutions in saline on Levine EMB Agar (Difco Laboratories, Detroit, MI, USA) and incubating aerobically overnight at $37^{\circ} \mathrm{C}$. Lactobacilli were quantified by plating on Rogosa Agar (Oxoid, Basingstoke, UK) and incubating in anaerobic jars (MART Microbiology, Lichtenvoorde, The Netherlands) at $37^{\circ} \mathrm{C}$ for $2 \mathrm{~d}$.

\section{Histology}

The colonic tissue specimens were embedded in paraffin, and sections were stained with hematoxylin and eosin. Before analysis, slides were recoded to guarantee blind scoring and prevent observer bias. Colonic inflammation and deviations from normal mucosal architecture were evaluated using five different criteria, each scored from 0 to 3 , taking into account the area of the specimen involved. The criteria were leukocyte infiltration inmucosa, leukocyte infiltration in submucosa, goblet cell depletion, deviations from normal mucosal architecture, and discontinuity of the epithelial lining. The maximal possible score was 15 .

\section{Immunohistochemical staining for HSP-25 and HO-1}

For determination of HSP-25, sections were deparaffinized with xylene and subsequently hydrated with $100 \%, 96 \%, 70 \%, 50 \%$, and $0 \%$ ethanol in water. Thereafter, endogenous peroxidase activity was blocked for 15 min (Dako, Carpenteria, CA, USA). After every step, sections were washed with phosphate buffered saline. For antigen retrieval, slides were boiled twice for $10 \mathrm{~min}$ in $10 \mathrm{mmol} / \mathrm{L}$ of sodium citrate, $\mathrm{pH}$ 6.0. Next, non-specific binding was blocked using $0.8 \%$ gelatin in phosphate buffered saline for $30 \mathrm{~min}$. After blocking, slides were incubated for $1 \mathrm{~h}$ with a rabbit antibody against rat HSP-25 (1:200 in phosphate buffered saline; SPA-801, Stressgen, Victoria, BC, Canada), followed by 1-h incubation with horseradish peroxidase-labeled anti-rabbit antibody (Dako). Sections were stained for 5 min using diaminobenzidine solution (Dako) and counterstained with hematoxylin. After rehydration and mounting, slides were evaluated for epithelial HSP-25 expression using scores from 0 to 2 , taking into account the area of the specimen involved. HO-1 staining was performed as described by Dijkstra et al. ${ }^{(23)}$, and epithelial HO-1 expressionwas scored in the same way as HSP-25. Before analysis, slides were recoded to guarantee blind scoring and prevent observer bias.

\section{Analysis of $\alpha$-1-acid glycoprotein in serum}

The $\alpha$-1-acid glycoprotein (AGP) was measured in serum using an enzymelinked immunosorbent assay kit (Life Diagnostics, Inc., West Chester, PA, USA) according to the manufacturer's instructions.

\section{Statistical analysis}

Results are expressed as mean standard error of the mean or median $25 \%$ percentile, depending on the normality of distribution as indicated. The main predefined comparison of interest was a heme versus a control diet for TNBS-treated and shamtreated animals. Normally distributed data were analyzed using Student's t test, whereas 
non-normally distributed data were analyzed by Mann-Whitney U test (all two-sided). Differences were considered statistically significant at $\mathrm{P}<0.05$. The body weight data were analyzed by two-way analysis of variance with repeated measurements. Statistical analyses were conducted with GraphPad Prism 5.01 (GraphPad Software, Inc., La Jolla, CA, USA).

\section{RESULTS}

\section{Diarrhea and luminal cytotoxicity}

To test whether the irritating effect of dietary heme was indeed present, diarrhea and luminal cytotoxicity were determined. Relative fecal wet weight, which was measured to quantify diarrhea, was significantly higher in sham-treated heme-fed rats compared with rats fed the control diet, as found previously $(\mathrm{P}<0.05 \text {; Table } 2)^{(6)}$. After TNBS infusion, all rats developed diarrhea. Nevertheless, rats on the heme diet had significantly higher fecal wet weight levels compared with rats fed the control diet $(\mathrm{P}<0.05$; Table 2). In addition, the cytotoxicity assay was performed with fecal water of sham-treated rats. In line with previous studies ${ }^{(6)}$, dietary heme substantially increased luminal cytotoxicity compared with control rats $(99.8 \pm 0.2 \%$ versus $23.0 \pm 3.2 \%$, respectively, $\mathrm{P}<0.05)$.

\section{Colonic mRNA and protein expression of HSPs}

Inducible HSPs are produced in response to stress ${ }^{(8,9)}$. Therefore, we determined whether the irritating effect of heme leads to upregulation of inducible HSPs as hypothesized. Indeed, HSP-25 mRNA expression in colonic mucosa was increased 4.7fold in sham-treated heme-fed rats compared with rats on the control diet $(\mathrm{P}<0.05$; Figure 1A). In addition, heme significantly induced HO-1 (also known as HSP-32) mRNA expression 3.6-fold in colonic mucosa of sham-treated animals compared with control

Table 2: Effect of dietary heme on colonic parameters and fecal wet weight after colonic infusion of TNBS or sham treatment

\begin{tabular}{|c|c|c|c|c|c|}
\hline & \multicolumn{4}{|l|}{ Colon } & \multirow{2}{*}{$\begin{array}{c}\text { Fecal wet } \\
\text { weight (\%) }\end{array}$} \\
\hline & Weight $(g)$ & Length $(\mathrm{cm})$ & $\begin{array}{c}\text { Macroscopic } \\
\text { score }\end{array}$ & Histologic score & \\
\hline \multicolumn{6}{|c|}{ Sham treatment } \\
\hline Control & $0.5 \pm 0.03$ & $14.6 \pm 0.2$ & $0.8 \pm 0.2$ & $0.5 \pm 0.2$ & $64.8 \pm 2.3$ \\
\hline Heme & $0.5 \pm 0.03$ & $13.8 \pm 0.3^{*}$ & $0.2 \pm 0.2^{*}$ & $2.5 \pm 0.6^{*}$ & $87.2 \pm 0.6^{*}$ \\
\hline \multicolumn{6}{|c|}{ TNBS treatment } \\
\hline Control & $1.7 \pm 0.2$ & $12.3 \pm 0.4$ & $3.5 \pm 0.7$ & $10.3 \pm 1.0$ & $81.0 \pm 1.3$ \\
\hline Heme & $1.9 \pm 0.3$ & $10.8 \pm 0.3^{*}$ & $4.0 \pm 0.7$ & $12.5 \pm 1.2$ & $87.9 \pm 0.3^{*}$ \\
\hline
\end{tabular}

Results are expressed as mean \pm SEM. *Denotes that the heme group is significantly different from the control group within the same treatment group $(P<0.05)$. TNBS, trinitrobenzene sulfonic acid. 
rats $(\mathrm{P}<0.05$; Figure 1A). HSP-70-1 mRNA expression in sham-treated rats was not significantly influenced by heme (Figure 1A). Because TNBS is also a stressor for the colon, expression of HSPs was further increased in TNBS-treated rats (data not shown). It should be noted that we were interested in HSP expression in colonic mucosa before TNBS infusion and the effect of this HSP expression on the response to TNBS infusion. It was not in the scope of our research to study the simultaneous effect of diet and TNBS on HSP induction. Concordant with mRNA levels, HSP-25 and HO-1 protein expression was significantly increased in sham-treated heme-fed rats compared with rats on the control diet $(\mathrm{P}<0.05$; Figures $1 \mathrm{~B}$ and 2$)$.

\section{Enterobacteria and lactobacilli}

As described by Kojima et al. ${ }^{(24)}$, lipopolysaccharide, amajor component of gramnegative bacteria, may play a role in the induction of HSPs. To relate their finding to the present study, gram-negative enterobacteria and gram-positive lactobacilli were measured in fresh fecal samples. In this study, dietary heme increased the number of fecal enterobacteria about 10 -fold compared with the control group, whereas lactobacilli numbers were about 10 -fold lower in heme-fed animals $(\mathrm{P}<0.05$; Figure 3$)$.

\section{Mucosal inflammation markers}

Because we observed that heme was an intestinal stressor, which was associated with HSP induction, we were interested in the subsequent effect of dietary heme on colonic inflammation markers. Therefore, we measured MPO and IL-1 $\beta$ in mucosa. MPO concentrations in colonic mucosa were almost undetectable in sham-treated rats
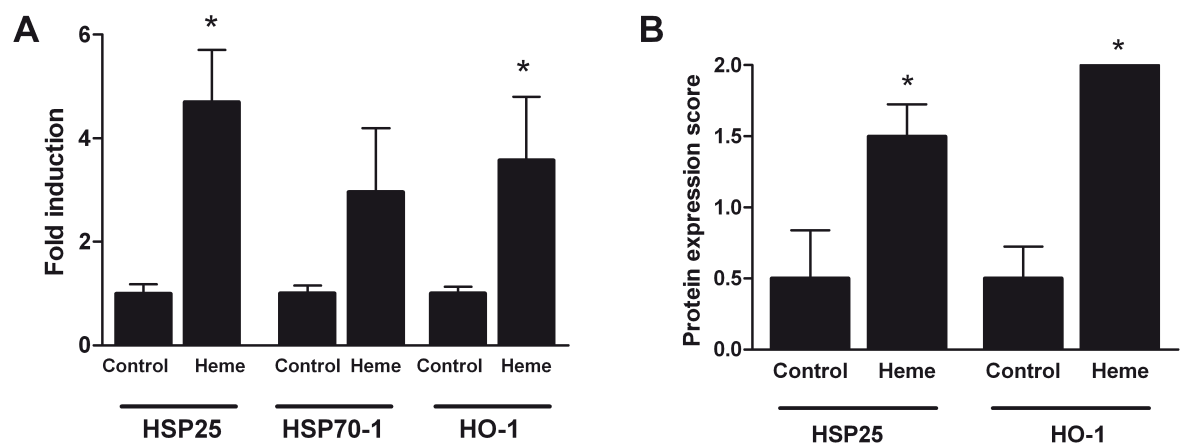

Figure 1: Effect of dietary heme on HSP expression in colonic mucosa of sham-treated rats. (A) Effect of heme on HSP-25, HSP-70-1, and HO-1 mRNA expression as determined by quantitative real-time polymerase chain reaction. The mean expression level of the control group was set at 1. (B) Effect of heme on HSP-25 and HO-1 protein expression as determined by scoring of histologic slides for epithelial HSP-25 and HO-1. Slides were scored from 0 to 2 depending on the extent of brown coloring due to HSP-25 and HO-1 expression. Results are expressed as mean \pm SEM. Messenger RNA levels of HSP-25 and HO-1 were significantly upregulated by dietary heme in sham-treated rats, and dietary heme significantly increased $\mathrm{HSP}-25$ and $\mathrm{HO}-1$ protein expression $\left({ }^{*} \mathrm{P}<0.05\right)$. $\mathrm{HO}$ 1, heme-oxygenase-1; HSP, heat-shock protein. 
(data not shown), whereas very high levels were measured after TNBS infusion. The heme diet lowered the TNBS-induced increase in colonic MPO $(\mathrm{P}<0.05$; Figure 4$)$. IL- $1 \beta$ levels in colonic mucosa were also increased due to TNBS treatment. In line with the MPO results, IL-1 $\beta$ protein decreased 3.2-fold in TNBS-treated rats on the heme diet compared with rats fed the control diet $(\mathrm{P}<0.05$; Figure 4$)$.

\section{Macroscopic and histologic changes of the colon}

Because MPO and IL-1 $\beta$ are just two proinflammatory markers, we were interested in whether these results could be translated into overall macroscopic and histologic effects of dietary heme. Colon weight, the semiquantitative macroscopic score of colon appearance, and the histologic score were clearly increased in all TNBS-treated rats. Colon length was shorter after TNBS treatment in control and heme-fed rats, a typical feature of intestinal inflammation. Interestingly, the colons of heme-fed rats were shorter than those fed the control diet for sham-treated and TNBS-treated animals $(\mathrm{P}<0.05$; Table 2). In the sham-treated heme-fed rats, the macroscopic score was significantly lower, whereas the histologic score was significantly higher compared with control rats $(\mathrm{P}<0.05$; Table 2$)$. Note that these latter scores are primarily intended to observe effects on inflammation. All sham-treated rats are taken as a non-colitic reference, and

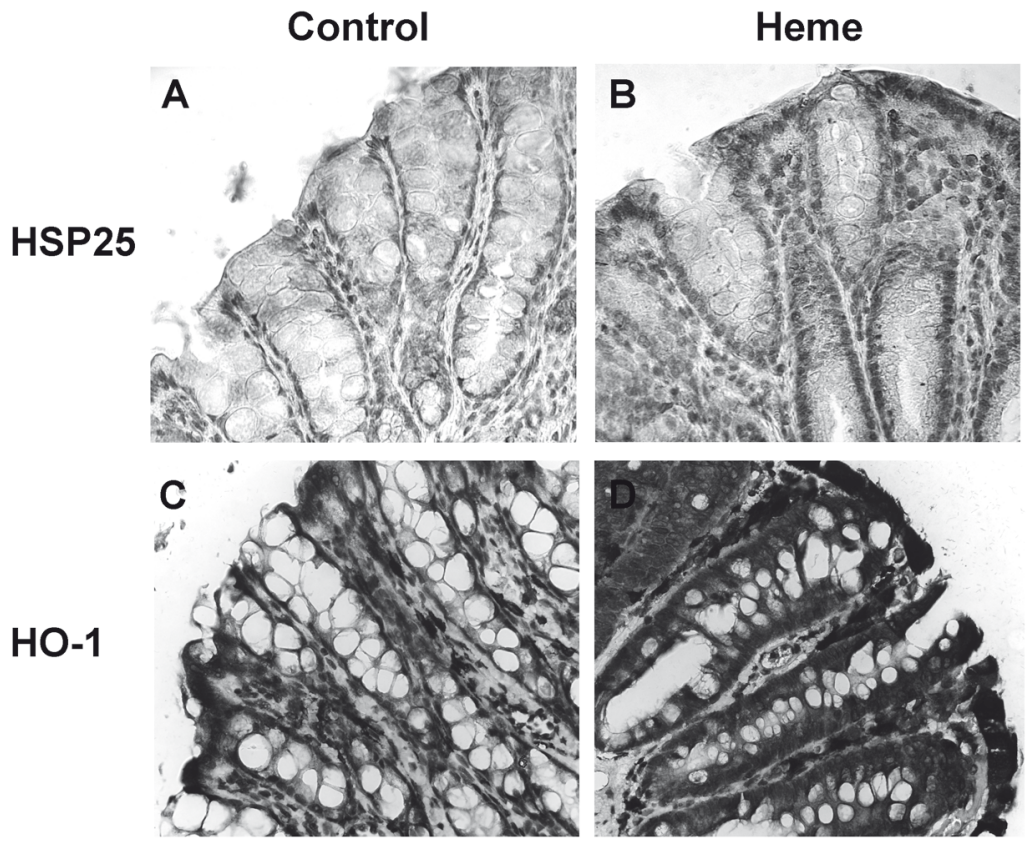

Figure 2: Immunohistochemical staining for HSP-25 and HO-1 in the colon. Images (magnification 40x) are representative sections taken from sham-treated rats fed the control diet $(A, C)$ or heme diet $(B, D)$ and stained for HSP-25 (A, B) or HO-1 (C, D). HO-1, heme-oxygenase-1; HSP-25, heat-shock protein-25. 
therefore dietary effects on these inflammatory parameters should be interpreted in light of this.

\section{General systemic health}

Because of the dichotomous effects of dietary heme, i.e., a lowering of the inflammatory markers MPO and IL-1 $\beta$, with no effect on histology but causing shortening of the colon, we were interested in the influence of heme on general systemic health during intestinal inflammation. Before saline or TNBS infusion, daily food intake of rats on the control diet $(20.7 \pm 0.4 \mathrm{~g} / \mathrm{d})$ was slightly higher than that of the rats on the heme diet $(19.0 \pm 0.3 \mathrm{~g} / \mathrm{d}, \mathrm{P}<0.05)$. Food consumption of all animals decreased sharply after TNBS administration. Nevertheless, food intake of animals on the control diet (5.3 $\pm 0.9 \mathrm{~g} / \mathrm{d})$ was significantly higher than food intake of rats fed the heme-supplemented diet (3.2 \pm $0.9 \mathrm{~g} / \mathrm{d}, \mathrm{P}<0.05)$ during the first $2 \mathrm{~d}$ after TNBS infusion. One week after TNBS infusion, food intake of heme-fed rats $(12.8 \pm 0.7 \mathrm{~g} / \mathrm{d})$ was still significantly lower compared with rats fed the control diet $(17.4 \pm 1.9 \mathrm{~g} / \mathrm{d}, \mathrm{P}<0.05)$. Just before saline or TNBS infusion, animals fed the control diet had a significantly higher body weight ( $349 \pm 4 \mathrm{~g}$ ) compared with heme-fed rats $(335 \pm 3 \mathrm{~g}$, $\mathrm{P}<0.05)$. During the first $2 \mathrm{~d}$ after TNBS infusion, weight loss was observed in both groups (20-30 g in 2 d; Figure 5). Importantly, the effect of the different diets was not the same at each point of time. Body weight regain $1 \mathrm{wk}$ after TNBS infusion was significantly less in heme-fed rats compared with rats fed the control diet $(\mathrm{P}<0.05$; Figure 5), indicating impaired recovery after the TNBS challenge. The acute-phase protein AGP was measured in serum as a marker of systemic stress in rats $^{(25)}$. In line with the colon length, body weight, and food intake results, heme-

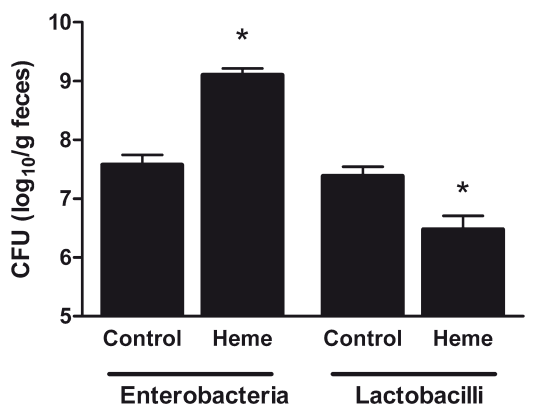

Figure 3: Effect of dietary heme on the number of enterobacteria and lactobacilli in fresh fecal samples of rats before infusion of saline. Results are expressed as mean \pm SEM. Dietary heme significantly increased the number of enterobacteria and significantly decreased the number of lactobacilli $\left({ }^{*} P<0.05\right)$. CFU, colony-forming unit.

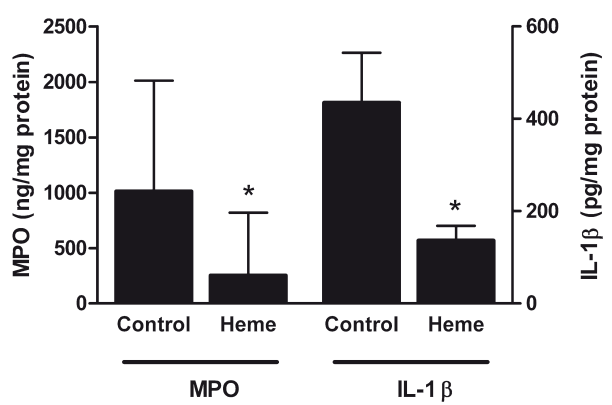

Figure 4: Effect of dietary heme on MPO and $I L-1 \beta$ concentrations in colonic mucosa of trinitrobenzene sulfonic acid-infused rats. MPO results are expressed as median (25\% percentile), and $I L-1 \beta$ results are expressed as mean \pm SEM. The MPO and $I L-1 \beta$ concentrations in the heme group are significantly lower than the MPO and IL-1 $\beta$ concentrations in the control group $\left({ }^{*} P<0.05\right)$. IL-1 $\beta$, interleukin-1 $\beta ; M P O$, myeloperoxidase. 
fed rats had higher serum AGP levels than control rats $(\mathrm{P}<0.05$; Figure $6 \mathrm{~A})$ after TNBS treatment. Therefore, overall systemic health during colitis was clearly worse in hemefed animals compared with control animals.

\section{Mucosal repair: Somatostatin}

Because the decreased body weight regain in heme-fed colitic rats probably indicates impaired recovery due to heme after the TNBS challenge, we were interested to find out what might be responsible to obtain a lead for further investigation. Therefore, we measured gene expression of some candidate genes involved in mucosal repair ${ }^{(26)}$. Heme supplementation led to a significant 3.0-fold downregulation of somatostatin gene expression in sham-treated rats $(\mathrm{P}<0.05$; Figure 6B). Importantly, somatostatin expression did not decrease further with TNBS infusion in the rats supplemented with heme, whereas it was 5.3-fold lower in control rats after infusion with TNBS compared with their sham-treated counterparts fed the control diet $(\mathrm{P}<0.05$; Figure 6B).

\section{DISCUSSION}

In the present study we investigated how dietary heme modulates the severity of colitis. We showed that dietary heme-induced luminal stress aggravated colonic mucosal inflammation in rats, despite an increase in colonic HSP expression. Although HSP induction was associated with decreased protein levels of the inflammatory markers MPO and IL-1 $\beta$, dietary heme overall adversely affected colitis development. Colon length, body weight regain, food intake, serum AGP concentration, and somatostatin gene expression were adversely affected in the heme-fed colitic rats. Previous studies in the field of colorectal cancer have shown that dietary heme, mimicking red meat,

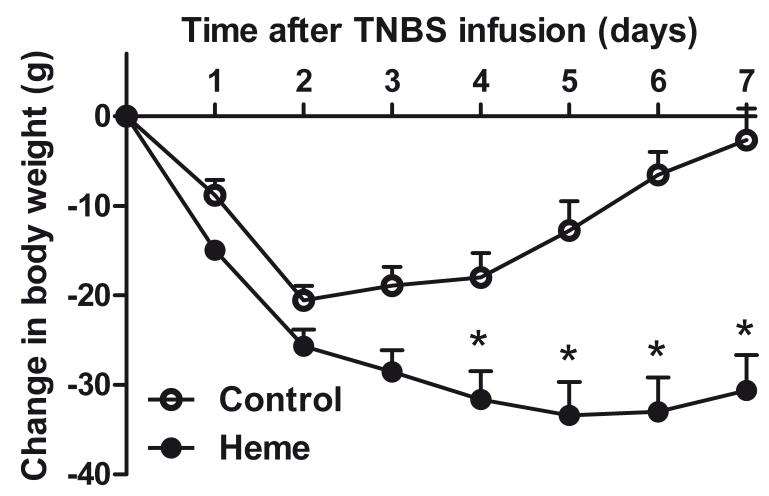

Figure 5: Effect of dietary heme on body weight of rats infused with TNBS. Results are expressed as mean change \pm SEM in body weight compared with body weight just before infusion of TNBS. The effect of the different diets is not the same at each point of time. From day 4 the control rats start gaining weight, whereas the heme-fed animals showed an impaired recovery $\left({ }^{*} P<0.05\right)$. TNBS, trinitrobenzene sulfonic acid. 
creates a cytotoxic environment in the gut lumen, causing colonic epithelial damage and subsequent compensatory hyperproliferation ${ }^{(6)}$. Indeed, dietary heme supplementation increased cytotoxicity of intestinal contents in the present study. The irritating effect of heme was accompanied by the upregulation of HSPs. The concept of HSP induction being protective against subsequent inflammation is currently being extensively investigated $^{(7,10-15)}$. However, the irritating influence of heme, which is continuously present in the colon, possibly over-ruled potential beneficial HSP induction. Moreover, it seems that heme impaired recovery after TNBS infusion, as observed by the compromised body weight regain, thus aggravating colitis development. In addition, we speculate that somatostatin plays a role in the heme-induced disordered mucosal repair $^{(26)}$.

Inducible HSPs, particularly HSP-25, HSP-70, and HO-1, are produced in response to several kinds of stress and serve to stabilize other proteins and preserve their function ${ }^{(8,9)}$. Indeed, HSP-25 was upregulated in colonic mucosa of sham-treated rats on the heme diet. The effect of this protein in colonic epithelial cells has been extensively examined by Kojima et al. ${ }^{(24)}$. Their in vitro experiments showed that specific induction of HSP-25 by lipopolysaccharide from gram-negative bacteria conferred protection against the oxidant monochloramine. In another cell study it was shown that colonic bacteria also contribute to specific HSP-25 expression by the production of short-chain fatty acids, which then protected against oxidant-induced stress. In vivo, increasing fermentable fiber in the diet of rats resulted in upregulation of colonic HSP-25(27). Comparable to the results of Kojima et al., dietary heme stimulated the growth of gramnegative enterobacteria about 10 -fold in this study, whereas gram-positive lactobacilli
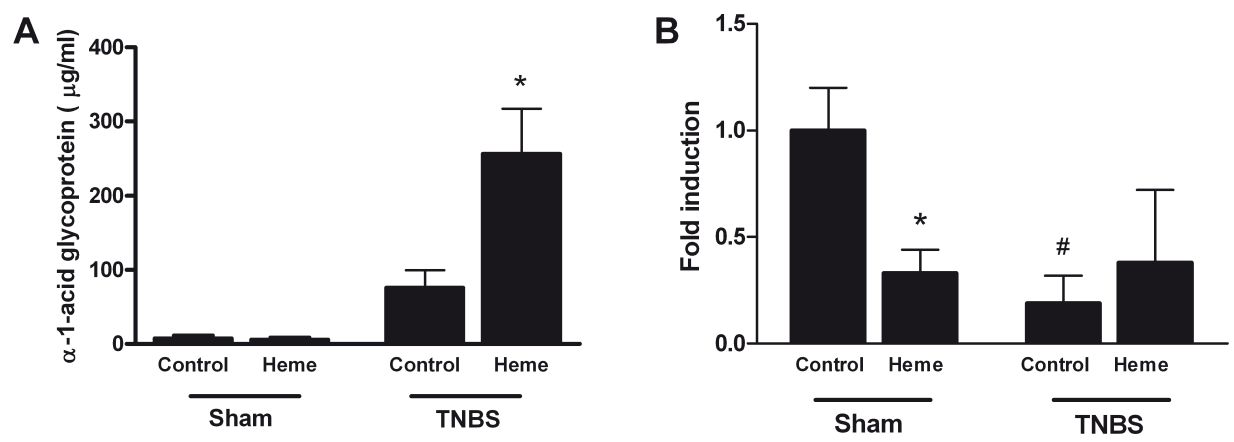

Figure 6: Effect of dietary heme on serum a-1-acid glycoprotein levels and colonic mucosal somatostatin mRNA expression in rats after colonic infusion of TNBS or sham-treatment. (A) Effect of heme on a-1-acid glycoprotein levels in serum as determined by enzyme-linked immunosorbent assay. (B) Effect of heme on somatostatin mRNA expression as determined by quantitative real-time polymerase chain reaction. The mean expression level of the control group was set at 1. Results are expressed as mean \pm SEM. Serum a-1-acid glycoprotein levels were significantly increased by dietary heme in TNBS-treated rats $\left({ }^{*} P<0.05\right)$. Messenger RNA levels of somatostatin were significantly downregulated by dietary heme in sham-treated rats $\left({ }^{*} P<0.05\right)$. Also, after TNBS infusion somatostatin mRNA levels were downregulated in the control group $\left({ }^{\sharp} P<0.05\right)$, but not in the heme-fed rats. TNBS, trinitrobenzene sulfonic acid. 
numbers were about 10 -fold lower in heme-fed animals. Likewise, induction of epithelial stress by heme, possibly mediated by lipopolysaccharide from gram-negative bacteria, might have triggered colonic HSP-25 expression. Importantly, subsequent mucosal inflammation was not decreased in the present study. Because gut microbiota play an important role in the pathogenesis of colitis ${ }^{(28,29)}$, the effect of dietary heme on the balance of gram-negative and gram-positive bacteria is of particular interest and will be the subject of further investigation.

We also investigated the induction of HO-1, also known as HSP-32. HO-1 was upregulated in the heme group compared with rats fed the control diet. Heme is the substrate and primary inducer of HO-1. HO-1 liberates iron from the porphyrin structure and catabolizes heme into biliverdin and carbon monoxide. Protective effects of HO-1 are ascribed to these reaction products ${ }^{(7)}$. Expression of HSP-70-1 was not regulated by dietary heme in the present study. This is in line with the results of Kojima et al. ${ }^{(24)}$ and Ren et al. ${ }^{(27)}$, where they also observed only HSP-25 induction and no increase in HSP-70 after exposure of cells to lipopolysaccharide or short-chain fatty acids. However, various forms of stress might induce different (sets of) HSPs, potentially leading to cytoprotection, although mediated by different mechanisms. Interestingly, induction of HSP-70 alone ${ }^{(11-12,14)}$ or HSP-25 and HSP-70 ${ }^{(13)}$ has been suggested to play a role in amelioration of colitis. In addition, HO-1 is known for its anti-inflammatory actions and has been suggested as a target for treatment of $\operatorname{IBD}^{(7,10)}$. In contrast to these studies, we did not find HSP-associated protective effects on intestinal inflammation. Alternative ways to induce HSPs locally and ameliorate intestinal inflammatory processes without the unwanted systemic side effects might still be interesting to explore, e.g., by using less toxic stimuli. However, the results of this study do not support the concept of inducing HSPs as protective in subsequent intestinal inflammation.

The discrepancy between the effect of heme on local colonic HSPs and inflammatory markers, on the one hand, and systemic health, as determined by body weight gain, food intake, colon length, and serum AGP levels, on the other hand, is interesting. This might be explained by the fact that the irritating influence of heme is continuously present in the colon and not just a single "hit," meaning that dietary heme can constantly modulate the severity of colitis. Moreover, these observations further underline the importance of addressing systemic effects in colitis research rather than focusing only on colon results. The constant presence of heme in the colon might have caused the decreased body weight regain of the heme-fed colitic rats, which probably results from impaired recovery after the TNBS challenge. To investigate what played a role in the decreased mucosal repair due to heme, some candidate genes involved in tissue regeneration were examined $^{(26)}$. Somatostatin has been shown to inhibit intestinal regeneration ${ }^{(30)}$. In line with this, dietary heme led to downregulation of somatostatin expression in sham-treated rats, so repair was induced after the heme stress. However, somatostatin expression did not decrease further with TNBS infusion in the heme-fed rats, whereas it decreased in TNBS-infused rats on the control diet compared with their sham-treated counterparts on the control diet. These results suggest that the heme-fed rats were not able to compensate for the second intestinal stressor. Therefore, the lack of regulation of somatostatin by heme after TNBS infusion might have played a role in the impaired 
intestinal repair due to heme. However, further research is necessary to support these findings.

The dietary heme concentration used in this study corresponds to a beef intake of 500 $\mathrm{g} / \mathrm{d}$ in humans ${ }^{(31)}$, which is relatively high. However, similar effects of lower doses of heme on luminal cytotoxicity have been reported ${ }^{(32)}$. Our aim was to show proof of concept for the effect of heme on colitis development, and it is common in nutritional studies to use a rather high but not unrealistic concentration of the nutrient of interest. We do not want to promote a high intake of dietary heme (or red meat) in humans. This is line with epidemiology, demonstrating that high meat intake, in particular red meat, increases the risk of relapse for patients with colitis ${ }^{(4)}$. Prior research in colon cancer also warrants this restraint. Interestingly, other results from this field have shown that dietary calcium and chlorophyll can counteract the heme-induced intestinal stress $^{(31,33)}$. Elaborating on the dietary modulation of intestinal inflammation, it might be interesting to investigate these counteractive effects of calcium or chlorophyll on heme with respect to IBD.

\section{CoNCLUSION}

The present study shows that dietary heme, mimicking red meat, aggravated the severity of colitis. Importantly, intestinal heme stress caused a potentially beneficial HSP induction, but this did not decrease subsequent TNBS-induced mucosal inflammation in rats. Adverse systemic effects on colitis development were observed. We hypothesize that the irritating heme stress, being continuously present in the colon, over-ruled the potentially favorable HSP induction and impaired recovery. The present results emphasize the potential of nutrition to modulate mucosal inflammation. Moreover, this study implies that a diet high in red meat might be a risk factor for the development of IBD. However, further studies are necessary to confirm these findings.

\section{Acknowledgments}

The authors thank the biotechnicians at the Small Animal Centre of Wageningen University (Wageningen, The Netherlands) and scientific assistant Lisette Bok (University Medical Center Groningen) for their expert assistance and Robert-Jan Brummer (Top Institute Food and Nutrition, Wageningen, The Netherlands) for stimulating discussions.

\section{REFERENCES}

1. Fiocchi C. Inflammatory bowel disease: etiology and pathogenesis. Gastroenterology 1998;115:182205.

2. Sartor RB. Mechanisms of disease: pathogenesis of Crohn's disease and ulcerative colitis. Nat Clin Pract Gastroenterol Hepatol 2006;3:390-407.

3. Ling SC, Griffiths AM. Nutrition in inflammatory bowel disease. Curr Opin Clin Nutr Metab Care 2000;3:339-44.

4. Jowett SL, Seal CJ, Pearce MS, Phillips E, Gregory W, Barton JR, et al. Influence of dietary factors on the clinical course of ulcerative colitis: a prospective cohort study. Gut 2004;53:1479-84. 
5. Chao A, Thun MJ, Connell CJ, McCullough ML, Jacobs EJ, Flanders WD, et al. Meat consumption and risk of colorectal cancer. JAMA 2005;293:172-82.

6. Sesink AL, Termont DS, Kleibeuker JH, Van der Meer R. Red meat and colon cancer: the cytotoxic and hyperproliferative effects of dietary heme. Cancer Res 1999;59:5704-9.

7. Otterbein LE, SoaresMP, Yamashita K, Bach FH.Heme oxygenase-1: unleashing the protective properties of heme. Trends Immunol 2003;24:449-55.

8. Otaka M, Odashima M, Watanabe S. Role of heat shock proteins (molecular chaperones) in intestinal mucosal protection. Biochem Biophys Res Commun 2006;348:1-5.

9. Petrof EO, Ciancio MJ, Chang EB. Role and regulation of intestinal epithelial heat shock proteins in health and disease. Chin J Dig Dis 2004;5:45-50.

10. Naito Y, Takagi T, Yoshikawa T. Heme oxygenase-1: a new therapeutic target for inflammatory bowel disease. Aliment Pharmacol Ther 2004;20(suppl 1):177-84.

11. Odashima M, Otaka M, Jin M, Wada I, Horikawa Y, Matsuhashi T, et al. Zinc l-carnosine protects colonic mucosal injury through induction of heat shock protein 72 and suppression of NF-kappaB activation. Life Sci 2006;79:2245-50.

12. Ohkawara T, Nishihira J, Takeda H, Katsurada T, Kato K, Yoshiki T, et al. Protective effect of geranylgeranylacetone on trinitrobenzene sulfonic acid-induced colitis in mice. Int J Mol Med 2006; 17:229-34.

13. Ohkawara T, Takeda H, Kato K, Miyashita K, Kato M, Iwanaga T, et al. Polaprezinc (N-(3-aminopropionyl)L-histidinato zinc) ameliorates dextran sulfate sodium-induced colitis in mice. Scand J Gastroenterol 2005;40:1321-7.

14. Otani S, Otaka M, Jin M, Okuyama A, Itoh S, Iwabuchi A, et al. Effect of preinduction of heat shock proteins on acetic acid-induced colitis in rats. Dig Dis Sci 1997;42:833-46.

15. Sikora A, Grzesiuk E. Heat shock response in gastrointestinal tract. J Physiol Pharmacol 2007;58(suppl 3):43-62.

16. Morris GP, Beck PL, Herridge MS, Depew WT, Szewczuk MR, Wallace JL. Hapten-induced model of chronic inflammation and ulceration in the rat colon. Gastroenterology 1989;96:795-803.

17. Elson CO, Sartor RB, Tennyson GS, Riddell RH. Experimental models of inflammatory bowel disease. Gastroenterology 1995;109:1344-67.

18. Reeves PG, Nielsen FH, Fahey GC Jr. AIN-93 purified diets for laboratory rodents: final report of the American Institute of Nutrition ad hoc writing committee on the reformulation of the AIN-76A rodent diet. J Nutr 1993;123:1939-51.

19. Briefel RR, Johnson CL. Secular trends in dietary intake in the United States. Annu Rev Nutr 2004;24:40131.

20. Wallace JL, Keenan CM. An orally active inhibitor of leukotriene synthesis accelerates healing in a rat model of colitis. Am J Physiol Gastrointest Liver Physiol 1990;258:G527-34.

21. Bovee-Oudenhoven I, Termont D, Dekker R, Van der Meer R. Calcium in milk and fermentation by yoghurt bacteria increase the resistance of rats to Salmonella infection. Gut 1996;38:59-65.

22. Lapre JA, Termont DS, Groen AK, Van der Meer R. Lytic effects of mixed micelles of fatty acids and bile acids. Am J Physiol Gastrointest Liver Physiol 1992;263:G333-7.

23. Dijkstra G, Blokzijl H, Bok L, Homan M, van Goor H, Faber KN, et al. Opposite effect of oxidative stress on inducible nitric oxide synthase and haem oxygenase-1 expression in intestinal inflammation: antiinflammatory effect of carbon monoxide. J Pathol 2004;204:296-303.

24. Kojima K, MuschMW, Ropeleski MJ, Boone DL, Ma A, Chang EB. Escherichia coli LPS induces heat shock protein 25 in intestinal epithelial cells through MAP kinase activation. Am J Physiol Gastrointest Liver Physiol 2004;286:G645-52.

25. Fournier T, Medjoubi NN, Porquet D. Alpha-1-acid glycoprotein. Biochim Biophys Acta 2000;1482:15771.

26. Moss SF, Wright NA. Molecular aspects of mucosal repair: a summary. Yale J Biol Med 1996;69:155-8.

27. Ren H, Musch MW, Kojima K, Boone D, Ma A, Chang EB. Short-chain fatty acids induce intestinal epithelial heat shock protein 25 expression in rats and IEC 18 cells. Gastroenterology 2001;121:631-9.

28. Sartor RB. Microbial influences in inflammatory bowel diseases. Gastroenterology 2008;134:577-94.

29. Rakoff-Nahoum S, Paglino J, Eslami-Varzaneh F, Edberg S, Medzhitov R. Recognition of commensal microflora by toll-like receptors is required for intestinal homeostasis. Cell 2004;118:229-41. 
Chapter 2

30. Thompson JS, Nguyen BL, Harty RF. Somatostatin analogue inhibits intestinal regeneration. Arch Surg 1993;128:385-9.

31. de Vogel J, Jonker-Termont DS, van Lieshout EM, Katan MB, van der Meer R. Green vegetables, red meat and colon cancer: chlorophyll prevents the cytotoxic and hyperproliferative effects of haem in rat colon. Carcinogenesis 2005;26:387-93.

32. Pierre F, Tache S, Petit CR, Van der Meer R, Corpet DE. Meat and cancer: haemoglobin and haemin in a low-calcium diet promote colorectal carcinogenesis at the aberrant crypt stage in rats. Carcinogenesis 2003;24:1683-90.

33. Sesink AL, Termont DS, Kleibeuker JH, Van der Meer R. Red meat and colon cancer: dietary haeminduced colonic cytotoxicity and epithelial hyperproliferation are inhibited by calcium. Carcinogenesis 2001;22:1653-9. 


\section{Supplemental calcium attenuates the}

colitis-related increase in diarrhea, intestinal permeability, and extracellular matrix breakdown in HLA-B27 transgenic rats

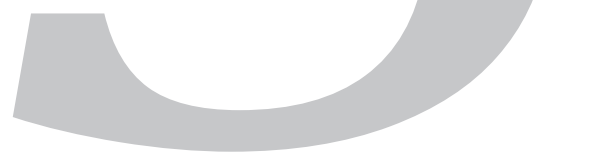

Marloes A. A. Schepens Arjan J. Schonewille Carolien Vink Evert M. van Schothorst Evelien Kramer Thijs Hendriks Robert-Jan Brummer Jaap Keijer Roelof van der Meer Ingeborg M. J. Bovee-Oudenhoven

The Journal of Nutrition. 2009 Aug; 139(8): 1525-1533 


\section{Abstract}

Objective: We have shown in several controlled rat and human infection studies that dietary calcium improves intestinal resistance and strengthens the mucosal barrier. Reinforcement of gut barrier function may alleviate inflammatory bowel disease (IBD). Therefore, we investigated the effect of supplemental calcium on spontaneous colitis development in an experimental rat model of IBD.

Methods: HLA-B27 transgenic rats were fed a purified high-fat diet containing either a low or high calcium concentration (30 and $120 \mathrm{mmol} \mathrm{CaHPO}_{4} / \mathrm{kg}$ diet, respectively) for almost $7 \mathrm{wk}$. Inert chromium EDTA (CrEDTA) was added to the diets to quantify intestinal permeability by measuring urinary CrEDTA excretion. Relative fecal wet weight was determined to quantify diarrhea. Colonic inflammation was determined histologically and by measuring mucosal interleukin (IL)-1 $\beta$. In addition, colonic mucosal gene expression of individual rats was analyzed using wholegenome microarrays.

Results: The calcium diet significantly inhibited the increase in intestinal permeability and diarrhea with time in HLA-B27 rats developing colitis compared with the control transgenic rats. Mucosal IL-1 $\beta$ levels were lower in calciumfed rats and histological colitis scores tended to be lower $(\mathrm{P}=0.08)$. Supplemental calcium prevented the colitisinduced increase in the expression of extracellular matrix remodeling genes (e.g. matrix metalloproteinases, procollagens, and fibronectin), which was confirmed by quantitative real-time PCR and gelatin zymography.

Conclusion: In conclusion, dietary calcium ameliorates several important aspects of colitis severity in HLA-B27 transgenic rats. Reduction of mucosal irritation by luminal components might be part of the mechanism. These results show promise for supplemental calcium as effective adjunct therapy for IBD.

Supplemental tables 1 and 2 and supplemental figure 1: see after references 


\section{INTRODUCTION}

Inflammatory bowel disease (IBD), which includes Crohn's disease and ulcerative colitis, is a chronic relapsing and remitting disorder characterized by intestinal inflammation. The exact etiology of IBD is still unknown, but the disease is considered multifactorial. Various determinants in a genetically susceptible individual are involved in the pathogenesis, namely the gutmucosal barrier, the immune system, and microbial factors ${ }^{(1)}$. Nutrition is of particular importance, because it can modulate these determinants. Therefore, dietary modulation of IBD can be an attractive option to prevent relapse and reduce disease symptoms ${ }^{(2)}$.

We have shown in several controlled studies that dietary calcium protects against intestinal infections with food-borne bacterial pathogens in both rats and humans ${ }^{(3-5)}$. Besides preventing intestinal bacterial translocation, calcium has cytoprotective effects, as shown in several studies in the field of colon carcinogenesis. By precipitating irritating bile acids and fatty acids, supplemental calcium reduces cytotoxicity of the fecal stream. This decrease in luminal cytotoxicity reduces damage to intestinal epithelial cells, which reinforces mucosal integrity ${ }^{(6,7)}$. Based on these generic protective effects of calcium on gut health, we hypothesized that dietary calcium also has beneficial effects on intestinal inflammation.

Despite some studies on calcium and bone health in IBD, the influence of calcium on intestinal inflammation has hardly been investigated. To our knowledge, up to now, 2 studies have reported on possible protective effects of dietary calcium on intestinal inflammation. However, Zhu et al. ${ }^{(8)}$ mainly focused on vitamin $D$ and did not examine in much detail the effect of calcium, while the results of Pele et al..$^{(9)}$ should be interpreted with caution, because a direct interaction between calcium and the chemical applied to induce colitis cannot be excluded. Furthermore, we aimed to investigate the potential beneficial effect of calcium more thoroughly by including intestinal permeability measurements and mucosal gene expression profiling in the present study.

The HLA-B27 transgenic rat is a well-characterized model of chronic intestinal inflammation. This model allows us to perform controlled nutritional interventions and detailed colonic analyses to determine nutrient efficacy. Rats overexpressing the human HLA-B27/ $\beta 2$ microglobulin gene spontaneously develop an inflammatory disease involving mainly the gastrointestinal tract and the colon in particular. Also in humans, the HLA-B27 gene is associated with inflammatory disorders, including IBD. HLA-B27expressing innate immune cells and the presence of enteric bacteria are essential in the development of intestinal inflammation in this model ${ }^{(10)}$. Although the exact responsible mechanism has not been characterized yet, it is hypothesized that microbial recognition through Toll-like receptors is heightened in these rats ${ }^{(11)}$. Therefore, as luminal stressors are important in the development of colitis in this rat model, calcium might exert protective effects by decreasing luminal cytotoxicity.

In the present study, we investigated the effect of dietary calcium on intestinal inflammation in HLA-B27 transgenic rats. Because of the above-mentioned protective effects of calcium on both intestinal infection and colon carcinogenesis, we hypothesized 
that dietary calcium also reduces the severity of colitis, as generic protective mechanisms in the gut lumen are likely involved. We expected that decreasing luminal cytotoxicity by calcium would strengthen the mucosal barrier, which is considered important for prevention of colitis.

\section{MATERIALS AND MethodS}

\section{Experimental design: rats and diets}

The experimental protocol was approved by the animal welfare committee of Wageningen University (The Netherlands). Female HLA-B27/ $\beta 2$-microglobulin transgenic rats on an inbred Fisher 344 background and their nontransgenic counterparts $(n=7)$ (Taconic Farms), 8-10 wk old and with a mean body weight of $128 \mathrm{~g}$ at the start of the experiment, were housed individually in metabolic cages. Unfortunately, 1 transgenic rat died at the start of the experiment while orbital blood was obtained. Therefore, 9 transgenic rats were included in the control group and 8 transgenic rats in the calcium group. Three nontransgenic rats were fed the control diet and 4 were fed the calcium diet. Rats were kept in a temperature- and humidity-controlled environment and in a 12-h-light/-dark cycle. Rats consumed a purified "humanized" Western diet ad libitum. The diets were low in calcium (30 mmol/kg diet; Sigma-Aldrich) and had a high-fat content to mimic the composition of aWestern human diet. The calcium diet contained $120 \mathrm{mmol}$ calcium/kg diet at the expense of glucose. Inert chromium EDTA (CrEDTA) was added to the diets to quantify intestinal permeability ${ }^{(12)}$. The exact composition of the diets is provided in Table 1. CrEDTA solution was prepared as described elsewhere and subsequently freeze-dried ${ }^{(13)}$. To check the complete formation and stability of the CrEDTA complex, the prepared CrEDTA solution was passed through a cation-exchange resin column (Chelex 100 Resin; Bio-Rad). No uncomplexed $\mathrm{Cr}^{3+}$ ions were present. Food intake was recorded daily and body weight twice every week. At the start of the experiment, orbital blood was obtained under isoflurane anesthesia for preparation of serum. In advance, time of manifestation of colitis could not be predicted. Therefore, intestinal permeability and diarrhea were monitored every 1 or 2 wk to determine the appropriate moment of section. Almost $7 \mathrm{wk}$ after the start of the experiment, when a clear increase in intestinal permeability and diarrhea was measurable in at least 1 dietary group (see Results), rats were killed by carbon dioxide inhalation and orbital blood was again obtained for preparation of serum. The colon was removed, washed in saline, and weight and length were measured. A 1-cm piece from the middle of the colon was cut out and stored in 10\% neutral buffered formalin for histology. The remaining colon parts were longitudinally excised. Subsequently, the mucosa was scraped off using a spatula and immediately frozen in liquid nitrogen until further processing and analyses.

\section{Fecal analyses}

All feces were collected for $3 \mathrm{~d}$ every $2 \mathrm{wk}$ and freezedried. Percentage fecal wet weight was determined to quantify diarrhea. Fecal water was prepared as described 
Table 1: Composition of the experimental diets

\begin{tabular}{lcc}
\hline Ingredients & Control diet & Calcium diet \\
\hline & & $\mathrm{g} / \mathrm{kg}$ \\
Acid casein & 200 & 200 \\
Corn starch & 326 & 326 \\
Glucose & 172 & 156 \\
Palm oil & 160 & 160 \\
Corn oil & 40 & 40 \\
Cellulose & 50 & 50 \\
CrEDTA & 2 & 2 \\
CaHPO $2{ }_{4} 2 \mathrm{H}_{2} \mathrm{O}$ & 5.16 & 20.65 \\
Vitamin mix & 10 & 10 \\
Mineral mix & 35 & 35 \\
\hline
\end{tabular}

Vitamins and minerals were added to both diets according to AIN93(44) with the following adjustments: calcium was omitted from the mineral mix, because it was added separately to both diets; potassium citrate was added instead of potassium phosphate; chromium potassium sulfate was omitted, because CrEDTA was added to the diets; and choline chloride was added instead of choline bitartrate. Besides acid casein, no additional amino acids were added.

earlier $^{(14)}$. Cytotoxicity of fecal water of physiological osmolarity (300 m0smol/L) was determined by potassium release of a human erythrocyte suspension after incubation with fecal water, as previously described ${ }^{(15)}$, and validated earlier with intestinal epithelial cells ${ }^{(16)}$. Cytotoxicity was calculated and expressed as a percentage of maximal lysis. For measurement of calcium in fecal water, fecal water was acidified with $50 \mathrm{~g} / \mathrm{L}$ trichloroacetic acid, centrifuged, diluted with $0.5 \mathrm{~g} / \mathrm{L} \mathrm{CsCl}$, and analyzed by inductively coupled plasma-atomic emission spectrophotometry (Varian).

\section{Measurement of intestinal permeability}

Total 24-h urine samples were collected on 1 day every week. For CrEDTA measurement, urine was acidified with $50 \mathrm{~g} / \mathrm{L}$ trichloroacetic acid, centrifuged at 14,000 x g; 2 min and diluted with $0.5 \mathrm{~g} / \mathrm{L} \mathrm{CsCl}$. Then, chromium was analyzed by inductively coupled plasmaatomic emission spectrophotometry. Antilipopolysaccharide (LPS) antibodies in serum, induced upon leakage of LPS through the intestinal mucosa, were determined using an ELISA kit (EndoCab; Hycult biotechnology; detection limit: 125 arbitrary units/L) using goat anti-rat antibodies as tracer (1:20,000; Sigma-Aldrich).

\section{Colonic histology}

Colonic tissue specimens were embedded in paraffin and slices were stained with hematoxylin and eosin. Before analysis, slides were recoded to guarantee scoring was conducted without knowledge of the treatment groups. A validated histological scoring 
system for inflammation was used. Scores ranged from 0 to 4 based on criteria such as inflammatory cell infiltration, depletion of goblet cells, mucosa thickening, and destruction of mucosal architecture ${ }^{(17)}$.

\section{Myeloperoxidase and interleukin-1 $\beta$ analysis in colonic mucosa}

Frozen colonic mucosal scrapings were manually crushed in liquid nitrogen using a precooled mortar and pestle. After homogenization, part of the powder was used for RNA isolation (see below) and the remaining portion was suspended in a solution containing $200 \mathrm{mmol} / \mathrm{L}$ sucrose, $20 \mathrm{mmol} / \mathrm{L}$ Tris, $1 \mathrm{mmol} / \mathrm{L}$ dithiothreitol, and protease inhibitors before protein analyses. Myeloperoxidase (MPO) (Hycult biotechnology) and interleukin (IL)-1 $\beta$ (Biosource) were determined using ELISA kits (detection limits were $1.0 \mu \mathrm{g} / \mathrm{L}$ and $3.0 \mathrm{ng} / \mathrm{L}$, respectively) according to the manufacturers' instructions. IL-1 $\beta$ was measured in preference to tumor necrosis factor- $\alpha$ as an important proinflammatory marker, because it has been described that tumor necrosis factor- $\alpha$ levels are much less in tissue of HLA-B27 transgenic rats ${ }^{(18)}$. Total protein content of the scrapings was measured spectrophotometrically using the BCA protein assay kit (Uptima) with bovine serum albumin (Sigma-Aldrich) as standard.

\section{Colonic mucosal RNA isolation}

Total RNA was isolated from frozen homogenized colonic scrapings using TRIzol reagent (Invitrogen) according to the manufacturer's protocol. RNA was purified with RNeasy columns (Qiagen) and purity and concentration were quantified using the Nanodrop ND-1000 (Isogen Life Science). $A_{260}: A_{280}$ ratios were all between 1.83 and 2.16, indicating RNA of high purity. Additionally, we checked RNA quality using the Experion (Bio-Rad), showing absence of RNA degradation.

\section{Microarray hybridization of mucosal samples}

Amplification, labeling, and microarray hybridization of colonic mucosal samples of individual transgenic rats were performed following the Agilent procedures for whole-genome microarray technology (Agilent Technologies) unless stated otherwise. Briefly, $1 \mu \mathrm{g}$ total RNA from each rat was reverse transcribed. Each cDNA sample was split into 2 fractions that were subsequently used for linear RNA amplification and labeled with Cy5 and Cy3, respectively, using one-half the amounts indicated by the manufacturer. The labeled cRNA samples were purified using RNeasy columns (Qiagen). We measured dye incorporation and cRNA concentration using the Nanodrop ND-1000 spectrophotometer. Yield of each individual sample was $>825 \mathrm{ng}$ and specific activity $>8.0$ pmol Cy3 or Cy5/ $\mu$ g cRNA. Equal amounts of Cy3 cRNA of all rats were pooled to serve as standard reference pool. After fragmentation, samples containing Cy5-labeled cRNA of individual rats and Cy3-labeled pool cRNA were hybridized on $4 \mathrm{x} 44 \mathrm{~K}$ rat whole-genome Agilent arrays (G4131F). After washing, the arrays were scanned with an Agilent G2565B microarray scanner on the basis of integrating both the low- and high- intensity scans per array to obtain a larger linear range for spot fluorescence quantification. 


\section{Data analysis of microarray experiment}

Signal intensities for each spot were quantified using Feature Extraction 9.5 (Agilent Technologies). Median density values and background values of each spot were extracted for both the experimental samples (Cy5) and the reference samples (Cy3). Quality checks based on raw data were performed for each microarray using both the LimmaGUI package in R from Bioconductor ${ }^{(19)}$ and Microsoft Excel. Data were exported into GeneMaths XT 1.60 (Applied Maths) for analysis. We discarded spots with a mean intensity, over all arrays, of Cy $5<2$-fold above the mean background intensity. Then, the Cy5 intensities were normalized as described before ${ }^{(20)}$. Genes that significantly changed (Student's t test) in response to calcium were selected for pathway analysis. Gene expression results are reported as fold-change of calcium-fed transgenic rats compared with control transgenic rats. Fold-changes $<1$ are reported as their negative inverse. P-values were only used to prioritize data based on highest significance for further analysis. Pathway analyses were performed using both MetaCore (GeneGo) and Ermine ${ }^{(21)}$. Microarray data are publicly accessible in the National Center for Biotechnology Information's Gene Expression Omnibus with accession number GSE11022.

\section{Quantitative real-time PCR on mucosal samples}

Total RNA $(1 \mu \mathrm{g})$ of individual samples from both transgenic and nontransgenic rats was used for cDNA synthesis using the iScript cDNA synthesis kit of Bio-Rad. Real-time reactions were performed using the MyIQ real-time PCR detection system (Bio-Rad), as described previously ${ }^{(22)}$, with primers designed using Beacon designer 7.00 (Premier Biosoft International) (Supplemental Table 1).

Data were normalized to the geometrical mean of 3 reference genes using GeNorm ${ }^{(23)}$ : aldolase, ADP-ribosylation factor 1 , and $\beta$-actin. These genes showed no regulation due to the calcium intervention based on the array data. A standard curve for all genes was generated using serial dilutions of a pooled sample containing cDNA from all reactions, with the following exception: for detection of matrix metalloproteinase (MMP), a pooled sample of cDNA of only control transgenic rats (where MMP were most abundantly expressed) was used. Individual sample mRNA levels were determined in duplicate from the appropriate standard curve and averaged. Samples with mRNA levels below the lowest standard value, and thus below the detection limit, were given one-half the value of this lowest standard. Results are expressed as fold-change of control. Foldchanges $<1$ are reported as their negative inverse.

\section{Gelatin zymography on colonic mucosal samples}

Gelatin zymography was performed to quantify gelatinase activity of MMP2 and 9, as described previously ${ }^{(24)}$. MMP10 and 13 were not visible on this zymogram, because these MMP are collagenases. Moreover, MMP9 in particular is considered important in animal colitis research ${ }^{(25-27)}$. Colonic mucosal scrapings were loaded on the gels (1:1 diluted with sample buffer). Proteolytic activities were visualized by clear zones indicating lysis of gelatin. Quantification was performed using a Sharp Jx-330 scanner 
and Imagemaster ID software (Amersham Pharmacia) and enzyme activity was expressed as arbitrary unit/mg protein on the basis of lysed area.

\section{Statistical analysis}

The study was powered to detect a difference of $65 \%$ in MPO levels with an assumed SD of $40 \%$. For a power of $90 \%$ and an $\alpha$ of $5 \%$, the sample size needed was 8 rats per group. All results are expressed as mean \pm SEM. Our predefined main comparison of interest was HLA-B27 rats fed the control diet compared with HLA-B27 rats fed the calcium diet. Nontransgenic rats were included in this study as a noncolitic reference to provide some general comparative information on baseline values of the measured variables. Accordingly, statistics were conducted on nontransgenic rats compared with transgenic rats, each considered as 1 group, to test the effect of inflammation. Control transgenic rats were compared with calcium-fed transgenic rats to test the effect of dietary calcium. Normally distributed data were analyzed using Student's t test, whereas nonnormally distributed data were analyzed by Mann-Whitney U test (all 2 sided). Normality was tested by Kolmogorov-Smirnov test. All data were normally distributed except for the histological score and quantitative real-time PCR (Q-PCR) results of MMP9, MMP10, and MMP13. Differences were considered significant when $\mathrm{P}<0.05$. Statistical analyses were conducted with Graphpad software.

\section{RESULTS}

\section{Rats, food intake, and macroscopic evaluation of the colon}

Food intake of the transgenic rats remained stable during the experiment $(10.7 \pm 0.2 \mathrm{~g} / \mathrm{d})$ and did not differ between diet groups. Diet did not affect body weight gain $(1.0 \pm 0.1$ $\mathrm{g} / \mathrm{d})$ of the transgenic rats. Transgenic rats gained less weight $(1.7 \pm 0.1 \mathrm{~g} / \mathrm{d} ; \mathrm{P}<0.001)$ and had a lower food consumption $(11.8 \pm 0.4 \mathrm{~g} / \mathrm{d}$; $\mathrm{P}<0.05)$ than nontransgenic rats. Colons of transgenic rats fed the control diet $(13.7 \pm 0.1 \mathrm{~cm})$ were shorter than those fed the calcium supplemented diet $(14.4 \pm 0.3 \mathrm{~cm} ; \mathrm{P}<0.05)$. The dietary intervention did not influence colon weight in the transgenic rats $(2.1 \pm 0.03 \mathrm{~g})$, although it was much higher than that of nontransgenic rats $(1.0 \pm 0.1 \mathrm{~g}$; $\mathrm{P}<0.0001)$, a typical feature of chronic intestinal inflammation.

\section{Effect of dietary calcium on diarrhea and intestinal permeability}

Diarrhea is a prominent feature of colitis and can be quantified by determination of relative fecal wet weight. Dietary calcium protected transgenic rats against the increase in relative fecal wet weight from wk 4 onwards (Figure $1 ; \mathrm{P}<0.01$ ). Another major characteristic of IBD is an increased intestinal permeability. Within the first week of the experiment, intestinal permeability of the calcium-fed HLA-B27 rats was lower compared with transgenic rats fed the control diet (Figure 2; $\mathrm{P}<0.05$ ). Beginning at wk 5 , intestinal permeability of the control transgenic rats became even more different from calcium-fed HLA-B27 rats $(\mathrm{P}<0.01)$, indicating aggravation of intestinal inflammation (Figure 2). To determine whether an increased intestinal permeability indeed affects 


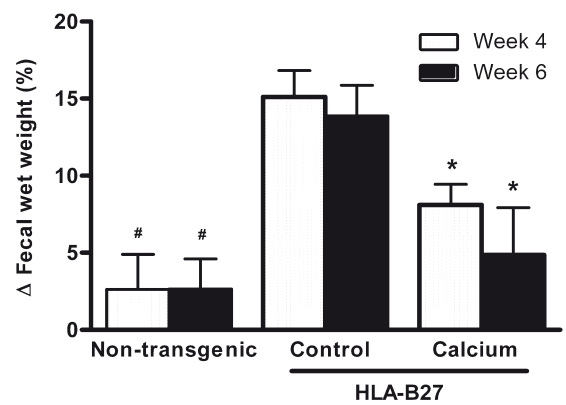

Figure 1: Effect of dietary calcium on the change in relative fecal wet weight in wk 4 and 6 compared with wk 2 in HLA-B27 transgenic rats. Results are means $\pm S E M, n=7-9$. *Different from control transgenic rats, $P<0.01$; ${ }^{\#}$ different from transgenic rats, $P<0.05$.

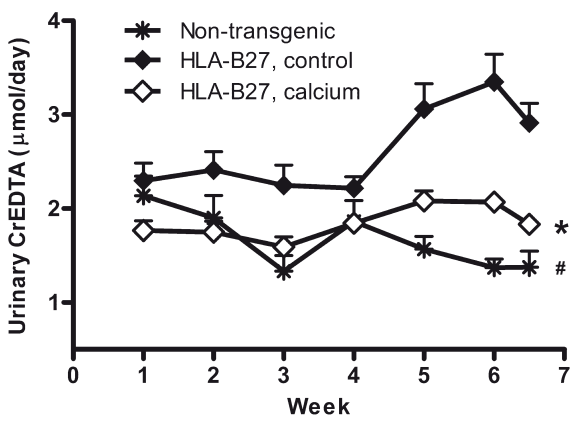

Figure 2: Effect of dietary calcium on urinary CrEDTA excretion, a marker for intestinal permeability, in HLA-B27 transgenic rats. Results are means \pm SEM, $n=7-9$. *Different from control transgenic rats at all time points, $P<0.05$; ${ }^{*}$ different from transgenic rats at wk 3, 5, 6, and 6.5, $P<0.05$.

the amount of luminal antigens entering the systemic circulation, we measured antibodies against LPS in serum. The increase in antibody titer from wk 0 to the end of the experiment tended to be more pronounced $(\mathrm{P}=0.09)$ in the transgenic rats of the control group (336 $\pm 111 \mathrm{kAU} / \mathrm{L})$ than the calcium-supplemented HLA-B27 rats (119 \pm $39 \mathrm{kAU} / \mathrm{L}$ ). This is consistent with the results obtained with the intestinal permeability marker CrEDTA. These variables were correlated $\left(\mathrm{R}^{2}=0.29 ; \mathrm{P}=0.03\right)$, but rat numbers are small, so we interpret these results with caution.

\section{Effect of dietary calcium on mucosal inflammation}

As dietary calcium exerted beneficial effects on diarrhea and intestinal permeability, we were interested in whether the accompanying intestinal inflammation was also affected by supplemental calcium. Intestinal inflammation was determined histologically and by measuring colonic mucosal MPO and IL-1 $\beta$ protein levels. Histological inflammation scores tended to be lower in the calcium-fed transgenic rats than in the transgenic rats fed the control diet (Figure 3; P=0.08). Although colonic mucosal MPO levels were much higher in the HLA-B27 rats compared with the nontransgenic rats $(1.4 \pm 0.2 \mathrm{ng} / \mathrm{mg}$ protein; $\mathrm{P}<0.0001$ ), diet did not influence the MPO concentration in the transgenic rats (control diet, $337.2 \pm 21.8 \mathrm{ng} / \mathrm{mg}$ protein; calcium diet, $298.3 \pm 27.1 \mathrm{ng} / \mathrm{mg}$ protein). However, IL-1 $\beta$ levels were lower in the calcium-supplemented transgenic rats (Figure 3; $\mathrm{P}<0.05$ ) compared with the control transgenic rats.

\section{Dietary calcium influences luminal cytotoxicity}

To verify whether calcium has luminal cytoprotective effects, we measured the cytotoxicity of fecal water. The cytotoxic activity of fecal water prepared from feces collected in wk 2 was significantly lower in transgenic rats fed the calcium diet $(5.6 \pm$ $1.9 \%)$ than in the transgenic rats fed the control diet $(21.5 \pm 5.8 \% ; \mathrm{P}<0.05)$. However, 

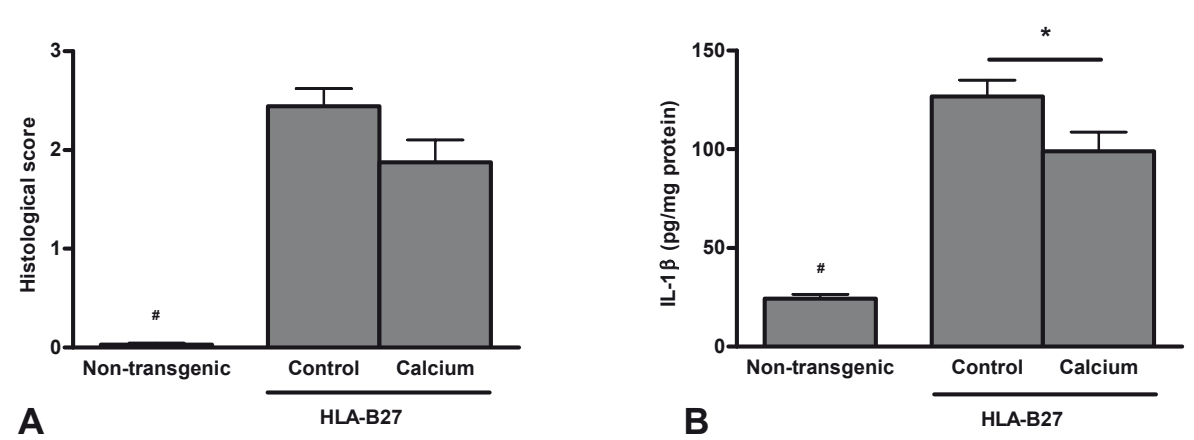

Figure 3: Effect of dietary calcium on inflammation in HLA-B27 transgenic rats. (A) Effect of calcium on histological score (0-4). (B) Effect of calcium on colonic mucosal IL-1ß levels. Results are means \pm SEM, $n=7-9$ ${ }^{*}$ Different from control transgenic rats, $P<0.05$; ${ }^{*}$ different from transgenic rats, $P<0.0001$.

this effect was not significant in wk 6 (data not shown). As calcium might also influence the intestinal epithelium directly, we checked the differences in calcium concentration of fecal water due to calcium supplementation. Indeed, the total calcium concentration in fecal water in wk 2 was higher in the calcium-fed HLA-B27 rats $(10.9 \pm 0.8 \mathrm{mmol} / \mathrm{L})$ than in the control transgenic rats $(5.5 \pm 0.6 \mathrm{mmol} / \mathrm{L} ; \mathrm{P}<0.0001)$. The difference in calcium concentration was similar in wk 6 (data not shown). However, luminal cytotoxicity and the calcium concentration in fecal water were not correlated $\left(\mathrm{R}^{2}=0.17 ; \mathrm{P}=0.10\right)$.

\section{Identification of colonic mucosal genes affected by dietary calcium}

After demonstrating that dietary calcium affected some major features of colitis development, we wanted to obtain more insight into the mechanisms playing a role. To achieve this in an unbiased way, whole-genome microarray analyses were performed on colonic mucosal samples of individual transgenic rats. The signal intensity of 28,213 spots was $>2$ times the background value and these spots were included in the analysis. Calcium supplementation changed the expression of 709 transcripts in colonic mucosa of transgenic rats $(\mathrm{P}<0.01)$. The set of 709 transcripts was subsequently used for pathway analysis. Dietary calcium clearly regulated one biological process: extracellular matrix remodeling $\left(\mathrm{P}<10^{-22} ; 22\right.$ genes of the 60 genes classified in this map in MetaCore were regulated, whereas the second-ranked process had a $\mathrm{P}<10^{-8}$ ).

Because not all genes are annotated in pathway programs, we manually classified the genes into processes using biological databases. For this purpose, we used unique genes that were regulated at least 1.3-fold of control with a P-value $<0.05$ (212 genes) and we specified processes only when at least 3 regulated genes were classified (Figure 4). Differentially regulated genes related to the process of extracellular matrix remodeling are shown in Table 2 . All genes affected in this process consistently had lower expression levels in the calcium-fed transgenic rats compared with the HLA-B27 rats fed the control diet, whereas expression of these genes is generally increased in IBD. The remainder of the 212 genes is shown in Supplemental Table 2. This additional analysis emphasized 


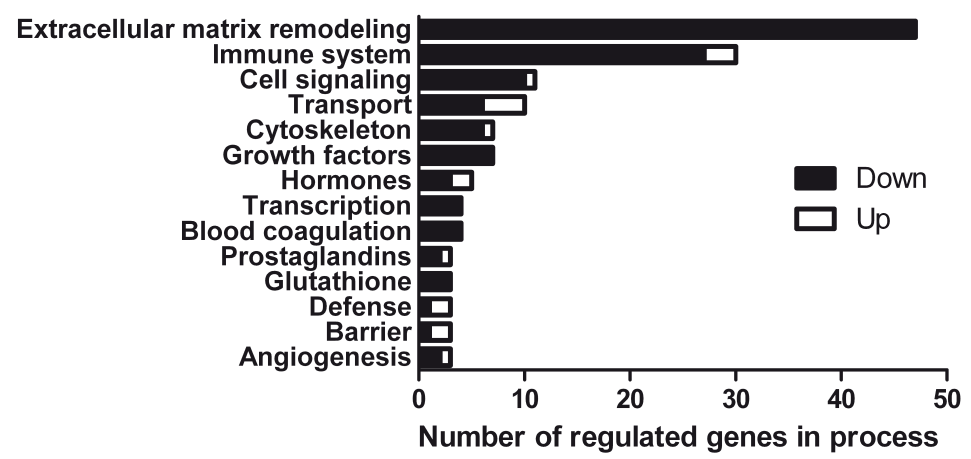

Figure 4: The number of regulated genes in colonic mucosa of transgenic HLA-B27 rats fed the calcium diet $(n=8)$ compared with control transgenic HLA-B27 rats $(n=9)$ classified in different processes. Genes included showed an absolute fold-change of at least 1.3 of control and a P-value $<0.05$. Only processes with at least 3 regulated genes are mentioned.

that extracellular matrix remodeling is the most prominently regulated process by calcium supplementation, followed by genes involved in the immune system. Moreover, calcium mostly downregulated (or induced less upregulation of) these genes in the transgenic rats.

Noteworthy is the fact that calcium had a small but significant effect on some important mucosal barrier genes. Dietary calcium upregulated the mucin MUC2 (foldchange=1.2; $\mathrm{P}=0.002$ ), trefoil factor 1 (fold-change=1.3; $\mathrm{P}=0.009$ ), and trefoil factor 3 (fold-change=1.2; $\mathrm{P}=0.011$ ). Interestingly, the calcium diet slightly downregulated cyclooxygenase 2 (ptgs2/cox2; fold-change=-1.3; $\mathrm{P}=0.012$ ).

\section{Confirmation of regulated genes by Q-PCR in transgenic and nontransgenic rats}

To confirm microarray data, mRNA expression of a subset of selected genes was analyzed by Q-PCR. MMP9, MMP10, MMP13, procollagen type V $\alpha 2$ (Col5a2) and fibronectin 1 (Fn1) were measured as important representatives for the extracellular matrix remodeling process. Q-PCR analysis for all genes confirmed the microarray data (Supplemental Figure 1); dietary calcium decreased mRNA expression of MMP9 (foldchange=-3.4), MMP10 (fold-change=-5.4), MMP13 (fold-change=-6.1), Col5a2 (foldchange $=-1.6$ ), and Fn1 (fold-change $=-3.0$ ). As expected, expression of all MMP analyzed, Col5a2, and Fn1 was low in colonic mucosa of the nontransgenic rats.

\section{Effect of dietary calcium on mucosal MMP activity}

To show that the influence of dietary calcium on extracellular matrix remodeling was also apparent on the protein level, we performed quantitative gelatin zymography. Clearly, the colonic mucosal scrapings contained active gelatinases of varying molecular weight, namely proMMP9, proMMP2, and MMP2 (Figure 5A). The lytic band of $\sim 80 \mathrm{kDa}$ represents some unidentified proteolytic activity, which is not MMP9 ${ }^{(24)}$. Importantly, 
Table 2: Differentially regulated genes related to extracellular matrix remodeling in response to the calcium diet in HLA-B27 transgenic rats

\begin{tabular}{|c|c|c|c|}
\hline Gene name & Gene symbol & Accession по. & Fold-change \\
\hline MMP13 & Mmp13 & XM_001072242 & -4.3 \\
\hline MMP10 & Mmp10 & NM_133514 & -3.7 \\
\hline MMP9 & Mmp9 & NM_031055 & -2.5 \\
\hline MMP3 & Mmp3 & NM_133523 & -2.1 \\
\hline Decorin & Dcn & NM_024129 & -2.0 \\
\hline Matrix Gla protein & Mgp & NM_012862 & -1.8 \\
\hline Fn1 & Fn1 & NM_019143 & -1.8 \\
\hline Nidogen 1 & Nid1 & XM_213954 & -1.8 \\
\hline Protease, serine, 22 & Prss22_predicted & XM_220222 & -1.8 \\
\hline Thrombospondin 2 & Thbs2 & XM_214778 & -1.7 \\
\hline Transglutaminase 1 & $\operatorname{Tgm} 1$ & NM_031659 & -1.7 \\
\hline Cell adhesion molecule with homology to L1CAM & Chl1 & XM_001077843 & -1.7 \\
\hline Lumican & Lum & NM_031050 & -1.7 \\
\hline Glycoprotein (transmembrane) $\mathrm{nmb}$ & Gpnmb & NM_133298 & -1.6 \\
\hline Serine protease inhibitor B3 & Serpinb3 & NM_001008887 & -1.5 \\
\hline Col5a2 & Col5a2 & XM_343564 & -1.5 \\
\hline Connective tissue growth factor & Ctgf & NM_022266 & -1.5 \\
\hline $\begin{array}{l}\text { Serine (or cysteine) proteinase inhibitor, clade } \\
\quad \text { E, member } 2\end{array}$ & Serpine2 & XM_343604 & -1.5 \\
\hline Similar to Laminin $\alpha-4$ chain precursor & $\begin{array}{l}\text { RGD1560062 } \\
\text { predicted }\end{array}$ & XM_228209 & -1.5 \\
\hline Plasminogen activator, urokinase & Plau & NM_013085 & -1.5 \\
\hline $\begin{array}{l}\text { Melanoma cell adhesion molecule, transcript } \\
\text { variant } 1\end{array}$ & Mcam & NM_023983 & -1.5 \\
\hline Mesothelin & Msln & NM_031658 & -1.4 \\
\hline HtrA serine peptidase 1 & Htra1 & NM_031721 & -1.4 \\
\hline Procollagen, type l, $\alpha 1$ & Col1a1 & XM_213440 & -1.4 \\
\hline Procollagen, type IV, $\alpha 1$ & Col4a1 & XM_214400 & -1.4 \\
\hline Laminin, $\beta 1$ & Lamb1_predicted & XM_216679 & -1.4 \\
\hline Biglycan & Bgn & NM_017087 & -1.4 \\
\hline Periostin, osteoblast specific factor & Postn_predicted & XM_342245 & -1.4 \\
\hline Sulfatase 1 & Sulf1 & NM_134378 & -1.4 \\
\hline Procollagen, type I, $\alpha 2$ & Col1a2 & NM_053356 & -1.4 \\
\hline Podocalyxin-like & Podxl & NM_138848 & -1.4 \\
\hline
\end{tabular}


Table 2 continued

\begin{tabular}{lclc}
\hline Gene name & Gene symbol & Accession no. & Fold-change \\
\hline MMP2 & Mmp2 & U65656 & -1.4 \\
Procollagen, type XVI, $\alpha 1$ & Col16a1 & XM_345584 & -1.4 \\
MMP8 & Mmp8 & NM_022221 & -1.3 \\
Procollagen C-endopeptidase enhancer protein & Pcolce & NM_019237 & -1.3 \\
$\begin{array}{l}\text { Serine (or cysteine) peptidase inhibitor, clade E, } \\
\text { member 1 }\end{array}$ & Serpine1 & NM_012620 & -1.3 \\
Secreted acidic cysteine rich glycoprotein & Sparc & NM_012656 & -1.3 \\
Lysyl oxidase & Lox & NM_017061 & -1.3 \\
MMP7 & Mmp7 & NM_012864 & -1.3 \\
Similar to a disintegrin-like and metalloprotease & Adamts9_predicted & XM_232202 & -1.3 \\
$\quad$ with thrombospondin type 1 motifs 9B & & & \\
Chondroitin sulfate proteoglycan 2 & Cspg2 & XM_215451 & -1.3 \\
Cathepsin L & Ctsl & NM_013156 & -1.3 \\
Laminin, $\alpha 5$ & Lama5 & XM_215963 & -1.3 \\
Procollagen, type III, $\alpha 1$ & Col3a1 & NM_032085 & -1.3 \\
Procollagen, type XV & Col15a1 & XM_216399 & -1.3 \\
Lysyl oxidase-like 2 & Loxl2_predicted & XM_214225 & -1.3 \\
Serine (or cysteine) proteinase inhibitor, clade & Serpinh1 & NM_017173 & -1.3 \\
$\quad$ H, member 1 & & & \\
\hline & & & \\
\hline
\end{tabular}

Results are genes related to extracellular matrix remodeling that are differentially regulated at least 1.3-fold with a $P$-value $<0.05$ in colonic mucosa of transgenic rats fed the calcium diet $(n=8)$ compared with the transgenic rats fed the control diet $(n=9)$. Genes are sorted based on absolute fold-change of control. Fold changes $<1$ are reported as their negative inverse. Note that all genes related to extracellular matrix remodeling are downregulated by the calcium diet relative to the control diet in the HLA-B27 rats.

proMMP2 and MMP2 activity were lower in transgenic rats fed the calcium diet compared to the control transgenic rats (Figure 5B; $\mathrm{P}<0.05$ ), confirming the MMP mRNA results on the protein level. ProMMP9 activity did not differ in transgenic rats fed the calcium $\operatorname{diet}(7.5 \pm 0.7$ arbitrary unit/mg protein) compared with control transgenic rats (8.4 \pm 0.7 arbitrary unit/mg protein), showing that mRNA data are not always reflected on the protein level.

\section{DiscuSSION}

The present study shows that dietary calcium inhibits important aspects of colitis severity in HLA-B27 transgenic rats. Dietary calcium effectively prevented the increase in intestinal permeability and diminished diarrhea, both well-known characteristics of 
IBD. Moreover, mucosal IL-1 $\beta$ levels were lower in calciumsupplemented rats and the histological inflammation score tended to be lower, which coincided with decreased extracellular matrix breakdown in colonic mucosa.

The beneficial effect of calcium on intestinal permeability is particularly very interesting, because an increased permeability is suggested to be one of the first events to occur in the development of IBD in humans and precedes relapse of disease ${ }^{(28-30)}$. In this study, intestinal permeability increased from wk 5 in transgenic rats fed the control diet. In contrast, intestinal permeability of the transgenic rats fed the calcium-supplemented diet remained stable. As the intestines are always permeable to some extent, the presence or absence of colitis results from the fact that the transgenic rats react much more strongly to (the same amount of) translocating luminal antigens compared with the nontransgenic rats because of their overreactive immune system.

Although we cannot definitely conclude from our study that an increase in intestinal permeability preceded colonic inflammation, it is tempting to speculate that dietary calcium conferred at least partial protection against inflammation by preserving gut barrier function. It is well known that the gut microbiota and its close intestinal contact with the overreactive immune system is a driving force for chronic inflammation in $\mathrm{IBD}^{(1)}$. Improvement of gut barrier function by dietary calcium, thereby decreasing translocation of intestinal bacteria or bacterial antigens, might be a highly attractive and safe supplemental intervention to be further explored in IBD patients.

Influencing intestinal permeability might be part of the protective effect, but the molecular mechanism behind the beneficial effect of calcium on colitis severity is far from clear. Previous studies demonstrated that calcium forms an insoluble complex with phosphate in the upper small intestine ${ }^{(31,32)}$. This amorphous calcium phosphate binds luminal bile acids and fatty acids, thereby limiting solubility of these irritating substances and resulting in less damage to delicate colonic epithelial cells. Indeed, in the present study, calcium decreased cytotoxicity of intestinal contents in accordance
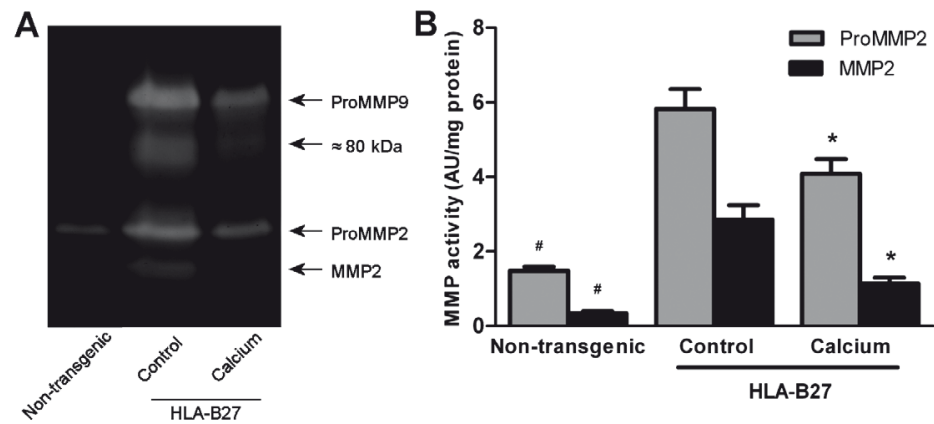

Figure 5: Effect of dietary calcium on MMP activity in colonic mucosa. (A) Example of MMP activity as analyzed by gelatin zymography. Representative samples are shown for nontransgenic rats (n=7) and HLA-B27 transgenic rats fed the control diet $(n=9)$ and calcium diet $(n=8)$. (B) Effect of calcium on proMMP2 and MMP2 activity indicated in arbitrary unit $(A U) / m g$ protein. Results are means \pm SEM. ${ }^{*}$ Different from control transgenic rats, $P<0.05$; "different from transgenic rats, $P<0.005$. 
with earlier results ${ }^{(6,7)}$.

To gain more insight into the effect of dietary calcium on mucosal integrity, total colonic mucosal gene expression was analyzed. It should be realized that gene expression changes are generally small in nutritional interventions ${ }^{(33)}$. However, by combining the small effects of significantly changed genes into common biological pathways, physiologically relevant modulated processes can be identified ${ }^{(34)}$. In this study, many genes involved in extracellular matrix remodeling were consistently less upregulated in the calcium-fed transgenic rats, especially several MMP and extracellular matrix components such as procollagens and fibronectin. Under physiological conditions, MMP are counterbalanced by tissue inhibitors of MMP; however, no dietary effect was observed on gene expression of these inhibitors. Gelatin zymography of colonic mucosal samples was performed to quantify the actual activity of a particular category of MMP and showed that MMP2 activity was indeed lower in transgenic rats fed the calcium diet compared with the transgenic control rats. Evidence is growing that MMP are implicated in intestinal inflammatory disorders and contribute to tissue damage ${ }^{(35)}$. Biopsies of IBD patients show elevated levels of $\mathrm{MMP}^{(36,37)}$. Also, the collagen content is increased in the intestinal submucosa of Crohn's disease patients compared with controls $^{(38)}$. However, we speculate that the effect of calcium on extracellular matrix remodeling is not necessarily the result of the diminished inflammatory response but rather a consequence of the beneficial effect of dietary calcium on intestinal permeability. Lowering intestinal permeability reduces exposure of the colonic mucosa to luminal antigens, likely resulting in decreased inflammation and coinciding extracellular matrix remodeling. Whether intestinal permeability remained low because of the reduced luminal cytotoxicity due to calcium is not known and will be further investigated. Direct effects of calcium on mucosal processes due to different colonic mucosal calcium concentrations are not expected, because calcium absorption is very tightly regulated and mainly occurs in the proximal small intestine ${ }^{(39)}$. Also, systemic (serum) calcium concentrations are extremely stable and are not influenced by dietary calcium supplementation ${ }^{(40)}$. On the other hand, the observed difference in the luminal calcium concentration due to our dietary intervention might affect gut barrier function more directly. Obviously, alterations in tight junction proteins as the primary regulators of intestinal permeability are expected. Although calcium did not induce changes in gene expression of tight junction proteins (e.g. claudins, occludin, zona occludens, and myosin IXb), morphological and structural alterations in tight junctions might exist. For example, internalization into the cellular cytoplasm and phosphorylation of tight junction proteins are suggested to be important for leakiness of the mucosal barrier ${ }^{(41)}$. Extracellular (luminal) calcium is crucial for maintenance of intestinal tight junction function in cell studies. However, these effects seem to occur at free calcium concentrations up to $0.1 \mathrm{mmol} / \mathrm{L}^{(42)}$, which is much lower than the luminal calcium levels observed in our study. Another possibility, which will be further explored, is that calcium influences the viscosity of the mucus layer, thereby altering intestinal permeability ${ }^{(43)}$.

Interestingly, IL-1 $\beta$ levels were lower in calcium-fed rats and the histological colitis score tended to be lower compared with the control transgenic rats, whereas mucosal MPO 
levels did not differ between the groups. This might be explained by the fact that the inflammatory cell infiltrate in the mucosa of the HLAB27 transgenic rats predominantly consists of mononuclear cells and eosinophils, in which MPO is not as abundantly present as in neutrophils ${ }^{(17)}$. Although MPO is often considered the golden standard to quantify inflammation, in this rat model, measurement of mucosal MPO might be less accurate and underestimate the antiinflammatory effect of calcium.

As far as we know, 2 studies reported on the possible protective effects of dietary calcium on intestinal inflammation. Zhu et al. ${ }^{(8)}$ found ameliorating effects on gut inflammation in IL-10 knockout mice, but this study mainly focused on the role of vitamin D in IBD. The recent work of Pele et al. ${ }^{(9)}$ showed that dietary calcium protected against dextran sulfate sodium (DSS)-induced colitis in mice, but these results should be interpreted with caution, because DSS is precipitated by calcium (information of the manufacturer). They tried to address this issue by applying a rather indirect analysis focusing on macrophages, whereas luminal exposure of epithelial cells to DSS was not included in this assay. Intestinal precipitation of orally administered DSS by calcium induces unwanted differences in exposure of the mice to the colitis-inducing agent. A major advantage of the HLA-B27 transgenic rat model is its independence of chemical induction, thereby excluding a direct interactionbetween the nutritional or pharmacological intervention and the chemical needed to induce colitis.

To mimic the composition of a human Western-type diet and facilitate extrapolation to the human situation, our rat diets had a relatively high-fat content compared with standard rodent diets ${ }^{(4)}$. Because such a small amount of the supplemented calcium intake is absorbed (generally $<10 \%)^{(6)}$, recalculation of the calcium dose and expression per kg body weight is of little relevance. Therefore, the calcium content in the control diet (30 mmol or $1.2 \mathrm{~g} / \mathrm{kg}$ dry food) corresponds to a daily calcium intake of $600 \mathrm{mg}$ in humans, assuming that humans have a daily dry food intake of $\sim 500 \mathrm{~g}$. In general, human dietary calcium intake in the Western world ranges from $600-1100 \mathrm{mg} / \mathrm{d}^{(45)}$. So, the control diet matches with the lower limit of the human intake range, whereas the calcium-supplemented diet (comparable to $2.4 \mathrm{~g} / \mathrm{d}$ ) provided more than the general habitual dietary calcium intake. However, this is not an unrealistic intake when using calcium supplements.

In conclusion, the present study shows that dietary calcium inhibits important aspects of colitis severity in HLA-B27 transgenic rats. Clinical trials are required and planned to determine whether supplemental calcium has similar beneficial effects in IBD patients. The results of the present study point to the usefulness of nutrition in modulating gut mucosal integrity. In contrast to medication, the lack of undesired side effects of a nutritional intervention is a major advantage. We realize that nutrition cannot replace medication; however, it might be useful as a supporting therapeutic strategy. Moreover, the protective effects of dietary calcium are not restricted to inflammation in IBD. As mentioned above, supplemental calcium protects against intestinal infections and colon carcinogenesis. This suggests that calcium in the gut affects some generic luminal mechanisms, thereby protecting against the development of several intestinal diseases. 


\section{Acknowledgments}

We thank Wilma Blauw (Small Animal Centre, Wageningen University and Research Centre, The Netherlands) and Roger Lomme (Department of Surgery, Radboud University Nijmegen Medical Centre, The Netherlands) for their expert assistance and Charles Slangen (NIZO Food Research, Ede, The Netherlands) for statistical advice.

\section{REFERENCES}

1. Sartor RB. Mechanisms of disease: pathogenesis of Crohn's disease and ulcerative colitis. Nat Clin Pract Gastroenterol Hepatol. 2006;3:390-407.

2. Ling SC, Griffiths AM. Nutrition in inflammatory bowel disease. Curr Opin Clin Nutr Metab Care. 2000;3:339-44.

3. Bovee-Oudenhoven IM, Lettink-Wissink ML, Van Doesburg W, Witteman BJ, Van Der Meer R. Diarrhea caused by enterotoxigenic Escherichia coli infection of humans is inhibited by dietary calcium. Gastroenterology. 2003;125:469-76.

4. Bovee-Oudenhoven IM, Termont DS, Weerkamp AH, Faassen-Peters MA, Van der Meer R. Dietary calcium inhibits the intestinal colonization and translocation of Salmonella in rats. Gastroenterology. 1997; 113:550-7.

5. Ten Bruggencate SJ, Bovee-Oudenhoven IM, Lettink-Wissink ML, Katan MB, Van Der Meer R. Dietary fructo-oligosaccharides and inulin decrease resistance of rats to salmonella: protective role of calcium. Gut. 2004;53:530-5.

6. Govers MJ, Termont DS, Lapre JA, Kleibeuker JH, Vonk RJ, Van der Meer R. Calcium in milk products precipitates intestinal fatty acids and secondary bile acids and thus inhibits colonic cytotoxicity in humans. Cancer Res. 1996;56:3270-5.

7. Lapre JA, De Vries HT, Koeman JH, Van der Meer R. The antiproliferative effect of dietary calcium on colonic epithelium is mediated by luminal surfactants and dependent on the type of dietary fat. Cancer Res. 1993;53:784-9.

8. Zhu Y, Mahon BD, Froicu M, Cantorna MT. Calcium and 1 alpha,25-dihydroxyvitamin D3 target the TNFalpha pathway to suppress experimental inflammatory bowel disease. Eur J Immunol. 2005; 35:217-24.

9. Pele LC, Thoree V, Mustafa F, He S, Tsaprouni L, Punchard NA, Thompson RP, Evans SM, Powell JJ. Low dietary calcium levels modulate mucosal caspase expression and increase disease activity in mice with dextran sulfate sodium induced colitis. J Nutr. 2007;137: 2475-80.

10. Sartor RB. Colitis in HLA-B27/beta 2 microglobulin transgenic rats. Int Rev Immunol. 2000;19:39-50.

11. Qian BF, Tonkonogy SL, Sartor RB. Aberrant innate immune responses in TLR-ligand activated HLA-B27 transgenic rat cells. Inflamm Bowel Dis. 2008;14:1358-65.

12. Arslan G, Atasever T, Cindoruk M, Yildirim IS. (51)CrEDTA colonic permeability and therapy response in patients with ulcerative colitis. Nucl Med Commun. 2001;22:997-1001.

13. Binnerts W, Van het Klooster A, Frens A. Soluble chromium indicator measured by atomic absorption in digestion experiments. Vet Rec. 1968;82:470.

14. Sesink AL, Termont DS, Kleibeuker JH, Van der Meer R. Red meat and colon cancer: the cytotoxic and hyperproliferative effects of dietary heme. Cancer Res. 1999;59:5704-9.

15. Bovee-Oudenhoven I, Termont D, Dekker R, Van der Meer R. Calcium in milk and fermentation by yoghurt bacteria increase the resistance of rats to Salmonella infection. Gut. 1996;38:59-65.

16. Lapre JA, Termont DS, Groen AK, Van der Meer R. Lytic effects of mixed micelles of fatty acids and bile acids. Am J Physiol. 1992;263: G333-7.

17. Rath HC, Herfarth HH, Ikeda JS, Grenther WB, Hamm TE Jr, Balish E, Taurog JD, Hammer RE, Wilson $\mathrm{KH}$, et al. Normal luminal bacteria, especially Bacteroides species, mediate chronic colitis, gastritis, and arthritis in HLA-B27/human beta2 microglobulin transgenic rats. J Clin Invest. 1996;98:945-53.

18. Dieleman LA, GoerresMS, Arends A, Sprengers D, Torrice C, Hoentjen F, Grenther WB, Sartor RB. Lactobacillus GG prevents recurrence of colitis in HLA-B27 transgenic rats after antibiotic treatment. Gut. 2003;52: 370-6.

19. Wettenhall JM, Smyth GK. limmaGUI: a graphical user interface for linear modeling of microarray data. 
Bioinformatics. 2004;20:3705-6.

20. Pellis L, Franssen-van Hal NL, Burema J, Keijer J. The intraclass correlation coefficient applied for evaluation of data correction, labeling methods, and rectal biopsy sampling in DNA microarray experiments. Physiol Genomics. 2003;16:99-106.

21. Lee HK, Braynen W, Keshav K, Pavlidis P. ErmineJ: tool for functional analysis of gene expression data sets. BMC Bioinformatics. 2005;6:269.

22. Rodenburg W, Keijer J, Kramer E, Roosing S, Vink C, Katan MB, van der Meer R, Bovee-Oudenhoven IM. Salmonella induces prominent gene expression in the rat colon. BMC Microbiol. 2007;7:84.

23. Vandesompele J, De Preter K, Pattyn F, Poppe B, Van Roy N, De Paepe A, Speleman F. Accurate normalization of real-time quantitative RT-PCR data by geometric averaging of multiple internal control genes. Genome Biol. 2002;3:RESEARCH0034.

24. de Hingh $\mathrm{IH}$, Lomme RM, van Goor H, Bleichrodt RP, Hendriks T. Changes in gelatinase activity in the gastrointestinal tract after anastomotic construction in the ileum or colon. Dis Colon Rectum. 2005;48:2133-41.

25. Medina C, Santana A, Paz MC,DiazF, Farre E,Salas A, Radomski MW, Quintero E. Matrix metalloproteinase-9 modulates intestinal injury in rats with transmural colitis. J Leukoc Biol.2006;79:954-62.

26. Castaneda FE, Walia B, Vijay-Kumar M, Patel NR, Roser S, Kolachala VL, Rojas M, Wang L, Oprea G, et al. Targeted deletion of metalloproteinase 9 attenuates experimental colitis in mice: central role of epithelial-derived MMP. Gastroenterology. 2005;129:1991-2008.

27. Santana A,Medina C, Paz-CabreraMC, Diaz-Gonzalez F, Farre E, Salas A, Radomski MW, Quintero E. Attenuation of dextran sodium sulphate induced colitis in matrix metalloproteinase- 9 deficient mice. World J Gastroenterol. 2006;12:6464-72.

28. Arrieta MC, Bistritz L, Meddings JB. Alterations in intestinal permeability. Gut. 2006;55:1512-20.

29. Arnott ID, Kingstone K, Ghosh S. Abnormal intestinal permeability predicts relapse in inactive Crohn disease. Scand J Gastroenterol. 2000;35:1163-9.

30. Wyatt J, Vogelsang H, Hubl W, Waldhoer T, Lochs H. Intestinal permeability and the prediction of relapse in Crohn's disease. Lancet. 1993;341:1437-9.

31. Govers MJ, Van der Meer R. Effects of dietary calcium and phosphate on the intestinal interactions between calcium, phosphate, fatty acids, and bile acids. Gut. 1993;34:365-70.

32. Van der Meer R, Welberg JW, Kuipers F, Kleibeuker JH, Mulder NH, Termont DS, Vonk RJ, De Vries HT, De Vries EG. Effects of supplemental dietary calcium on the intestinal association of calcium, phosphate, and bile acids. Gastroenterology. 1990;99:1653-9.

33. Afman L, Muller M. Nutrigenomics: from molecular nutrition to prevention of disease. J Am Diet Assoc. 2006;106:569-76.

34. Rodenburg W, Heidema AG, Boer JM, Bovee-Oudenhoven IM, Feskens EJ, Mariman EC, Keijer J. A framework to identify physiological responses in microarray-based gene expression studies: selection and interpretation of biologically relevant genes. Physiol Genomics. 2008;33:78-90.

35. Medina C, Radomski MW. Role of matrix metalloproteinases in intestinal inflammation. J Pharmacol Exp Ther. 2006;318:933-8.

36. Baugh MD, Perry MJ, Hollander AP, Davies DR, Cross SS, Lobo AJ, Taylor CJ, Evans GS. Matrix metalloproteinase levels are elevated in inflammatory bowel disease. Gastroenterology. 1999;117:81422.

37. von Lampe B, Barthel B, Coupland SE, Riecken EO, Rosewicz S. Differential expression of matrix metalloproteinases and their tissue inhibitors in colon mucosa of patients with inflammatory bowel disease. Gut. 2000;47:63-73.

38. Graham MF, Diegelmann RF, Elson CO, Lindblad WJ, Gotschalk N, Gay S, Gay R. Collagen content and types in the intestinal strictures of Crohn's disease. Gastroenterology. 1988;94:257-65.

39. Kodicek E. The absorption of calcium from the intestine. Proc Nutr Soc. 1967;26:67-72.

40. Appleton GV, Owen RW, Wheeler EE, Challacombe DN, Williamson RC. Effect of dietary calcium on the colonic luminal environment. Gut. 1991;32:1374-7.

41. Bruewer M, Samarin S, Nusrat A. Inflammatory bowel disease and the apical junctional complex. Ann $\mathrm{N}$ Y Acad Sci. 2006;1072:242-52.

42. Gonzalez-Mariscal L, Contreras RG, Bolivar JJ, Ponce A, Chavez De Ramirez B, Cereijido M. Role of calcium in tight junction formation between epithelial cells. Am J Physiol. 1990;259:C978-86. 
43. Crowther RS, Marriott C, James SL. Cation induced changes in the rheological properties of purified mucus glycoprotein gels. Biorheology. 1984;21:253-63.

44. Reeves PG, Nielsen FH, Fahey GC Jr. AIN-93 purified diets for laboratory rodents: final report of the American Institute of Nutrition ad hoc writing committee on the reformulation of the AIN-76A rodent diet. J Nutr. 1993;123:1939-51.

45. Alaimo K, McDowell MA, Briefel RR, Bischof AM, Caughman CR, Loria CM, Johnson CL. Dietary intake of vitamins, minerals, and fiber of persons ages 2 months and over in the United States: Third National Health and Nutrition Examination Survey, Phase 1,1988-91. Adv Data. 1994;258:1-28.

\section{SUPPLEMENTAL DATA}

Supplemental table 1: primer information

\begin{tabular}{lllll}
\hline $\begin{array}{l}\text { Gene } \\
\text { symbol }\end{array}$ & $\begin{array}{l}\text { Accession } \\
\text { number }\end{array}$ & Sense & Antisense & $\begin{array}{l}\text { T annealing } \\
\left({ }^{\circ} \mathrm{C}\right)\end{array}$ \\
\hline MMP9 & NM_031055 & AGAAGTGGGGTTTCTGTCCAGAC & GGAGCTGTCGGCTGTGGTTC & 59.0 \\
MMP10 & NM_133514 & TGTGATCCTGCTTTGTCCTTTGATG & CGGGATTCCACTGGGTTCTACG & 59.0 \\
MMP13 & XM_001072242 & AAGGCTCAGTGCTGCGGTTC & CATGACTCTCACAATGCGATTACTCC & 59.0 \\
Col5a2 & XM_343564 & CTGTTCAAAGCGAAATGGAAATGTAGG & TTCTTGTGTTGGTCATTGATGGTAGTC & 60.0 \\
Fn1 & NM_019143 & GACTACCACCTCTATCCTCATGTTCC & AACCTCTTCTCGGACCTTGTGC & 60.0 \\
B-actin & NM_031144 & CTTTCTACAATGAGCTGCGTGTG & GTCAGGATCTTCATGAGGTAGTCTGTC & 60.0 \\
Arf1 & NM_022518 & CTCCGAGATGCCGTTCTCTTG & GGTAGCCTGAATGTACCAGTTCC & 60.0 \\
Aldolase & NM_012495 & ATGCCCCACCCATACCCAGCACT & AGCAGCAGTTGGCGGTAGAAGCG & 60.0 \\
\hline
\end{tabular}

Supplemental table 2: Differentially regulated genes in colonic mucosa of transgenic HLA-B27 rats fed the calcium versus control diet classified in different processes

\begin{tabular}{|c|c|c|c|}
\hline Gene name & $\begin{array}{l}\text { Gene } \\
\text { symbol }\end{array}$ & $\begin{array}{l}\text { Accession } \\
\text { number }\end{array}$ & $\begin{array}{l}\text { Fold } \\
\text { change }\end{array}$ \\
\hline \multicolumn{4}{|l|}{ Immune system } \\
\hline $\begin{array}{l}\text { Similar to Fc fragment of IgG binding protein; IgG Fc binding } \\
\text { protein (LOC292746) }\end{array}$ & $X M_{-} 214835$ & XM_214835 & 1.5 \\
\hline Proteoglycan 2, bone marrow & $\operatorname{Prg} 2$ & NM_031619 & 1.3 \\
\hline Chemokine (C-X3-C motif) ligand 1 & Cx3cl1 & NM_134455 & 1.3 \\
\hline Triggering receptor expressed on myeloid cells 2 & Trem2 & XM_217335 & -2.0 \\
\hline $\begin{array}{l}\text { IgM kappa chain variable region \{CDR1 to CDR3 region\} [rats, } \\
\text { LEW, HAR-1 hybridoma cells, Partial, } 408 \mathrm{nt} \text { ] }\end{array}$ & S81289 & S81289 & -1.6 \\
\hline Bone marrow stromal cell antigen 1 & Bst1 & NM_030848 & -1.5 \\
\hline NGF-binding Ig rearranged kappa-chain, V-region, partial cds & $L 17080$ & L17080 & -1.5 \\
\hline S100 calcium binding protein A8 (calgranulin A) & S100a8 & NM_053822 & -1.5 \\
\hline Glycoprotein 49b & Gp49b & NM_001013894 & -1.5 \\
\hline Fc receptor, IgG, low affinity IIb & $F c g r 2 b$ & NM_175756 & -1.5 \\
\hline
\end{tabular}




\begin{tabular}{|c|c|c|c|}
\hline Gene name & $\begin{array}{l}\text { Gene } \\
\text { symbol }\end{array}$ & $\begin{array}{l}\text { Accession } \\
\text { number }\end{array}$ & $\begin{array}{l}\text { Fold } \\
\text { change }\end{array}$ \\
\hline Rat class III Fc-gamma receptor, complete cds & Fcgr3 & M64368 & -1.4 \\
\hline Complement component 5 , receptor 1 & C5r1 & NM_053619 & -1.4 \\
\hline Fc receptor, IgG, low affinity III & Fcgr3 & NM_053843 & -1.4 \\
\hline Interleukin 1 receptor-like 1 & Il1rl1 & NM_013037 & -1.4 \\
\hline Complement component 1 , s subcomponent & C1s & NM_138900 & -1.4 \\
\hline Predicted: similar to immunoglobulin light chain (LOC500166) & $X M \_575518$ & XM_575518 & -1.4 \\
\hline Interferon induced transmembrane protein 3 & Ifitm3 & XM_341957 & -1.4 \\
\hline CD48 antigen & Cd48 & NM_139103 & -1.4 \\
\hline Chemokine (C-C motif) ligand 11 & Ccl11 & NM_019205 & -1.4 \\
\hline Collectin sub-family member 12 & Colec12 & NM_001025721 & -1.4 \\
\hline Predicted: Fc receptor, IgG, low affinity III & Fcgr3 & XM_001077008 & -1.3 \\
\hline Macrophage-inducible C-type lectin & Clecsf 9 & NM_001005897 & -1.3 \\
\hline Chemokine orphan receptor 1 & Cmkor1 & NM_053352 & -1.3 \\
\hline $\begin{array}{l}\text { UI-R-FS1-cqk-h-23-0-UI.s1 UI-R-FS1 cDNA clone UI-R-FS1- } \\
\text { cqk-h-23-0-UI 3' }\end{array}$ & S100a8 & CA507495 & -1.3 \\
\hline Chemokine (C-C motif) receptor 1 & Ccr1 & NM_020542 & -1.3 \\
\hline Immunoglobulin superfamily, member 10 & $\operatorname{Igsf10}$ & NM_198768 & -1.3 \\
\hline Fc receptor, IgG, high affinity I & Fcgr1 & XM_215643 & -1.3 \\
\hline $\begin{array}{l}\text { Similar to gamma-2a immunoglobulin heavy chain } \\
\text { (LOC366758) }\end{array}$ & $X M \_345745$ & XM_345745 & -1.3 \\
\hline $\begin{array}{l}\text { Phospholipase A2, group VII (platelet-activating factor } \\
\text { acetylhydrolase, plasma) }\end{array}$ & Pla2g7 & NM_001009353 & -1.3 \\
\hline Complement component $1, \mathrm{r}$ subcomponent & C1r & XM_242644 & -1.3 \\
\hline \multicolumn{4}{|l|}{ Cell signaling } \\
\hline RAB3B, member RAS oncogene family & $R a b 3 b$ & NM_031091 & 1.4 \\
\hline Wnt inhibitory factor 1 & Wif1 & NM_053738 & -1.7 \\
\hline Tumor necrosis factor receptor superfamily, member $12 \mathrm{a}$ & Tnfrsf12a & NM_181086 & -1.3 \\
\hline $\begin{array}{l}\text { DDR2_MOUSE (Q62371) Discoidin domain receptor } 2 \\
\text { precursor (Receptor protein-tyrosine kinase TKT) } \\
\text { (Tyrosine-protein kinase TYRO 10) (Neurotrophic tyrosine } \\
\text { kinase, receptor-related 3) , partial (17\%) }\end{array}$ & TC519882 & TC519883 & -1.3 \\
\hline Neuregulin 1 & $\operatorname{Nrg} 1$ & NM_031588 & -1.3 \\
\hline $\begin{array}{l}\text { Predicted: protein tyrosine phosphatase, non-receptor type } \\
\text { substrate } 1 \text {-like } 3\end{array}$ & $\begin{array}{l}\text { Ptpns113 } \\
\text { predicted }\end{array}$ & XM_001067522 & -1.3 \\
\hline EGF, latrophilin and 7 transmembrane domain containing 1 & Eltd1 & NM_022294 & -1.3 \\
\hline Membrane-spanning 4-domains, subfamily A, member 7 & Ms4a7 & XM_215200 & -1.3 \\
\hline RAC/CDC42 exchange factor & Geft & NM_199395 & -1.3 \\
\hline
\end{tabular}




\begin{tabular}{|c|c|c|c|}
\hline Gene name & $\begin{array}{l}\text { Gene } \\
\text { symbol }\end{array}$ & $\begin{array}{l}\text { Accession } \\
\text { number }\end{array}$ & $\begin{array}{l}\text { Fold } \\
\text { change }\end{array}$ \\
\hline Dickkopf homolog 3 (Xenopus laevis) & $D k k 3$ & NM_138519 & -1.3 \\
\hline WNT1 inducible signaling pathway protein 1 & Wisp1 & NM_031716 & -1.3 \\
\hline \multicolumn{4}{|l|}{ Transport } \\
\hline Chloride channel calcium activated 3 & Clca3 & XM_217689 & 1.4 \\
\hline Solute carrier family 15 (oligopeptide transporter), member 1 & Slc15a1 & NM_057121 & 1.4 \\
\hline Solute carrier family 4 , member 4 & Slc4a4 & NM_053424 & 1.4 \\
\hline Chloride intracellular channel 5 & Clic5 & NM_053603 & 1.3 \\
\hline Fatty acid binding protein 4 , adipocyte & Fabp 4 & NM_053365 & -1.5 \\
\hline $\begin{array}{l}\text { Solute carrier family } 11 \text { (proton-coupled divalent metal ion } \\
\text { transporters), member } 1\end{array}$ & Slc11a1 & NM_001031658 & -1.5 \\
\hline Potassium inwardly-rectifying channel, subfamily J, member 8 & Kcnj8 & NM_017099 & -1.4 \\
\hline Guanylate cyclase activator $2 \mathrm{~b}$ & Guca2b & NM_022284 & -1.3 \\
\hline $\begin{array}{l}\text { Solute carrier family } 28 \text { (sodium-coupled nucleoside } \\
\text { transporter), member } 2\end{array}$ & Slc28a2 & NM_031664 & -1.3 \\
\hline Lipoprotein lipase & Lpl & NM_012598 & -1.3 \\
\hline \multicolumn{4}{|l|}{ Cytoskeleton } \\
\hline Dynein cytoplasmic 1 intermediate chain 1 & Dync1i1 & NM_019234 & 1.3 \\
\hline Myosin, heavy polypeptide 10 , non-muscle & Myh10 & NM_031520 & -1.7 \\
\hline Keratin complex 2 , basic, gene 7 & Krt2-7 & XM_217035 & -1.5 \\
\hline Similar to tensin & LOC301509 & XM_237286 & -1.4 \\
\hline Tubulin, beta 6 & Tubb6 & NM_001025675 & -1.4 \\
\hline Dnein cytoplasmic 2 light intermediate chain 1 & Dync2li1 & NM_001013940 & -1.3 \\
\hline Similar to tropomyosin 1, embryonic fibroblast - rat & MGC109519 & NM_001024345 & -1.3 \\
\hline \multicolumn{4}{|l|}{ Growth factors } \\
\hline Insulin-like growth factor binding protein 3 & Igfbp3 & NM_012588 & -1.5 \\
\hline cDNA clone IMAGE: 7110383 & Igfbp5 & BC087030 & -1.5 \\
\hline Platelet derived growth factor receptor, beta polypeptide & Pdgfrb & NM_031525 & -1.4 \\
\hline Platelet derived growth factor receptor, alpha polypeptide & Pdgfra & XM_214030 & -1.4 \\
\hline Bone morphogenetic protein 4 & Bmp4 & NM_012827 & -1.4 \\
\hline Insulin-like growth factor binding protein 4 & Igfbp 4 & NM_001004274 & -1.4 \\
\hline Follistatin & Fst & NM_012561 & -1.4 \\
\hline \multicolumn{4}{|l|}{ Hormones } \\
\hline Somatostatin & Sst & NM_012659 & 1.4 \\
\hline Cytidine monophospho-N-acetylneuraminic acid hydroxylase & Cmah & NM_001024273 & 1.3 \\
\hline Natriuretic peptide precursor type B & Nppb & NM_031545 & -2.5 \\
\hline
\end{tabular}




\begin{tabular}{|c|c|c|c|}
\hline Gene name & $\begin{array}{l}\text { Gene } \\
\text { symbol }\end{array}$ & $\begin{array}{l}\text { Accession } \\
\text { number }\end{array}$ & $\begin{array}{l}\text { Fold } \\
\text { change }\end{array}$ \\
\hline Inhibin beta-A & Inhba & NM_017128 & -1.4 \\
\hline Apelin, AGTRL1 ligand & Apln & NM_031612 & -1.4 \\
\hline \multicolumn{4}{|l|}{ Transcription } \\
\hline Basic helix-loop-helix domain containing, class B3 & Bhlhb3 & NM_133303 & -1.6 \\
\hline Paired related homeobox 2 & Prrx2 & XM_238327 & -1.5 \\
\hline Zinc finger, DHHC domain containing 2 & Zdhhc2 & NM_145096 & -1.4 \\
\hline Snail homolog 1 (Drosophila) & Snai1 & NM_053805 & -1.4 \\
\hline \multicolumn{4}{|l|}{ Blood coagulation } \\
\hline Thrombomodulin & Thbd & NM_031771 & -1.5 \\
\hline Coagulation factor III & F3 & NM_013057 & -1.4 \\
\hline Protein $\mathrm{C}$ receptor, endothelial & Procr & NM_001025733 & -1.4 \\
\hline Annexin A5 & Anxa5 & NM_013132 & -1.3 \\
\hline \multicolumn{4}{|l|}{ Prostaglandins } \\
\hline Hydroxyprostaglandin dehydrogenase 15 (NAD) & Hpgd & NM_024390 & 1.3 \\
\hline Prostaglandin E synthase & Ptges & NM_021583 & -1.4 \\
\hline Prostaglandin-endoperoxide synthase 2 & Ptgs2 & NM_017232 & -1.3 \\
\hline \multicolumn{4}{|l|}{ Glutathione } \\
\hline Glutathione S-transferase A3 & Gsta3 & NM_031509 & -1.6 \\
\hline Glutathione-S-transferase, alpha type 2 & Gsta2 & NM_017013 & -1.4 \\
\hline Gamma-glutamyltransferase 1 & Ggt1 & NM_053840 & -1.3 \\
\hline \multicolumn{4}{|l|}{ Defense } \\
\hline Defensin alpha 6 & Defa6 & NM_001033076 & 2.8 \\
\hline Defensin related cryptdin 4 & Defcr 4 & NM_001013053 & 2.4 \\
\hline Lysozyme & Lyz & NM_012771 & -1.5 \\
\hline \multicolumn{4}{|l|}{ Barrier } \\
\hline $\begin{array}{l}\text { O88310 Intelectin (Mus musculus } 10 \text { day old male } \\
\text { pancreas cDNA, RIKEN full-length enriched library, } \\
\text { clone:1810012B21 product:intelectin, full insert } \\
\text { sequence), complete }\end{array}$ & $\begin{array}{l}\text { ENSRNO } \\
\text { T00000 } \\
006196\end{array}$ & TC531555 & 1.4 \\
\hline Trefoil factor 1 & $T f f 1$ & NM_057129 & 1.3 \\
\hline Claudin 9 & Cldn9 & NM_001011889 & -1.5 \\
\hline \multicolumn{4}{|l|}{ Angiogenesis } \\
\hline Endothelin 2 & Edn2 & NM_012549 & 1.4 \\
\hline Endothelin receptor type B & $E d n r b$ & NM_017333 & -1.6 \\
\hline Predicted: angiopoietin 2 & Angpt2 & XM_001065522 & -1.5 \\
\hline
\end{tabular}




\begin{tabular}{|c|c|c|c|}
\hline Gene name & $\begin{array}{l}\text { Gene } \\
\text { symbol }\end{array}$ & $\begin{array}{l}\text { Accession } \\
\text { number }\end{array}$ & $\begin{array}{l}\text { Fold } \\
\text { change }\end{array}$ \\
\hline \multicolumn{4}{|l|}{ Miscellaneous } \\
\hline Serine protease inhibitor, Kazal type 1 & Spink1 & NM_012674 & 1.5 \\
\hline $\begin{array}{l}\text { RVL20684 Wackym-Soares normalized rat vestibular cDNA } \\
\text { library cDNA 5' }\end{array}$ & DV728079 & DV728079 & 1.3 \\
\hline Similar to RIKEN cDNA 4930449E07 (LOC287371) & $X M_{-} 213320$ & XM_213320 & 1.3 \\
\hline Leucine rich repeat containing 48 & $\operatorname{Lrrc48}$ & NM_001013857 & 1.3 \\
\hline Ghrelin precursor & Ghrl & NM_021669 & 1.3 \\
\hline Selenium binding protein 2 & Selenbp1 & NM_080892 & 1.3 \\
\hline Deleted in malignant brain tumors 1 & Dmbt1 & XM_577842 & 1.3 \\
\hline Enolase 3, beta & Eno3 & NM_012949 & 1.3 \\
\hline Predicted: similar to LRRGT00057 & $\begin{array}{l}\text { RGD1565406 } \\
\text { predicted }\end{array}$ & XM_216693 & 1.3 \\
\hline Arginase 1 & $\operatorname{Arg} 1$ & NM_017134 & -2.3 \\
\hline Hydroxysteroid 11-beta dehydrogenase 1 & $H s d 11 b 1$ & NM_017080 & -1.9 \\
\hline Unknown & $\begin{array}{l}\text { ENSRNOTOO } \\
\text { OOOOH7221 }\end{array}$ & & -1.9 \\
\hline Unknown & $\begin{array}{l}\text { ENSRNOTOO } \\
\text { O00043097 }\end{array}$ & & -1.7 \\
\hline $\begin{array}{l}\text { EST349068 Rat gene index, normalized rat, norvegicus, Bento } \\
\text { Soares cDNA clone RGIEH67 5' end }\end{array}$ & $A W 917764$ & AW917764 & -1.7 \\
\hline ATPase, $\mathrm{H}+$ transporting, $\mathrm{V} 0$ subunit $\mathrm{D}$, isoform 2 & Atp $6 v 0 d 2$ & NM_001011972 & -1.6 \\
\hline Cytochrome P450, family 26 , subfamily b, polypeptide 1 & Сур26b1 & NM_181087 & -1.6 \\
\hline Similar to hypothetical protein MGC6835 & LOC500300 & NM_001024334 & -1.6 \\
\hline DEPA0489 Rat Lambda ZAP Express Library cDNA 5' & BE126741 & BE126741 & -1.6 \\
\hline Similar to RIKEN cDNA 1110014F24 (LOC361548) & $\begin{array}{l}\text { RGD156152 } \\
\text { 1_predicted }\end{array}$ & XM_341835 & -1.6 \\
\hline RNU02094 Dahl salt resistant strain clone etb satellite DNA & U02094 & U02094 & -1.6 \\
\hline Similar to mac25 (LOC289560) & $X M_{2} 214014$ & XM_214014 & -1.5 \\
\hline Ly6/Plaur domain containing 3 & Lypd3 & NM_021759 & -1.5 \\
\hline $\begin{array}{l}\text { EST293291 Normalized rat ovary, Bento Soares Rattus sp. } \\
\text { cDNA clone RGIBB31 5' end }\end{array}$ & $A W 142999$ & AW142999 & -1.5 \\
\hline Predicted: CD248 antigen, endosialin & $\begin{array}{l}\text { Cd248 } \\
\text { predicted }\end{array}$ & XM_238151 & -1.5 \\
\hline Schwannomin interacting protein 1 & Schip1 & XM_215570 & -1.5 \\
\hline $\begin{array}{l}\text { RVL5189 Wackym-Soares normalized rat vestibular cDNA } \\
\text { library cDNA 5' }\end{array}$ & DV715514 & DV715514 & -1.5 \\
\hline $\begin{array}{l}\text { BF558825 UI-R-A1-du-g-03-0-UI.r1 UI-R-A1 cDNA clone UI-R- } \\
\text { A1-du-g-03-0-UI 5' }\end{array}$ & BF558825 & BF558825 & -1.5 \\
\hline
\end{tabular}




\begin{tabular}{|c|c|c|c|}
\hline Gene name & $\begin{array}{l}\text { Gene } \\
\text { symbol }\end{array}$ & $\begin{array}{l}\text { Accession } \\
\text { number }\end{array}$ & $\begin{array}{l}\text { Fold } \\
\text { change }\end{array}$ \\
\hline G0/G1 switch gene 2 & G0s2 & NM_001009632 & -1.5 \\
\hline $\begin{array}{l}\text { Predicted: similar to adenomatosis polyposis coli down- } \\
\text { regulated1 }\end{array}$ & LOC682861 & XM_001063452 & -1.5 \\
\hline $\begin{array}{l}\text { EST348049 Rat gene index, normalized rat, norvegicus, Bento } \\
\text { Soares cDNA clone RGIDU28 5' end }\end{array}$ & $A W 916836$ & AW916836 & -1.5 \\
\hline Similar to cDNA sequence BC004044 & RGD1311980 & XM_573367 & -1.5 \\
\hline $\begin{array}{l}\text { Shultzomica } 03441 \text { Rat lung airway and parenchyma cDNA } \\
\text { libraries cDNA clone Contig3039 5' }\end{array}$ & CF110190 & CF110190 & -1.4 \\
\hline Unknown & $\begin{array}{l}A \_44 \_ \\
P 806889\end{array}$ & & -1.4 \\
\hline Similar to RIKEN cDNA 6330442E10 gene & $\begin{array}{l}\text { RGD156262 } \\
\text { 2_predicted }\end{array}$ & XM_578556 & -1.4 \\
\hline Enolase 2, gamma & Eno2 & NM_139325 & -1.4 \\
\hline $\begin{array}{l}\text { EST294836 Rat gene index, normalized rat, norvegicus, Bento } \\
\text { Soares cDNA clone RGIZB87 5' end }\end{array}$ & $A W 914058$ & AW914058 & -1.4 \\
\hline $\begin{array}{l}\text { UI-R-E0-bz-f-05-0-UI.s2 UI-R-E0 cDNA clone UI-R-E0-bz-f-05- } \\
\text { 0-UI 3' similar to gb }\end{array}$ & AA860038 & AA860038 & -1.4 \\
\hline Unknown & $\begin{array}{l}\text { A_44_ } \\
P 950925\end{array}$ & & -1.4 \\
\hline Similar to hypothetical protein FLJ20898 & MGC105601 & NM_001009620 & -1.4 \\
\hline WAP four-disulfide core domain 1 & $W f d c 1$ & NM_133581 & -1.4 \\
\hline $\begin{array}{l}\text { EST291990 Normalized rat ovary, Bento Soares Rattus sp. } \\
\text { cDNA clone RGICN24 5' end similar to metalloproteinase-3 } \\
\text { tissue inhibitor TIMP-3 }\end{array}$ & $A W 141875$ & AW141875 & -1.4 \\
\hline $\begin{array}{l}\text { UI-R-C2-nm-a-01-0-UI.s1 UI-R-C2 cDNA clone UI-R-C2-nm-a- } \\
\text { 01-0-UI 3' }\end{array}$ & AI072466 & AI072466 & -1.4 \\
\hline Septin 4 & Sept4 & NM_001011893 & -1.4 \\
\hline Q7TPJ2 Ac2-224, partial (16\%) & TC523657 & TC523657 & -1.4 \\
\hline $\begin{array}{l}\text { EST346960 Normalized rat embryo, Bento Soares Rattus sp. } \\
\text { cDNA clone RGICZ05 5' end }\end{array}$ & $A W 915656$ & AW915656 & -1.4 \\
\hline Q9ERL1 NADPH oxidase beta subunit gp91phox, complete & TC551872 & TC551872 & -1.4 \\
\hline Annexin A3 & Аnха3 & NM_012823 & -1.4 \\
\hline $\begin{array}{l}\text { UI-R-C2p-qq-e-09-0-UI.r1 UI-R-C2p cDNA clone UI-R-C2p-qq- } \\
\text { e-09-0-UI 5' }\end{array}$ & BF544728 & BF544728 & -1.4 \\
\hline Q7ZXT5 Alg5-prov protein, partial (6\%) & TC546120 & TC546120 & -1.4 \\
\hline Unknown & TC535812 & & -1.3 \\
\hline Sorting nexin 10 & Snx10 & NM_001013085 & -1.3 \\
\hline Predicted: similar to scavenger receptor type A SR-A & $\begin{array}{l}\text { RGD156431 } \\
\text { 6_predicted }\end{array}$ & XM_573919 & -1.3 \\
\hline
\end{tabular}




\begin{tabular}{|c|c|c|c|}
\hline Gene name & $\begin{array}{l}\text { Gene } \\
\text { symbol }\end{array}$ & $\begin{array}{l}\text { Accession } \\
\text { number }\end{array}$ & $\begin{array}{l}\text { Fold } \\
\text { change }\end{array}$ \\
\hline Argininosuccinate synthetase & Ass & NM_013157 & -1.3 \\
\hline Ficolin B & Fcnb & NM_053634 & -1.3 \\
\hline $\begin{array}{l}\text { Predicted: similar to RNA binding protein gene with multiple } \\
\text { splicing }\end{array}$ & $\begin{array}{l}\text { RGD156106 } \\
\text { 7_predicted }\end{array}$ & XM_573923 & -1.3 \\
\hline $\begin{array}{l}\text { UI-R-C3-so-h-10-0-UI.r1 UI-R-C3 cDNA clone UI-R-C3-so-h- } \\
\text { 10-0-UI 5' }\end{array}$ & BF542741 & BF542741 & -1.3 \\
\hline Unknown & TC533989 & & -1.3 \\
\hline Similar to Antxr2 protein & LOC305633 & XM_223745 & -1.3 \\
\hline Cytochrome b-245, beta polypeptide & $C y b b$ & NM_023965 & -1.3 \\
\hline Protocadherin 7 & Pcdh7 & NM_001004087 & -1.3 \\
\hline Similar to cysteine and tyrosine-rich protein 1 & LOC304138 & NM_001013980 & -1.3 \\
\hline PE responsive protein c64 & Perc64 & XM_001053103 & -1.3 \\
\hline $\begin{array}{l}\text { Predicted: similar to ABI gene family, member } 3 \text { (NESH) } \\
\text { binding protein }\end{array}$ & $\begin{array}{l}\text { RGD156271 } \\
\text { 7_predicted }\end{array}$ & XM_344015 & -1.3 \\
\hline Predicted: sushi-repeat-containing protein, X-linked 2 & $\begin{array}{l}\text { Srpx } 2_{-} \\
\text {predicted }\end{array}$ & XM_228444 & -1.3 \\
\hline $\begin{array}{l}\text { EST349691 Rat gene index, normalized rat, norvegicus, Bento } \\
\text { Soares cDNA clone RGIER58 5' end }\end{array}$ & AW918387 & AW918387 & -1.3 \\
\hline $\begin{array}{l}\text { EST293223 Normalized rat ovary, Bento Soares Rattus sp. } \\
\text { cDNA clone RGIAZ71 5' end }\end{array}$ & AW142932 & AW142932 & -1.3 \\
\hline Similar to hypothetical protein MGC33926 & MGC94782 & NM_001004282 & -1.3 \\
\hline Predicted: similar to RIKEN cDNA 1600013K19 & $\begin{array}{l}\text { RGD130774 } \\
\text { 9_predicted }\end{array}$ & XM_238473 & -1.3 \\
\hline G protein-coupled receptor 176 & Gpr176 & XM_342493 & -1.3 \\
\hline Germ cell associated 1 & Gsg1 & NM_001013166 & -1.3 \\
\hline Predicted: similar to Cd300D antigen & LOC498022 & XM_213514 & -1.3 \\
\hline Predicted: prickle-like 1 (Drosophila), transcript variant 2 & Prickle1 & XM_001057488 & -1.3 \\
\hline Similar to Antxr2 protein & LOC305633 & XM_223745 & -1.3 \\
\hline Cytochrome b-245, beta polypeptide & $C y b b$ & NM_023965 & -1.3 \\
\hline Protocadherin 7 & Pcdh7 & NM_001004087 & -1.3 \\
\hline Similar to cysteine and tyrosine-rich protein 1 & LOC304138 & NM_001013980 & -1.3 \\
\hline PE responsive protein c64 & Perc64 & XM_001053103 & -1.3 \\
\hline $\begin{array}{l}\text { Predicted: similar to ABI gene family, member } 3 \text { (NESH) } \\
\text { binding protein }\end{array}$ & $\begin{array}{l}\text { RGD156271 } \\
\text { 7_predicted }\end{array}$ & XM_344015 & -1.3 \\
\hline Predicted: sushi-repeat-containing protein, X-linked 2 & $\begin{array}{l}\text { Srpx2 } \\
\text { predicted }\end{array}$ & XM_228444 & -1.3 \\
\hline $\begin{array}{l}\text { EST349691 Rat gene index, normalized rat, norvegicus, Bento } \\
\text { Soares cDNA clone RGIER58 5' end }\end{array}$ & AW918387 & AW918387 & -1.3 \\
\hline
\end{tabular}




\begin{tabular}{lllc}
\hline $\begin{array}{l}\text { Gene name } \\
\begin{array}{l}\text { EST293223 Normalized rat ovary, Bento Soares Rattus sp. } \\
\text { cDNA clone RGIAZ71 5' end }\end{array}\end{array}$ & $\begin{array}{l}\text { Gene } \\
\text { symbol }\end{array}$ & $\begin{array}{l}\text { Accession } \\
\text { number }\end{array}$ & $\begin{array}{l}\text { Fold } \\
\text { change }\end{array}$ \\
$\begin{array}{l}\text { Similar to hypothetical protein MGC33926 } \\
\text { Predicted: similar to RIKEN cDNA 1600013K19 }\end{array}$ & AW142932 & -1.3 \\
& MGC94782 & NM_001004282 & -1.3 \\
G protein-coupled receptor 176 & $\begin{array}{l}\text { RGD130774 } \\
\text { 9_predicted }\end{array}$ & XM_238473 & -1.3 \\
$\begin{array}{l}\text { Germ cell associated 1 } \\
\text { Predicted: similar to Cd300D antigen }\end{array}$ & Gpr176 & XM_342493 & -1.3 \\
Predicted: prickle-like 1 (Drosophila), transcript variant 2 & Grick1 & NM_001013166 & -1.3 \\
\hline
\end{tabular}

Results are genes classified in different processes that are differentially regulated at least 1.3-fold with a p-value $<0.05$ (Students $t$ test) in colonic mucosa of transgenic rats fed the calcium diet ( $n=8)$ compared to the transgenic rats fed the control diet $(n=9)$. Fold changes $<1$ are reported as their negative inverse. Genes are sorted based on absolute fold change of control. Only processes with at least 3 regulated genes are mentioned.
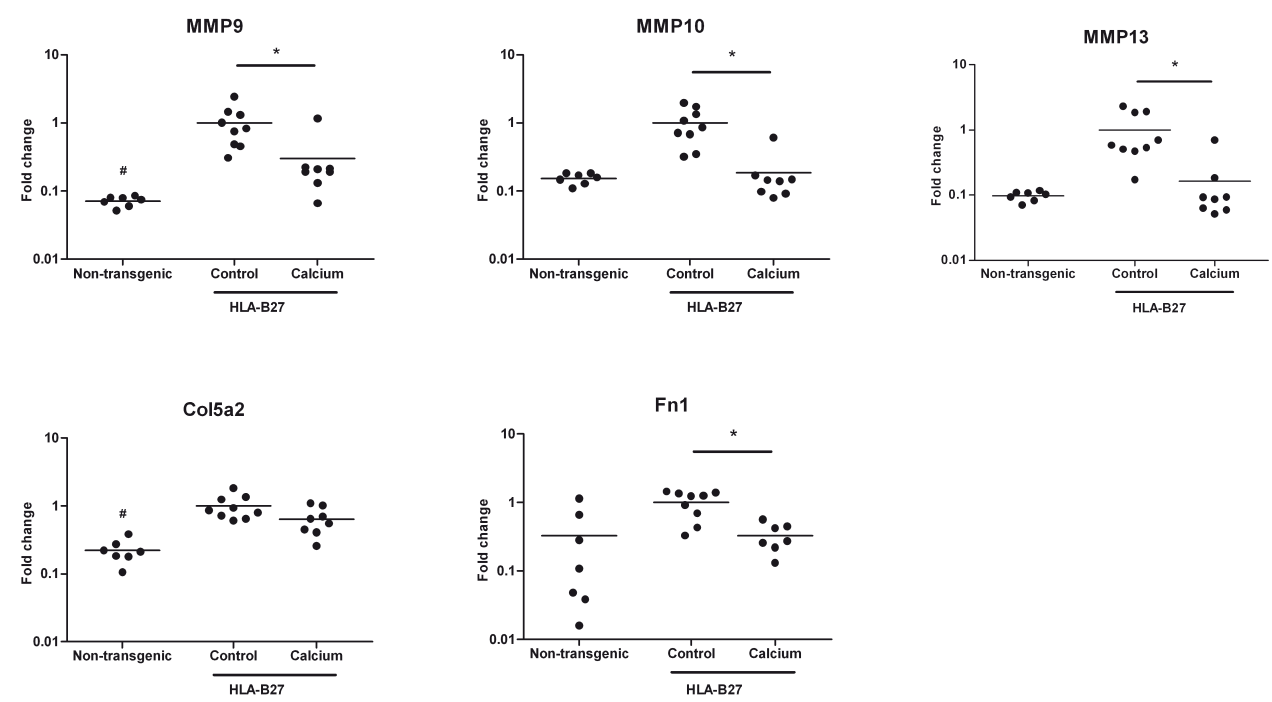

Supplemental figure 1: Effect of dietary calcium on individual colonic mucosal mRNA expression of MMP9, MMP10, MMP13, Col5a2 and Fn1 in HLA-B27 transgenic rats as analyzed by Q-PCR. The mean expression of transgenic rats on the control diet is set at 1. The mean of each group is indicated by a line (non-transgenic rats: $n=7$; control transgenic rats: $n=9$; calcium-fed transgenic rats: $n=8$ ). Aldolase, ADP-ribosylation factor 1 and $\beta$-actin were used as reference genes for normalization of the data. ${ }^{*}$ Different from control transgenic rats, $P<0.05$. "Different from transgenic rats, $P<0.05$. 


\title{
Supplemental antioxidants, in contrast to calcium, do not ameliorate colitis development in HLA-B27 transgenic rats despite extremely low glutathione levels in colonic mucosa
}

\author{
Marloes A.A. Schepens \\ Carolien Vink \\ Arjan J. Schonewille \\ Hennie M.J. Roelofs \\ Robert-Jan Brummer \\ Roelof van der Meer \\ Ingeborg M.J. Bovee-Oudenhoven
}

Inflammatory Bowel Diseases. 2010 Dec 22. In press (slightly modified version) 


\section{ABSTRACT}

Objective: Oxidative stress is presumed to play an important role in inflammatory bowel disease (IBD). Accordingly, antioxidant supplementation might be protective. Dietary calcium inhibited colitis development in HLA-B27 transgenic rats, an animal model mimicking IBD. As antioxidants might act at mucosa level and calcium predominantly in the gut lumen, we hypothesize that the combination has additive protective effects on colitis development.

Methods: HLA-B27 rats were fed a control diet, or the same diet supplemented with the antioxidants glutathione, vitamin $C$ and vitamin $E$, or supplemented with both antioxidants and calcium. Oxidative stress in colonic mucosa, colonic inflammation, intestinal permeability and diarrhea were quantified.

Results: Intestinal permeability, diarrhea, myeloperoxidase and interleukin- $1 \beta$ levels were significantly lower in rats fed both antioxidants and calcium compared to rats supplemented with antioxidants only. No beneficial effects were observed in rats fed the diet supplemented with antioxidants only. Strikingly, despite extremely low colonic mucosal glutathione levels in HLA-B27 rats, there was no oxidative stress-related damage. Subsequent analyses showed no defect in expression of glutathione synthesis genes. Additional experiments, comparing young and older HLA-B27 rats, showed that glutathione levels and also reactive oxygen species production decreased with progression of intestinal inflammation.

Conclusion: Antioxidant supplementation was ineffective in HLA-B27 rats despite low mucosal glutathione levels, because colitis development did not coincide with oxidative stress in this model. This indicates that the neutrophilic respiratory burst, and thus innate immune defense, is compromised in HLB-B27 rats. As supplementation with both calcium and antioxidants attenuated colitis development, this protective effect is contributed to calcium only. 


\section{INTRODUCTION}

Crohn's disease and ulcerative colitis, two forms of inflammatory bowel disease (IBD), are characterized by chronic intestinal inflammation, resulting from mucosal barrier dysfunction, altered microbial factors and dysregulated immune responses ${ }^{(1)}$. Despite a genetic influence, these different determinants of the pathogenesis of IBD can be modulated by environmental factors and dietary components in particular. Therefore, nutrition might be an interesting preventive option for IBD patients as well as a supporting therapeutic strategy ${ }^{(2)}$.

The inflamed bowel of IBD patients is infiltrated with leukocytes, generating large amounts of reactive oxygen species (ROS), presumed to result in oxidative stress and subsequent mucosal damage ${ }^{(3)}$. Antioxidants are proposed to prevent oxidative damage in general ${ }^{(2,4)}$. Numerous antioxidants exist and they all have specific roles in the mucosal antioxidant defense system. Therefore, for protection against oxidative stress, it might be more effective in nutritional interventions to supplement a mixture of antioxidants, each having different chemical properties, than just one antioxidant $t^{(5)}$.

In this dietary intervention study, we chose to supplement with vitamin C, vitamin $\mathrm{E}$ and glutathione, being central players in the antioxidant network. Vitamin $\mathrm{C}$ is a water soluble antioxidant, vitamin E a lipid soluble antioxidant, while glutathione is one of the major intracellular antioxidants ${ }^{(6)}$. Moreover, the bioactivity of each of these three antioxidants is strongly dependent on the others. For example, vitamin E can be directly regenerated from vitamin $\mathrm{E}$ radical by vitamin $\mathrm{C}^{(7)}$, and in case of glutathione deficiency vitamin $C$ can spare glutathione ${ }^{(8-10)}$. Although literature on oral glutathione supplementation is contradictory, orally supplied glutathione has been shown to increase intestinal mucosal glutathione levels, also in situations of suppressed de novo glutathione synthesis ${ }^{(11-13)}$.

We have previously shown that dietary calcium inhibits colitis development in HLA-B27 transgenic rats ${ }^{(14)}$. The HLA-B27 transgenic rat is a well-characterized animal model of chronic intestinal inflammation. Rats overexpressing the human HLA-B27/ $\beta 2$ microglobulin gene spontaneously develop an inflammatory disease mainly involving the gastrointestinal tract and the colon in particular ${ }^{(15)}$. Also in humans, the HLA-B27 gene is associated with inflammatory disorders ${ }^{(16)}$. Although the exact underlying mechanism has not been characterized yet, it has been shown that HLA-B27-expressing innate immune cells have an essential role in determining mucosal immune responses to commensal bacteria in the development of colitis in the transgenic rats ${ }^{(17)}$. Additionally, we have shown in several controlled rat and human studies that dietary calcium improves intestinal resistance against infections with foodborne bacterial pathogens and that it strengthens the mucosal barrier ${ }^{(18-20)}$. It is hypothesized that calcium exerts this protection in part by precipitating irritating bile acids and fatty acids, thereby reducing cytotoxicity of the fecal stream. The decrease in luminal cytotoxicity reduces damage to intestinal epithelial cells and thus reinforces mucosal integrity ${ }^{(21-22)}$. We speculated that dietary antioxidants, suggested to be active in the intestinal mucosa, and calcium, appreciated for its luminal effects, have an additive protective potential 
against colonic inflammation.

In the present study we investigated the effect of a mixture of supplemental antioxidants with or without extra calcium on colitis development in HLA-B27 transgenic rats. It is hypothesized that a mixture of vitamin $\mathrm{C}$, vitamin $\mathrm{E}$ and glutathione reduces the severity of colonic inflammation but that the combination of dietary antioxidants plus calcium has superior efficacy. As remarkably low glutathione levels were observed in colonic mucosa of HLA-B27 rats, we performed an additional experiment. Young and older transgenic rats were compared to determine possible causality of low glutathione levels to colitis development. Moreover, since glutathione levels were low and oxidative stressrelated damage seemed to be absent in the HLA-B27 rats in the dietary intervention study, we studied the mucosal and systemic ROS production of the transgenic rats.

\section{MATERIAL AND METHODS}

\section{Experimental design of dietary intervention study: animals and diets}

The experimental protocol was approved by the animal welfare committee of Wageningen University (The Netherlands). Female HLA-B27/ $\beta 2$-microglobulin transgenic rats on an inbred Fisher 344 background ( $n=9$ per dietary group) and their non-transgenic counterparts ( $n=7$; Taconic Farms, Inc, Germantown, NY), 8-10 weeks old with a mean body weight of $140 \mathrm{~g}$ at the start of the experiment, were housed individually in metabolic cages. Animals were kept in a temperature- and humidity-controlled environment during a 12-h light-dark cycle. Transgenic rats were fed a purified 'humanized' Westerntype diet ad libitum which contained in the control situation (per kg diet): $200 \mathrm{~g}$ acid casein, 326 g corn starch, 174 g glucose, 160 g palm oil, 40 g corn oil, 50 g cellulose, 2 g chromium EDTA (CrEDTA; see below) and 5.16 g CaHPO $42 \mathrm{H}_{2} \mathrm{O}$ (corresponding to 30 $\mathrm{mmol}$ calcium/kg diet; Sigma-Aldrich, St Louis, MO). Vitamins and minerals (other than calcium) were added to the diets according to $\operatorname{AIN93}^{(23)}$. To better mimic the composition of a Western human diet, diets were relatively low in calcium and had a high fat content in comparison with AIN93 recommendations for rodent diets. The experimental diets were supplemented with the antioxidants glutathione $(25 \mathrm{mmol} / \mathrm{kg}$ diet $)$, vitamin $\mathrm{C}$ (2 $\mathrm{g} / \mathrm{kg}$ diet) and vitamin $\mathrm{E}$ ( $\alpha$-tocopherol acetate; $0.68 \mathrm{~g} / \mathrm{kg}$ diet extra), or with both these antioxidants and calcium ( $90 \mathrm{mmol} / \mathrm{kg}$ diet extra) (all supplements: Sigma-Aldrich), all at the expense of glucose. Because of the limited availability of transgenic rats, we could not include a dietary group supplemented with calcium only, but the effect of solely calcium has been reported recently ${ }^{(14)}$. To prevent possible degradation of the antioxidants by light or air, feed was divided into daily portions per dietary group, vacuum packed and stored at $4^{\circ} \mathrm{C}$ until use. In addition, food was supplied daily to the rats just before dark (eating period). The non-transgenic rats were fed the control diet. Inert CrEDTA was added to the diets to quantify intestinal permeability ${ }^{(24)}$. CrEDTA solution was prepared as described elsewhere and subsequently freeze-dried ${ }^{(25)}$. To check complete formation of the CrEDTA complex, the prepared CrEDTA solution was passed through a cation-exchange resin column (Chelex 100 Resin; Bio-Rad, Hercules, $\mathrm{CA}$ ) and no uncomplexed $\mathrm{Cr}^{3+}$ ions were present. Food intake was recorded daily and 
animal weight twice every week. Time of manifestation of colitis in this animal model is variable between different studies and therefore difficult to predict in advance. To follow up, intestinal permeability and diarrhea were monitored every one or two weeks. Nine weeks after the start of the dietary intervention, when a clear increase in intestinal permeability and diarrhea was measurable in at least one dietary group (see results), rats were anesthetized with isoflurane. The colon was taken out, and its weight and length were measured. A $1 \mathrm{~cm}$ piece from the middle of the colon was excised and stored in 10\% neutral buffered formalin for histology. The remaining colon parts were longitudinally excised and washed in saline to remove residual luminal contents. Subsequently, the mucosa was scraped off using a spatula and immediately snap-frozen in liquid nitrogen until further processing and analyses.

\section{Colonic histology}

Colonic tissue specimens were embedded in paraffin. Slices were stained with hematoxylin and eosin. Before analysis, slides were recoded to guarantee blind scoring. A validated histological scoring system for inflammation was used. Scores ranged from 0 to 4 based on criteria such as inflammatory cell infiltration, depletion of goblet cells, mucosa thickening and destruction of mucosal architecture ${ }^{(26)}$.

\section{Measurement of intestinal permeability and diarrhea}

Total $24 \mathrm{~h}$ urine samples were collected on one day every week. For CrEDTA measurement, urine was mixed 1:1 with $50 \mathrm{~g} / \mathrm{L}$ trichloroacetic acid and centrifuged for 2 $\min$ at $14,000 \mathrm{~g}$. After dilution of the supernatants with $0.5 \mathrm{~g} / \mathrm{L} \mathrm{CsCl}$ solution, chromium was analyzed by inductively coupled plasma-atomic emission spectrophotometry (ICPAES; Varian, Mulgrave, Australia). Total 3x24 h feces was collected every two weeks and freeze-dried. Percentage fecal wet weight was determined to quantify diarrhea.

\section{Myeloperoxidase (MPO) and interleukin-1 $\beta$ (IL-1 $\beta$ ) analysis in colonic mucosa}

Frozen colonic mucosal scrapings were manually crushed in liquid nitrogen using a pre-cooled mortar and pestle. After homogenization, part of the powder was used for RNA isolation (see below), and the remaining portion was suspended in a solution containing $200 \mathrm{mM}$ sucrose, $20 \mathrm{mM}$ Tris (pH 7.4), $1 \mathrm{mM}$ dithiothreitol and protease inhibitors before protein analyses. In these samples, MPO activity was measured using a colorimetric assay as described by Grisham et $\mathrm{al}^{(27)}$. MPO activity was expressed as units per mg protein, using purified MPO (Calbiochem, Darmstadt, Germany) as a standard. IL-1 $\beta$ was determined using an ELISA kit (Biosource, Camarillo, CA) according to the manufacturer's instructions. Total protein content of the scrapings was measured spectrophotometrically using the BCA protein assay kit (Uptima, Montluçon, France) with bovine serum albumin (BSA; Sigma-Aldrich) as standard.

\section{Measurement of oxidative damage in colonic mucosa}

Lipid peroxidation generates malondialdehyde (MDA), which reacts with thiobarbituric acid. By measuring the amount of thiobarbiturate reactive substances (TBARS) in colonic mucosa, lipid peroxidation can be determined, as previously described by 
Ohkawa ${ }^{(28)}$. The amount of mucosal TBARS was expressed as $\mu$ mol MDA/mg protein. Protein carbonyls were analyzed as a marker of oxidative protein damage in colonic mucosa by ELISA as described ${ }^{(29)}$. The concentration of carbonyls was expressed as $\mathrm{nmol} / \mathrm{mg}$ protein.

\section{Analysis of antioxidants in colonic mucosa}

The non-thiol antioxidant capacity was measured in colonic mucosa by using the ferric reducing ability assay (FRAP) as described by Benzie and Strain ${ }^{(30)}$. In the presence of antioxidants, ferric ions are reduced to ferrous ions, leading to formation of a colored ferrous-2,4,6-tripyridyl-s-triazine complex which can be measured spectrophotometrically. Values were expressed as nmol $\mathrm{Fe}^{2+} / \mathrm{mg}$ protein. Total glutathione and cysteine were measured using a slight modification of the assay described by Mansoor et $\mathrm{al}^{(31)}$. This method determines total (reduced, oxidized, and protein-bound forms) glutathione and cysteine by HPLC. Compared to the original method, twice the sample volumes and reagents were used. Briefly, samples of 25 $\mu \mathrm{l}$ were injected into a $150 \times 4.6 \mathrm{~mm}, 3 \mu \mathrm{m}$ PLRP-S column, equipped with a PLRP-S guard column (Polymer Laboratories, Amherst, MA). Flow rate was $1 \mathrm{ml} / \mathrm{min}$ at $30^{\circ} \mathrm{C}$ with elution solvent A (0.1\% trifluoroacetic acid (TFA), 5\% acetonitrile) and solvent B $(0.1 \%$ TFA, $80 \%$ acetonitrile), both diluted with distilled water. The elution profile was as follows: $0-20 \mathrm{~min}, 0 \% \mathrm{~B} ; 20-25 \mathrm{~min}, 16 \% \mathrm{~B} ; 25-30 \mathrm{~min}, 50 \% \mathrm{~B}$, with retention time of bimane derivatives of glutathione of $23 \mathrm{~min}$ and cysteine of $13 \mathrm{~min}$. By using a Spectra Systems FL2000 fluorometer (Spectra Physics, Mountain View, CA) excitation and emission were at 394 and $480 \mathrm{~nm}$, respectively. Plotting and integration of peaks were performed by Chromeleon software 6.6 (Dionex, Sunnyvale, CA). Total glutathione and cysteine results are expressed as $\mu \mathrm{mol} / \mathrm{g}$ protein.

\section{Quantitative real-time PCR (Q-PCR) analysis of mucosal samples}

Total RNA was isolated from frozen homogenized colonic scrapings using TRIzol reagent (Invitrogen, Carlsbad, CA) according to the manufacturer's protocol. RNA was purified with RNeasy columns (Qiagen, Venlo, The Netherlands), and purity and concentration were quantified using the Nanodrop ND-1000 (Isogen Life Science, Maarssen, The Netherlands). Subsequently, $1 \mu \mathrm{g}$ of RNA was reverse-transcribed to obtain complementary DNA (cDNA) using reverse transcriptase and oligo d(T) ${ }_{16}$ primer (Applied Biosystems, Foster City, CA) at $48^{\circ} \mathrm{C}$ for $30 \mathrm{~min}$, followed by $5 \mathrm{~min}$ at $95^{\circ} \mathrm{C}$. Differential expression of individual genes was assessed by Q-PCR, using primers presented in table 1 . For each reaction, $2 \mu \mathrm{l}$ of 10 -fold diluted cDNA was added to $23 \mu \mathrm{l}$ premix, containing $1 \mu \mathrm{l}$ forward primer $(10 \mu \mathrm{mol} / \mathrm{L}), 1 \mu \mathrm{l}$ reverse primer $(10$ $\mu \mathrm{mol} / \mathrm{L}), 8.5 \mu \mathrm{l}$ RNase-free water and $12.5 \mu \mathrm{l}$ Power SYBR green PCR Master Mix (Applied Biosystems). The samples were incubated for $10 \mathrm{~min}$ at $95^{\circ} \mathrm{C}$, followed by 40 amplification cycles. Each cycle consisted of $15 \mathrm{~s}$ at $95^{\circ} \mathrm{C}$ and $1 \mathrm{~min}$ at $60^{\circ} \mathrm{C}$, using the 7500 Fast Real-Time PCR System (Applied Biosystems). The run was completed with melting curve analysis for every PCR product to check for single product formation. A standard curve for each particular gene was run with every assay using serial dilutions of a reference sample (cDNA synthesized from a pooled RNA sample). mRNA levels 
Table 1: Primer information

\begin{tabular}{llll}
\hline Gene & Acc $n r$ & Forward primer $\left(5^{\prime} \rightarrow 3^{\prime}\right)$ & Reverse primer $\left(5^{\prime} \rightarrow 3^{\prime}\right)$ \\
\hline Aldolase & NM_012495 & ATGCCCCACCCATACCCAGCACT & AGCAGCAGTTGGCGGTAGAAGCG \\
Arf1 & NM_022518 & CTCCGAGATGCCGTTCTCTTG & GGTAGCCTGAATGTACCAGTTCC \\
$\gamma$ GCSc & NM_012815 & TTACCGAGGCTACGTGTCAGAC & TGTCGATGGTCAGGTCGATGTC \\
$\gamma$ GCSm & NM_017305 & ACCGGGAACCTGCTCAACTG & GATTTGGGATCTCCATTCATTCAAG \\
GSS & NM_012962 & CGGAGCTGGGTATTTTTGGAG & TGCATGTTCAATGGCTTTGGT \\
xCT & NM_001107673 & CCTGGCATTTGGACGCTACAT & TCAGAATTGCTGTGAGCTTGCA \\
\hline
\end{tabular}

Arf1,ADP-ribosylationfactor $1 ; \gamma G C S c, \gamma$-glutamylcysteine synthetase catalyticsubunit; $\gamma G C S m, \gamma$-glutamylcysteine synthetase modifier subunit; GSS, glutathione synthetase; xCT, cystine transporter of system xc-.

were calculated from the appropriate standard curve, and these data were normalized against the housekeeping genes aldolase and ADP-ribosylation factor 1 as indicated.

\section{Experimental design of study with young and older rats: animals and diets}

The experimental protocol was approved by the animal welfare committee of Wageningen University (The Netherlands). As in the nutritional study, HLA-B27 transgenic rats $(n=17)$ and their non-transgenic counterparts $(n=17)$ were used. The transgenic rats were 9-13 weeks old with a mean body weight of $170 \mathrm{~g}$ at the start of the experiment. The animals were housed and fed the control diet as described above. The 8 youngest transgenic and 8 non-transgenic rats were killed directly at the beginning of the experiment $(t=0)$, while the rest of the rats were monitored until they developed colitis, as explained above for the nutritional study. After 10 weeks ( $t=10)$, the older rats were killed. Orbital blood was collected under anesthesia for measurement of ROS production (see below). During the dissections, the colon was taken out, longitudinally excised and washed in saline. Then, the mucosa was scraped off. Part of this mucosal scraping was immediately snap-frozen for analysis of glutathione, cysteine and MPO as described above. The other part was placed in ice-cold pre-oxygenated PBS for ex vivo analysis of ROS production (see below). In addition, part of the liver was excised and frozen in liquid nitrogen for the measurement of glutathione and cysteine after being homogenized in the same sucrose solution as used for the colonic scrapings (see above).

\section{Measurement of ROS production}

Oxygen radical production was determined by luminol-enhanced chemiluminescence, measured in an automated LB96V Microlumat Plus Luminometer (EG \& G Berthold, Bad Wildberg, Germany) as described ${ }^{(32)}$. Briefly, luminol reacts with ROS, which causes light emission (chemiluminescence). For the ROS production in blood, $200 \mu$ l of diluted blood (1:100 diluted in Hank's Balanced Salt Solution (HBSS)) was added per well in a microplate together with $20 \mu \mathrm{l}$ luminol $(0.5 \mathrm{mM}$ luminol HBSS $/ 0.5 \% \mathrm{BSA})$ and $20 \mu \mathrm{l}$ serum treated zymosan (human serum incubated with zymosan, $10 \mathrm{mg} / \mathrm{ml}$ ) to activate 
phagocytes. For determination of the ROS production in colonic mucosa, the scrapings were cut into smaller pieces which were added to $200 \mu \mathrm{l} \mathrm{PBS/tri-ethylamine} \mathrm{per} \mathrm{well,}$ after which $20 \mu \mathrm{l}$ luminol was added. Chemiluminescence was then monitored every $\min$ for $60 \mathrm{~min}$ at $37^{\circ} \mathrm{C}$. Data were analyzed using Winglow software (EG \& G Berthold). One part of every blood sample was used for neutrophil counting by microscopy. Mean chemiluminescence (area under the curve) was expressed in relative light units (RLU) per neutrophil for the blood measurements, and in RLU/mg protein for the mucosal measurements.

\section{Statistical analysis}

All results are expressed as mean \pm SEM. The two predefined main comparisons of interest for the nutritional study were HLA-B27 rats on the control diet versus HLA-B27 rats on the antioxidants diet, and HLA-B27 rats on the antioxidants diet versus HLA-B27 rats on the diet supplemented with both antioxidants and calcium. Statistics on the dietary effects in transgenic rats were done by using one-way ANOVA or Kruskal-Wallis, depending on normality of the data. If significant, this was followed by Student's test (for normally distributed data) or Mann-Whitney U test (for non-normally distributed data) to identify the significant dietary effects. Non-transgenic rats were included in this study as a non-colitic reference to provide some general comparative information on baseline values of the measured parameters. Accordingly, statistics were done on non-transgenic rats (fed the control diet) versus the transgenic rats on the control diet to determine the inflammatory status as such. Again, Student's t test was applied for normally distributed data and Mann-Whitney U test for non-normally distributed data. The predefined comparisons of interest for the study with young and older rats were young HLA-B27 rats versus older HLA-B27 rats, young non-transgenic rats versus young transgenic rats, and older non-transgenic rats versus older HLA-B27 rats. The latter two comparisons were to check the inflammatory status, while the first comparison was to test the potential aging-effect. Statistics were done as described above for the nutritional study. Differences were considered statistically significant when $\mathrm{p}<0.05$ (all two sided).

\section{RESULTS}

Nutritional intervention: animal growth, food intake and colon length and weight Animal growth $(0.7 \pm 0.04 \mathrm{~g} /$ day $)$ and food intake $(10.4 \pm 0.1 \mathrm{~g} /$ day $)$ of the transgenic rats were not affected by the different diets. Non-transgenic rats had a higher body weight gain $(1.0 \pm 0.1 \mathrm{~g} /$ day; $\mathrm{p}<0.01)$ than transgenic rats despite a similar food intake $(10.2 \pm 0.4 \mathrm{~g} /$ day $)$. Colons of HLA-B27 rats of the control group $(13.1 \pm 0.3 \mathrm{~cm})$ were the same length as the colons of the antioxidants group $(12.7 \pm 0.3 \mathrm{~cm})$, which were shorter than colons of rats fed both extra antioxidants and calcium $(13.6 \pm 0.2 \mathrm{~cm} ; \mathrm{p}<0.05)$. Colon weight of transgenic rats $(2.1 \pm 0.04 \mathrm{~g})$ was much higher than that of nontransgenic rats $(0.9 \pm 0.07 \mathrm{~g} ; \mathrm{p}<0.001)$, which is one of the characteristics of intestinal inflammation. Colon weight was not influenced by the different diets. 


\section{The effect of antioxidants and calcium on intestinal permeability and diarrhea}

Intestinal inflammation is characterized by an increased intestinal permeability and diarrhea. In week 1 of the experiment, intestinal permeability was similar in all groups as measured by urinary CrEDTA excretion. In week 9, a clear increase in intestinal permeability was observed for the HLA-B27 rats on the control diet and the HLA-B27 rats fed the antioxidants diet. In contrast, transgenic rats on the diet with both extra antioxidants and calcium did not develop this colitis-related increase in intestinal permeability when compared to transgenic rats supplemented with antioxidants only (figure $1 ; \mathrm{p}<0.05$ ). A similar protective effect was observed on diarrhea development. Diarrhea was quantified by the measurement of percentage fecal wet weight. Transgenic rats fed both antioxidants and calcium suffered less from diarrhea than transgenic rats fed antioxidants only (figure $2 ; \mathrm{p}<0.05$ ). Intestinal permeability and diarrhea development were similar in HLA-B27 rats fed the diet supplemented with antioxidants only and the transgenic rats fed the control diet.

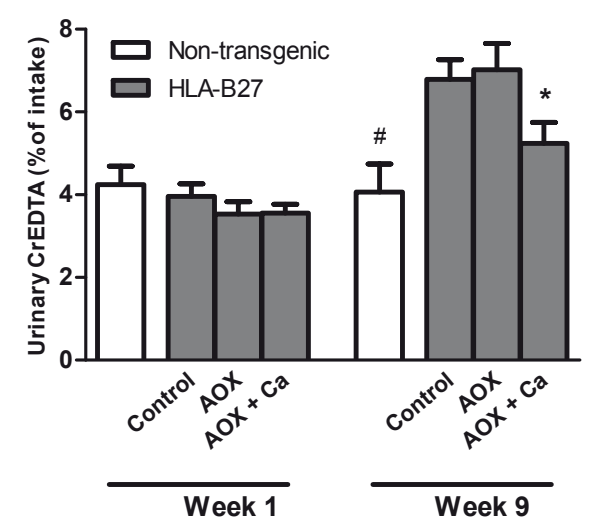

Figure 1: Effect of dietary antioxidants $(A O X)$ and calcium (Ca) on urinary chromium EDTA (CrEDTA) excretion, a marker for intestinal permeability, in HLA-B27 transgenic rats. CrEDTA was added to the diets and analyzed in urine by inductively coupled plasma-atomic emission spectrophotometry. Results are expressed as mean \pm SEM. In week 1 , intestinal permeability was similar for all groups. *Indicates that in week 9 urinary CrEDTA excretion was significantly less increased in transgenic rats fed the diet with both antioxidants and calcium compared to rats fed the diet supplemented with antioxidants only. ${ }^{\#}$ Indicates that non-transgenic rats $(n=7)$ were significantly different from control HLA-B27 rats $(n=9)$.

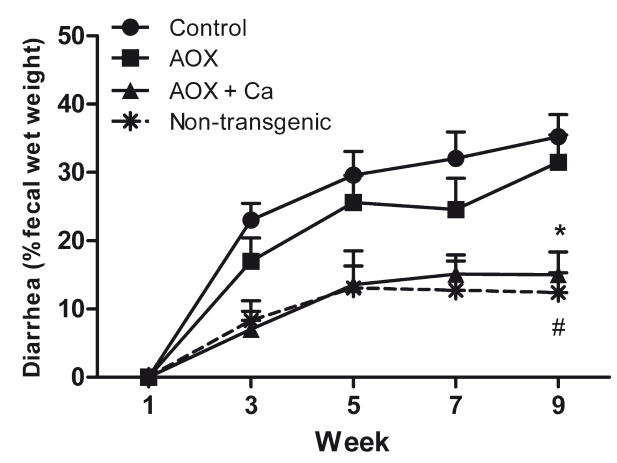

Figure 2: Effect of dietary antioxidants (AOX) and calcium (Ca) on diarrhea development in HLA-B27 transgenic rats. Diarrhea is quantified as the increase of percentage fecal wet weight compared to week 1. Results are expressed as mean \pm SEM. *Indicates that in week 9 transgenic rats fed the diet with both antioxidants and calcium had significantly less diarrhea than transgenic rats supplemented with antioxidants only. "Indicates that non-transgenic rats ( $n=7)$ were significantly different from $H L A-B 27$ rats $(n=9)$, both fed the control diet. 


\section{Antioxidants, calcium and inflammatory parameters}

To quantify inflammation, MPO activity and IL-1 $\beta$ were measured in colonic mucosa. In addition, mucosal inflammation was graded histologically using a semi-quantitative scoring system ${ }^{(26)}$. As expected, the histological inflammation score, mucosal MPO activity and IL-1 $\beta$ concentration were higher for HLA-B27 rats compared to nontransgenic rats. The histological score was significantly different between the dietary groups (Kruskal-Wallis: $\mathrm{p}=0.04$ ). However, when the separate Mann-Whitney U tests were performed, only the comparison between the diet with both antioxidants and calcium and the control diet was significant, and not our predefined comparisons (figure 3A). As observed for the intestinal permeability and diarrhea outcomes, transgenic rats supplemented with both antioxidants and calcium had lower MPO activity and IL-1 $\beta$ levels compared to transgenic rats fed antioxidants only (figure $3 \mathrm{~B}$ and $3 \mathrm{C} ; \mathrm{p}<0.05$ ). Dietary antioxidants alone did not influence the inflammatory parameters compared to the transgenic rats on the control diet.

\section{Oxidative stress-related damage to colonic mucosa}

As dietary antioxidants are proposed to protect against oxidative stress involved in intestinal inflammation, we measured oxidative damage in colonic mucosa. TBARS were assessed as marker for lipid peroxidation. However, no differences were observed in colonic mucosal TBARS between the dietary groups. Moreover, TBARS levels seemed similar in non-transgenic and HLA-B27 rats (table 2). Additionally, protein carbonyls were determined in colonic mucosa to evaluate oxidative damage to proteins. Similar to TBARS, diet did not influence the protein carbonyl content of colonic mucosa of transgenic rats. Moreover, protein carbonyls were even lower in transgenic rats compared to non-transgenic rats (table $2 ; \mathrm{p}<0.005$ ).
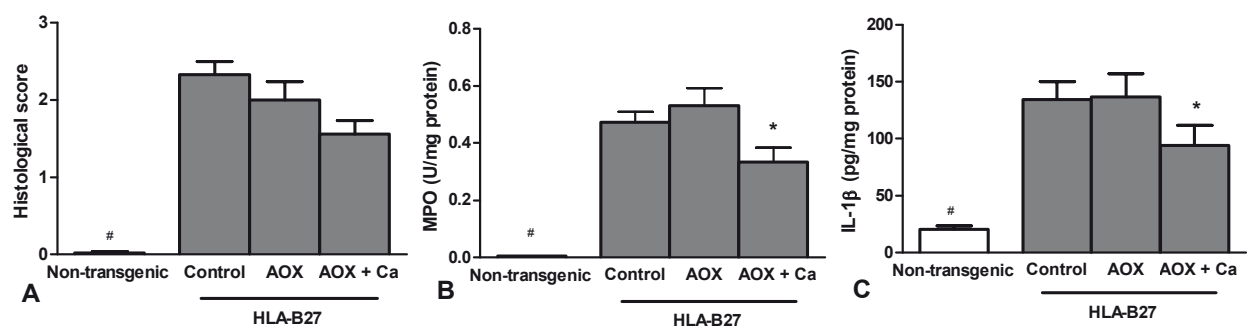

Figure 3: Effect of dietary antioxidants (AOX) and calcium (Ca) on inflammatory parameters in HLA-B27 transgenic rats. Rats were fed the different diets for 9 weeks, after which they were sacrificed to isolate the colonic mucosa for subsequent analyses. (A) Effect on histological inflammation score (range 0-4). (B) Effect

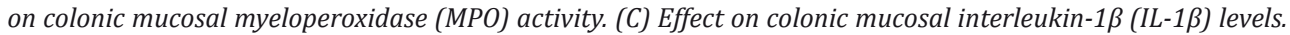
Results are expressed as mean \pm SEM. ${ }^{*}$ Indicates that values in HLA-B27 rats supplemented with both antioxidants and calcium were significantly different from those of HLA-B27 rats fed antioxidants only. "Indicates that nontransgenic rats $(n=7)$ were significantly different from control HLA-B27 rats $(n=9)$. 


\section{Effects on mucosal antioxidant capacity}

To determine whether the dietary antioxidant mixture indeed increased the antioxidant capacity of colonic mucosa, we applied the FRAP assay. Results showed that nonthiol antioxidants were not affected by the different diets. In addition, no substantial differences were observed in the FRAP assay when comparing non-transgenic rats to HLA-B27 rats (table 2). Colonic mucosal glutathione concentrations were measured to determine whether oral glutathione supplementation increases mucosal antioxidant capacity by raising intestinal glutathione levels. Remarkably, glutathione levels were extremely low in colonic mucosa of HLA-B27 rats compared to non-transgenic rats (table 2; $\mathrm{p}<0.0001$ ) and they were not affected by dietary treatment. As we were surprised about the huge difference in glutathione levels, we performed a post-hoc analysis in colonic mucosal samples from our previous study with HLA-B27 rats $^{(14)}$ and found a similar difference between non-transgenic rats $(18.29 \pm 1.15 \mu \mathrm{mol} / \mathrm{g}$ protein) and transgenic rats $(1.74 \pm 0.41 \mu \mathrm{mol} / \mathrm{g}$ protein). To further investigate these exceptionally low mucosal glutathione levels, mucosal cysteine levels were analyzed as well, because cysteine is often the rate-limiting precursor for glutathione synthesis ${ }^{(33)}$. The cysteine concentration in colonic mucosa of transgenic rats was notably high compared to non-transgenic rats and again no dietary effects were observed (table $2 ; \mathrm{p}<0.01$ ). Apparently, more than sufficient cysteine was present in colonic mucosa for the synthesis of glutathione. This may indicate that glutathione synthesis itself is compromised in some way in HLA-B27 transgenic rats. Therefore, we quantified mRNA expression of genes involved in glutathione synthesis. Gene expression of these enzymes (corrected for expression levels of reference genes) in transgenic rats fed the control diet relative to those of their non-transgenic counterparts was 0.8 for $\gamma$-glutamylcysteine synthetase $(\gamma$-GCS) catalytic subunit, 1.7 for $\gamma$-GCS modifier subunit

Table 2: Effect of diet (control, supplemented with an antioxidant mixture (AOX) or supplemented with both antioxidants and calcium $(A O X+C a)$ ) on oxidative stress markers in colonic mucosa of HLA-B27 transgenic rats

\begin{tabular}{lcccc}
\hline & Non-transgenic & \multicolumn{3}{c}{ HLA-B27 } \\
\cline { 3 - 5 } & Control & Control & AOX & AOX + Ca \\
\hline TBARS $(\mu \mathrm{mol} \mathrm{MDA} / \mathrm{mg}$ protein) & $0.70 \pm 0.009$ & $0.72 \pm 0.04$ & $0.72 \pm 0.09$ & $0.67 \pm 0.07$ \\
Carbonyls $(\mathrm{nmol} / \mathrm{mg}$ protein) & $0.67 \pm 0.08^{\#}$ & $0.40 \pm 0.04$ & $0.42 \pm 0.05$ & $0.43 \pm 0.07$ \\
FRAP $\left(\mathrm{nmol} \mathrm{Fe}{ }^{2+} / \mathrm{mg}\right.$ protein) & $30.1 \pm 6.7$ & $40.9 \pm 5.9$ & $48.8 \pm 8.6$ & $37.3 \pm 7.9$ \\
Total GSH $(\mu \mathrm{mol} / \mathrm{g}$ protein) & $13.81 \pm 1.84^{\#}$ & $0.02 \pm 0.002$ & $0.15 \pm 0.13$ & $0.59 \pm 0.38$ \\
Cysteine $(\mu \mathrm{mol} / \mathrm{g}$ protein) & $0.76 \pm 0.51^{\#}$ & $3.60 \pm 1.40$ & $6.53 \pm 1.30$ & $3.52 \pm 1.26$ \\
\hline
\end{tabular}

Rats were fed the different diets for 9 weeks, after which they were sacrificed to isolate the colonic mucosa for subsequent analyses. TBARS are a marker for lipid peroxidation, whereas carbonyls were determined as a marker of oxidative protein damage. Total non-thiol antioxidant capacity was measured by the FRAP assay. Results are expressed as mean \pm SEM. FRAP, ferric reducing ability assay; GSH, glutathione; MDA, malondialdehyde; TBARS, thiobarbiturate reactive substances. ${ }^{\# I n d i c a t e s ~ t h a t ~ n o n-t r a n s g e n i c ~ r a t s ~}(n=7)$ are significantly different from control HLA-B27 rats ( $n=9)$. No significant dietary effects were observed in the HLA-B27 rats. 
$(\mathrm{p}=0.046)$ and 1.3 for glutathione synthetase (GSS). Another potential explanation for the low mucosal glutathione levels is a defect in cellular cysteine uptake resulting in impaired intracellular glutathione synthesis. Interestingly, the cystine transporter in system xc- (xCT), responsible for transporting cystine into the cell in exchange for glutamate, was 6.2-fold upregulated in HLA-B27 rats compared to non-transgenic rats $(\mathrm{p}<0.0001)$.

\section{MP0, glutathione and cysteine levels in young and older HLA-B27 rats}

As the glutathione content of the colonic mucosa of the transgenic rats was remarkably low in comparison to the non-transgenic rats, we were interested to see whether this phenomenon was already present before colitis developed and thus could be a causal factor in this IBD model. Therefore, an additional experiment was performed in which young and older HLA-B27 rats were compared. MPO was measured to quantify colonic mucosal inflammation. At the start of the experiment, MPO levels were slightly higher in transgenic rats $(0.27 \pm 0.11 \mathrm{U} / \mathrm{mg}$ protein $)$ compared to non-transgenic rats $(0.07$ $\pm 0.01 \mathrm{U} / \mathrm{mg}$ protein; $\mathrm{p}<0.005)$. After 10 weeks, levels were further increased in the older HLA-B27 rats $(1.1 \pm 0.14 \mathrm{U} / \mathrm{mg}$ protein; $\mathrm{p}<0.005)$. Interestingly, the colonic mucosal glutathione levels in young HLA-B27 rats were similar to the levels measured in non-transgenic rats (figure 4). However, after 10 weeks, glutathione concentrations decreased in colonic mucosa of transgenic rats compared to those observed in HLA-B27 rats at the start of the experiment (figure $4 ; \mathrm{p}<0.0001$ ). Although the decrease in glutathione was still quite substantial, it was not yet as large as observed in the nutritional intervention study (see table 2). Mucosal cysteine levels were slightly higher in transgenic rats compared to non-transgenic rats at $\mathrm{t}=0$ (figure $4 ; \mathrm{p}<0.001$ ) and were further increased with time in older HLA-B27 rats compared to young HLA-B27 rats (figure 4; $<<0.0001$ ). Since glutathione is predominantly present in the liver ${ }^{(34)}$, we also studied its levels in this organ. The glutathione concentration was similar in the liver of
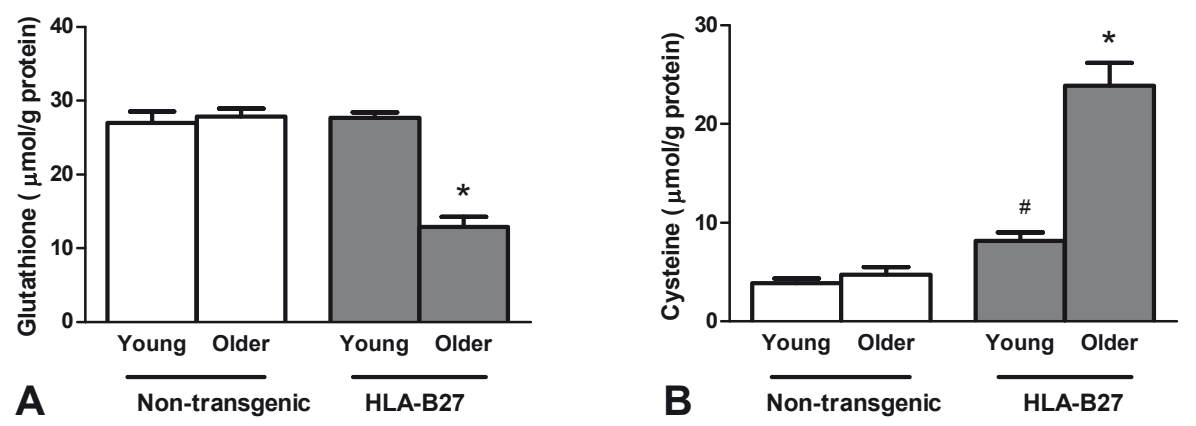

Figure 4: (A) Glutathione and (B) cysteine levels in colonic mucosa of HLA-B27 transgenic rats and their nontransgenic counterparts, determined at a young $(t=0)$ and older $(t=10)$ age. *Indicates that HLA-B27 rats at $t=10(n=9)$ are significantly different from $H L A-B 27$ rats at $t=0 \quad(n=8)$. "Indicates that $H L A-B 27$ rats at $t=0$ are significantly different from non-transgenic rats at $t=0$. 
young transgenic rats $(29.6 \pm 2.7 \mu \mathrm{mol} / \mathrm{g}$ protein $)$ and young non-transgenic rats $(29.0$ $\pm 2.1 \mu \mathrm{mol} / \mathrm{g}$ protein). Comparable levels were observed in non-transgenic rats at $\mathrm{t}=10$ $(29.1 \pm 2.4 \mu \mathrm{mol} / \mathrm{g}$ protein). After 10 weeks, liver glutathione levels were decreased in the transgenic rats $(24.3 \pm 1.7 \mu \mathrm{mol} / \mathrm{g}$ protein), but this was not significant. In summary, the low glutathione levels in colonic mucosa of HLA-B27 rats are only present when intestinal inflammation has developed, and not when the rats were young and still relatively healthy.

\section{ROS production in blood and colonic mucosa}

Another important finding in the nutritional intervention study was the absence of oxidative stress-related damage in the HLA-B27 rats having colitis, despite the low colonic mucosal glutathione levels (see table 2). To examine whether this was due to a defect in ROS production capacity, we measured these ROS in both blood and colonic mucosa in the additional experiment. The ROS production in whole blood was similar in the young non-transgenic and young transgenic rats (table 3). However, in the transgenic rats at $\mathrm{t}=10$ the ability to produce ROS was drastically lower than in nontransgenic rats at $\mathrm{t}=10$ (table $3 ; \mathrm{p}<0.0001$ ). This indicates that the respiratory burst of neutrophils becomes compromised in older HLA-B27 rats. As expected, the amount of neutrophils increased in the older transgenic rats $\left(5.7 \pm 0.8 * 10^{9}\right.$ neutrophils/L; $\mathrm{p}<0.0001)$ compared to the young transgenic rats $\left(1.3 \pm 0.3 * 10^{9}\right.$ neutrophils $\left./ \mathrm{L}\right)$, which reflects the development of colitis. Notwithstanding that, the production of ROS in colonic mucosa did not increase in older HLA-B27 rats compared to young transgenic rats, and was also not different from non-transgenic rats (table 3 ).

\section{DISCUSSION}

In this study we showed that supplementation of an antioxidant mixture had no protective effect on colitis development in HLA-B27 transgenic rats despite very low glutathione levels in colonic mucosa. In addition, we clearly demonstrated that a

Table 3: Reactive oxygen species (ROS) production in blood and colonic mucosa of young and older HLA-B27 transgenic rats compared to non-transgenic rats

\begin{tabular}{llcc}
\hline ROS production & & Non-transgenic & HLA-B27 \\
\hline Blood (RLU/neutrophil) & Young & $105 \pm 17$ & $86 \pm 27$ \\
& Older & $134 \pm 14$ & $27 \pm 3^{*}$ \\
Colonic mucosa $\left({ }^{*} 10^{6} \mathrm{RLU} /\right.$ mg protein) & Young & $1.1 \pm 0.2$ & $1.9 \pm 0.5$ \\
& Older & $1.5 \pm 0.3$ & $2.2 \pm 0.6$ \\
\hline
\end{tabular}

ROS production was quantified by luminol-enhanced chemiluminescence in blood and colonic mucosa of HLA-B27 transgenic rats and their non-transgenic counterparts, both at a young $(t=0)$ and older $(t=10)$ age. Results are expressed as mean $\pm S E M$. RLU, relative light unit. *Indicates that older transgenic rats are significantly different from older non-transgenic rats. 
dietary intervention with both antioxidants and calcium resulted in less severe colitis in HLA-B27 transgenic rats compared to supplementation with the antioxidant mixture only. Supplementation with antioxidants plus calcium significantly attenuated the colitis-related increase in intestinal permeability, diarrhea and inflammatory markers. Overall, these results indicate that only calcium contributed to the protective effects. Furthermore, we showed that colonic glutathione levels decreased when colonic inflammation progresses, suggesting that glutathione deficiency is not a primary cause of colitis development in this rat model. The present study also suggests that the absence of oxidative stress-related mucosal damage in the HLA-B27 rats resulted from compromised ROS production, which might explain the ineffectiveness of antioxidant supplementation.

During active inflammation in IBD, leukocytes massively infiltrate the intestinal mucosa, releasing large amounts of ROS. ROS are thought to be partly responsible for the tissue damage in IBD. To counteract the possible harmful effects of ROS, the intestinal mucosa is equipped with an antioxidant defense system ${ }^{(3)}$. As the balance between the amount of ROS produced and the available antioxidative defense is suggested to be impaired in IBD $^{(35)}$, we investigated the hypothesized relief of inflammatory symptoms in the HLA-B27 rats due to antioxidant supplementation. This study showed that the applied antioxidant mixture neither offered protection to any of the determined characteristics of intestinal inflammation nor affected oxidative stress-related outcomes. One possible explanation for the absence of an antioxidant effect in the present study might be that the transgenic animals did not suffer from mucosal oxidative tissue damage. Indeed, we could not detect signs of oxidative damage in the HLA-B27 rats at the time of tissue collection despite the presence of significant inflammation. Frequently used biomarkers of cellular lipid peroxidation (TBARS) and protein oxidation (carbonyls) were not increased at all in HLA-B27 rats with evident colitis. This shows that intestinal inflammation does not necessarily coincide with evident oxidative stress as is often presumed. To gain further insight into the observed absence of oxidative damage in HLA-B27 rats, we performed a second animal experiment to investigate whether the leukocyte influx indeed caused elevated ROS levels in colonic mucosa. Results showed that there was no increase of ROS production in the colon of transgenic rats suffering from intestinal inflammation. Moreover, in blood the capacity to release ROS was even lower per neutrophilic granulocyte in HLA-B27 rats. So, the absence of increased ROS production in colonic mucosa might explain the lack of oxidative stress-related damage in HLA-B27 rats. Apparently, the observed histological tissue damage in this model is mainly caused by other processes, for instance by activation of matrix metalloproteinases ${ }^{(14,36)}$. Thus the compromised respiratory burst might explain why antioxidant supplementation is not effective in this model.

One of the remarkable findings of the present study is the extremely low colonic mucosal glutathione level in the HLA-B27 transgenic rats. Even this very low glutathione concentration did not lead to measurable oxidative damage. This raises doubts about the importance of colonic mucosal glutathione in preventing oxidative stress. Our finding corroborates a study with rats having intestinal glutathione totally depleted with buthionine sulfoximine, in which also no signs of oxidative damage 
could be detected after intestinal infection ${ }^{(37)}$. Importantly, the absence of an effect of glutathione on oxidative stress in the present study might also result from impaired ROS production. The low intestinal mucosal glutathione levels did not positively respond to oral administration of this thiol tripeptide. To further investigate the glutathione deficit, colonic mucosal mRNA expression of the glutathione synthesis enzymes GSS and $\gamma$-GCS was determined. Levels were similar in transgenic and non-transgenic rats, except for $\gamma$-GCS modifier subunit, which was slightly increased in transgenic rats. Also, availability of precursor amino acids seems sufficient, since cysteine is the ratelimiting substance for glutathione synthesis ${ }^{(38)}$ and colonic mucosal cysteine levels in HLA-B27 rats were even significantly higher compared to non-transgenic rats. Hence, cystine supplementation might have been ineffective to increase mucosal glutathione levels. Furthermore, xCT mRNA expression was notably increased in the transgenic rats. This suggests an increased demand for intracellular cysteine to be used for cellular glutathione synthesis. To follow up on the low glutathione levels, an additional study was performed in which young and older HLA-B27 rats were compared to determine the potential causality of low glutathione to colitis development. Since glutathione levels were not decreased in the young transgenic rats, the intestinal glutathione deficit developed with progression of colitis in these rats. Moreover, the deficit was only present in the colon as liver levels were not significantly decreased. Interestingly, this corresponds with the lower expression of HLA-B27 mRNA in liver compared to colon tissue $^{(16)}$. The cause of the decrease of glutathione in the colonic mucosa is unknown. A defect in the production of NADPH, which is necessary for the reduction of oxidized glutathione, might play role ${ }^{(33)}$. This defect could as well account for the compromised ROS production, since an NADPH-dependent oxidase catalyzes the ROS production by neutrophils ${ }^{(3)}$, but this requires further investigation. Another explanation for the low glutathione levels in the transgenic rats might be the presence of inhibitors of glutathione synthesis, like cysteamine ${ }^{(39-40)}$. In the nutritional intervention study and our previous HLA-B27 study(14), elevated cysteamine levels in colonic mucosa were observed (data not shown). However, in the additional experiment with young and older rats, no cysteamine was detected in the colonic mucosa, indicating that its role, if any, is inconsistent. Possibly, other inhibitors are implicated in the low glutathione levels of the transgenic rats ${ }^{(40)}$. Remarkably, El Yousfi et al report increased glutathione levels in the colon of HLA-B27 rats compared to non-transgenic rats ${ }^{(41)}$ but the expression units and colonic part (mucosa or whole colon) are unclear. Therefore, it is difficult to compare their results with ours, which were consistent in three independent HLA-B27 studies.

For better understanding of the HLA-B27 model and its application to study mechanisms relevant to human IBD, it is important to investigate whether the compromised capacity of neutrophils to produce ROS together with the low colonic glutathione levels plays a role in the development of colitis in this model. As HLA-B27-expressing innate immune cells have an essential role in this model ${ }^{(17)}$, we speculate that these cells do not adequately respond to invading bacteria due to their compromised ROS production, which might lead to an overload of antigen presentation to T cells, resulting in an overreactive immune system. Further research is necessary to support this hypothesis. 
The beneficial effect of dietary calcium on colitis development in HLA-B27 transgenic rats was shown before by our group ${ }^{(14)}$. Additionally, we have shown in several controlled rat and human infection studies that dietary calcium improves intestinal resistance and strengthens the mucosal barrier ${ }^{(18-20)}$. Since studies in the field of colon cancer research have shown that calcium affects intestinal epithelial cell homeostasis ${ }^{(42-43)}$, cell cycle function was also analyzed in mucosal samples from the present study. The dietary treatments did not affect either cell proliferation (Ki-67 immunohistochemistry) or apoptosis (caspase-3 activity) (data not shown). These results suggest that the protective influence of calcium on colitis symptoms, e.g. intestinal permeability, diarrhea and inflammation markers, cannot be explained by effects on mucosal cell proliferation and apoptosis. We hypothesize that the beneficial effect of calcium involves precipitation of cytotoxic surfactants by luminal calcium and thus modulation of the intestinal microbiota.

In conclusion, supplementation of a mixture of various antioxidants had no effect on colitis severity in HLA-B27 transgenic rats, whereas the significantly protective effect of dietary calcium shown before was corroborated by this study. Very low glutathione levels were observed in colonic mucosa of HLA-B27 rats, which developed during progression of intestinal inflammation. In addition, compromised ROS production might explain why there is no oxidative stress-related mucosal damage in the transgenic rats and why antioxidant supplementation was ineffective. Based on the consistent beneficial intestinal effects of calcium, a clinical trial with IBD patients to demonstrate proof-ofconcept in humans might be interesting. The presumed absence of adverse effects of nutritional interventions, in contrast to commonly prescribed IBD medication, makes it worthwhile to further explore the potential benefits of nutritional intervention in intestinal inflammation in humans.

\section{Acknowledgements}

The authors wish to thank the biotechnicians of the Small Animal Centre (Wageningen University and Research Centre, The Netherlands) for expert assistance.

\section{REFERENCES}

1. Sartor RB. Mechanisms of disease: pathogenesis of Crohn's disease and ulcerative colitis. Nat Clin Pract Gastroenterol Hepatol. 2006;3:390-407.

2. Ling SC, Griffiths AM. Nutrition in inflammatory bowel disease. Curr Opin Clin Nutr Metab Care. 2000;3:339-344.

3. Kruidenier L, Verspaget HW. Review article: oxidative stress as a pathogenic factor in inflammatory bowel disease--radicals or ridiculous? Aliment Pharmacol Ther. 2002;16:1997-2015.

4. Geerling BJ, Stockbrugger RW, Brummer RJ. Nutrition and inflammatory bowel disease: an update. Scand J Gastroenterol Suppl. 1999;230:95-105.

5. Blomhoff R, Carlsen MH, Andersen LF, et al. Health benefits of nuts: potential role of antioxidants. $\mathrm{Br} \mathrm{J}$ Nutr. 2006;96 Suppl 2:S52-60.

6. $\quad$ Fang YZ, Yang S, Wu G. Free radicals, antioxidants, and nutrition. Nutrition. 2002;18:872-879.

7. Packer L, Weber SU, Rimbach G. Molecular aspects of alpha-tocotrienol antioxidant action and cell signalling. J Nutr. 2001;131:369S-373S.

8. Martensson J, Meister A. Glutathione deficiency decreases tissue ascorbate levels in newborn rats: 
ascorbate spares glutathione and protects. Proc Natl Acad Sci U S A. 1991;88:4656-4660.

9. Martensson J, Meister A. Glutathione deficiency increases hepatic ascorbic acid synthesis in adult mice. Proc Natl Acad Sci U S A. 1992;89:11566-11568.

10. Meister A. Glutathione-ascorbic acid antioxidant system in animals. J Biol Chem. 1994;269:9397-9400.

11. Favilli F, Marraccini P, Iantomasi T, et al. Effect of orally administered glutathione on glutathione levels in some organs of rats: role of specific transporters. Br J Nutr. 1997;78:293-300.

12. Martensson J, Jain A, Meister A. Glutathione is required for intestinal function. Proc Natl Acad Sci U S A. 1990;87:1715-1719.

13. Valencia E, Hardy G. Practicalities of glutathione supplementation in nutritional support. Curr Opin Clin Nutr Metab Care. 2002;5:321-326.

14. Schepens MA, Schonewille AJ, Vink C, et al. Supplemental calcium attenuates the colitis-related increase in diarrhea, intestinal permeability, and extracellular matrix breakdown in HLA-B27 transgenic rats. J Nutr. 2009;139:1525-1533.

15. Sartor RB. Colitis in HLA-B27/beta 2 microglobulin transgenic rats. Int Rev Immunol. 2000;19:39-50.

16. Hammer RE, Maika SD, Richardson JA, et al. Spontaneous inflammatory disease in transgenic rats expressing HLA-B27 and human beta 2m: an animal model of HLA-B27-associated human disorders. Cell. 1990;63:1099-1112.

17. Hoentjen F, Tonkonogy SL, Liu B, et al. Adoptive transfer of nontransgenic mesenteric lymph node cells induces colitis in athymic HLA-B27 transgenic nude rats. Clin Exp Immunol. 2006;143:474-483.

18. Bovee-Oudenhoven IM, Lettink-Wissink ML, Van Doesburg W, et al. Diarrhea caused by enterotoxigenic Escherichia coli infection of humans is inhibited by dietary calcium. Gastroenterology. 2003;125:469476.

19. Bovee-Oudenhoven IM, Termont DS, Weerkamp AH, et al. Dietary calcium inhibits the intestinal colonization and translocation of Salmonella in rats. Gastroenterology. 1997;113:550-557.

20. Ten Bruggencate SJ, Bovee-Oudenhoven IM, Lettink-Wissink ML, et al. Dietary fructo-oligosaccharides and inulin decrease resistance of rats to salmonella: protective role of calcium. Gut. 2004;53:530-535.

21. Govers MJ, Termont DS, Lapre JA, et al. Calcium in milk products precipitates intestinal fatty acids and secondary bile acids and thus inhibits colonic cytotoxicity in humans. Cancer Res. 1996;56:3270-3275.

22. Lapre JA, De Vries HT, Koeman JH, et al. The antiproliferative effect of dietary calcium on colonic epithelium is mediated by luminal surfactants and dependent on the type of dietary fat. Cancer Res. 1993;53:784-789.

23. Reeves PG, Nielsen FH, Fahey GC, Jr. AIN-93 purified diets for laboratory rodents: final report of the American Institute of Nutrition ad hoc writing committee on the reformulation of the AIN-76A rodent diet. J Nutr. 1993;123:1939-1951.

24. Arslan G, Atasever T, Cindoruk M, et al. (51)CrEDTA colonic permeability and therapy response in patients with ulcerative colitis. Nucl Med Commun. 2001;22:997-1001.

25. Binnerts W, Van het Klooster A, Frens A. Soluble chromium indicator measured by atomic absorption in digestion experiments. Vet Rec. 1968;82:470.

26. Rath HC, Herfarth HH, Ikeda JS, et al. Normal luminal bacteria, especially Bacteroides species, mediate chronic colitis, gastritis, and arthritis in HLA-B27/human beta2 microglobulin transgenic rats. J Clin Invest. 1996;98:945-953.

27. Grisham MB, Benoit JN, Granger DN. Assessment of leukocyte involvement during ischemia and reperfusion of intestine. Methods Enzymol. 1990;186:729-742.

28. Ohkawa H, Ohishi N, Yagi K. Assay for lipid peroxides in animal tissues by thiobarbituric acid reaction. Anal Biochem. 1979;95:351-358.

29. Buss H, Chan TP, Sluis KB, et al. Protein carbonyl measurement by a sensitive ELISA method. Free Radic Biol Med. 1997;23:361-366.

30. Benzie IF, Strain JJ. The ferric reducing ability of plasma (FRAP) as a measure of "antioxidant power": the FRAP assay. Anal Biochem. 1996;239:70-76.

31. Mansoor MA, Svardal AM, Ueland PM. Determination of the in vivo redox status of cysteine, cysteinylglycine, homocysteine, and glutathione in human plasma. Anal Biochem. 1992;200:218-229.

32. van der Logt EM, Roelofs HM, Wobbes T, et al. High oxygen radical production in patients with sporadic colorectal cancer. Free Radic Biol Med. 2005;39:182-187.

33. Meister A. Glutathione deficiency produced by inhibition of its synthesis, and its reversal; applications 
in research and therapy. Pharmacol Ther. 1991;51:155-194.

34. Kosower NS, Kosower EM. The glutathione status of cells. Int Rev Cytol. 1978;54:109-160.

35. Kruidenier L, Verspaget HW. Antioxidants and mucosa protectives: realistic therapeutic options in inflammatory bowel disease? Mediators Inflamm. 1998;7:157-162.

36. Medina C, Radomski MW. Role of matrix metalloproteinases in intestinal inflammation. J Pharmacol Exp Ther. 2006;318:933-938.

37. van Ampting MT, Schonewille AJ, Vink C, et al. Intestinal barrier function in response to abundant or depleted mucosal glutathione in Salmonella-infected rats. BMC Physiol. 2009;9:6.

38. Griffith OW. Biologic and pharmacologic regulation of mammalian glutathione synthesis. Free Radic Biol Med. 1999;27:922-935.

39. Berruyer C, Pouyet L, Millet V, et al. Vanin-1 licenses inflammatory mediator production by gut epithelial cells and controls colitis by antagonizing peroxisome proliferator-activated receptor gamma activity. J Exp Med. 2006;203:2817-2827.

40. Griffith OW, Mulcahy RT. The enzymes of glutathione synthesis: gamma-glutamylcysteine synthetase. Adv Enzymol Relat Areas Mol Biol. 1999;73:209-267, xii.

41. El Yousfi M, Breuille D, Papet I, et al. Increased tissue protein synthesis during spontaneous inflammatory bowel disease in HLA-B27 rats. Clin Sci (Lond). 2003;105:437-446.

42. Lipkin M, Newmark H. Calcium and the prevention of colon cancer.J Cell Biochem Suppl. 1995;22:65-73.

43. Pence BC. Role of calcium in colon cancer prevention: experimental and clinical studies. Mutat Res. 1993;290:87-95. 


\title{
Dietary calcium decreases but short-chain fructo-oligosaccharides increase colonic permeability in rats
}

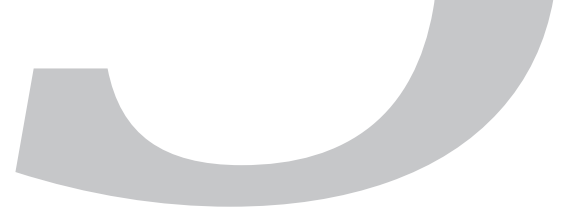

\author{
Marloes A. A. Schepens \\ Anneke Rijnierse \\ Arjan J. Schonewille \\ Carolien Vink \\ Robert-Jan M. Brummer \\ Linette E. M. Willemsen \\ Roelof van der Meer \\ Ingeborg M. J. Bovee-Oudenhoven
}




\section{Abstract}

Objective: An increased intestinal permeability is associated with several diseases. Nutrition can influence gut permeability. Previously, we showed that dietary Ca decreases whereas dietary short-chain fructo-oligosaccharides (scFOS) increase intestinal permeability in rats. However, it is unknown how and where in the gastrointestinal tract Ca and scFOS exert their effects.

Methods: Rats were fed a Western low-Ca control diet, or a similar diet supplemented with either Ca or scFOS. Lactulose plus mannitol and Cr-EDTA were added to the diets to quantify small and total gastrointestinal permeability, respectively. Additionally, colonic tissue was mounted in Ussing chambers and exposed to faecal water of these rats.

Results: Dietary Ca immediately decreased urinary Cr-EDTA excretion by $24 \%$ in Ca-fed rats compared with control rats. Dietary scFOS increased total Cr-EDTA permeability gradually with time, likely reflecting relatively slow gut microbiota adaptations, which finally resulted in a 30\% increase. The lactulose:mannitol ratio was $15 \%$ higher for Cafed rats and $16 \%$ lower for scFOS-fed rats compared with control rats. However, no dietary effect was present on individual urinary lactulose and mannitol excretion. The faecal waters did not influence colonic permeability in Ussing chambers.

Conclusion: In conclusion, despite effects on the lactulose:mannitol ratio, individual lactulose values did not alter, indicating that diet did not influence small-intestinal permeability. Therefore, both nutrients affect permeability only in the colon: Ca decreases, while scFOS increase colonic permeability. As faecal water did not influence permeability in Ussing chambers, probably modulation of mucins and/or microbiota is important for the in vivo effects of dietary Ca and scFOS. 


\section{INTRODUCTION}

The intestinal epithelial cell layer constitutes the largest and most important barrier against the external environment. Tight contact between the enterocytes prevents access of intraluminal toxins, antigens and enteric microbiota to underlying tissue compartments. A compromised intestinal barrier function is suggested to be associated with the pathogenesis of a number of intestinal diseases including inflammatory bowel disease, coeliac disease, post-infectious irritable bowel syndrome and food allergy ${ }^{(1-4)}$. Accumulating evidence about the progression of inflammatory bowel disease, a chronic relapsing and remitting disease for which the aetiology remains unknown, shows that an increased intestinal permeability intensifies the exposure of the lamina propria to luminal contents, activating an abnormal immune response $e^{(3,5)}$.

Interestingly, nutrition can affect the epithelial barrier, and hence possibly influence disease development. We have shown in several controlled studies that dietary Ca protects against intestinal infections with foodborne pathogens ${ }^{(6-8)}$. Ca also has cytoprotective effects by precipitating cytotoxic surfactants, such as secondary bile acids $^{(9,10)}$. In addition, supplemental $\mathrm{Ca}$ attenuates the development of colitis $^{(11)}$. In the latter study, total intestinal permeability was quantified by the analysis of urinary Cr-EDTA excretion. We observed that supplemental Ca prevented the colitis-related increase in intestinal permeability in HLA-B27 transgenic rats ${ }^{(11)}$. Another example of dietary modulation of intestinal permeability is the supplementation of short-chain fructooligosaccharides (scFOS), which may be regarded as a prebiotic. Work from our group has shown that these dietary scFOS impair the resistance to intestinal infection in $\operatorname{rats}^{(8,12,13)}$. Subsequently, we showed that this decrease in barrier functioning was associated with a higher total intestinal permeability in rats ${ }^{(14)}$.

The dietary effects on intestinal permeability in the studies mentioned earlier were investigated by the addition of Cr-EDTA to the diet and subsequent measurement of urinary Cr-EDTA excretion. As Cr-EDTA is stable throughout the intestinal tract, the urinary excretion of this marker provides information regarding total intestinal permeability ${ }^{(15)}$. By choosing permeability probes that have limited exposure to certain parts of the intestinal epithelium, region-specific permeability measurements can be performed ${ }^{(16)}$. For example, the saccharide probes lactulose and mannitol can be used to measure small-intestinal permeability, because they are instantly degraded by the colonic microbiota. Since lactulose only passes the gut wall paracellularly and mannitol passes transcellularly, the lactulose:mannitol ratio is used to express small-intestinal permeability ${ }^{(2)}$.

We hypothesised that both a high-Ca diet and a scFOS diet mainly influence colonic permeability. As colitis is suggested to result from increased translocation of microorganisms due to a higher colonic permeability and Ca attenuated colitis development ${ }^{(11)}$, dietary Ca probably has its main effect on permeability of the colon. Since scFOS are fermented in the colon, where they change the luminal content, which possibly influences the colonic mucosa, we also hypothesised that scFOS mainly affect colonic permeability. The aim of the present study was to localise these effects. Therefore, we 
added Cr-EDTA, lactulose and mannitol as permeability markers to the rat diets. As no specific colonic permeability marker exists, this can be determined by comparing total (Cr-EDTA) and small (lactulose:mannitol ratio) intestinal permeability. To gain even more insight into the effect of dietary Ca and scFOS on permeability, we performed Ussing chamber experiments with the faecal waters derived from the in vivo study.

\section{MATERIAL AND METHODS}

\section{Experimental design of the nutritional study: animals and diets}

The experimental protocol was approved by the Animal Welfare Committee of Wageningen University and Research Centre (Wageningen, The Netherlands). Specific pathogenfree outbred male Wistar rats (WU, Harlan, Horst, The Netherlands), 8 weeks old and with a mean body weight of $267 \mathrm{~g}$ at the start of the experiment, were housed individually in metabolic cages. Rats were maintained in temperature- and humiditycontrolled environment in a $12 \mathrm{~h}$ light-dark cycle. Rats were fed ad libitum a purified 'humanised' Western diet which contained in the control situation (per kg): $200 \mathrm{~g}$ acid casein, 326 g maize starch, 162 g glucose, 160 g palm oil, 40 g maize oil, 50 g cellulose and $5 \cdot 16 \mathrm{~g} \mathrm{CaHPO}_{4} 2 \mathrm{H}_{2} \mathrm{O}$ (corresponding to $30 \mathrm{mmol} \mathrm{Ca} / \mathrm{kg}$ diet; Sigma-Aldrich, St Louis, MO, USA). In addition, the diets contained the following permeability markers per kg: 2 g Cr-EDTA, 7.5 g lactulose (Solvay Arzneimittel GmbH, Hannover, Germany) and $2.5 \mathrm{~g}$ mannitol (Sigma-Aldrich) (see below). Lactulose and mannitol were added to the diet to avoid stressful supplementation by oral gavage which might have an impact on intestinal permeability and transit time. Moreover, the continuous exposure of the rats to the permeability probes excludes a possible day-night rhythm in intestinal permeability. Vitamins and minerals (other than $\mathrm{Ca}$ ) were added to both the diets according to AIN-93 ${ }^{(17)}$. This control diet was low in Ca and had a high fat content to mimic the composition of a Western human diet. The diet high in Ca contained 120 $\mathrm{mmol} \mathrm{Ca} / \mathrm{kg}$ diet, and in the scFOS group, the control diet was supplemented with $60 \mathrm{~g}$ scFOS/kg diet (6\% (w/w); Raftilose $®$ P95, Orafti, Tienen, Belgium), both at the expense of glucose. The degree of polymerisation of the supplemented scFOS ranges between 2 and 8 , and therefore they are classified as scFOS. Inert Cr-EDTA was added to the diets to quantify total intestinal permeability ${ }^{(18)}$. Cr-EDTA solution was prepared as described elsewhere and subsequently freeze dried ${ }^{(19)}$. To check the complete formation of the $\mathrm{Cr}$ EDTA complex, the prepared Cr-EDTA solution was passed through a cation-exchange resin column (Chelex 100 Resin; Bio-Rad, Hercules, CA, USA). No uncomplexed $\mathrm{Cr}^{3+}$ ions were present. Potential binding and subsequent precipitation of Cr-EDTA to Ca were determined by quantification of the Cr-EDTA concentration in the faecal water. This was similar in the different dietary groups, so binding of Cr-EDTA to Ca was concluded to be absent. Small-intestinal permeability was assessed using lactulose and mannitol ${ }^{(2,20)}$. Food intake was recorded daily, and animal weight was recorded three times every week. After $14 \mathrm{~d}$ of experimental feeding, rats were anaesthetised with isoflurane and killed. 


\section{Measurement of intestinal permeability}

Total $24 \mathrm{~h}$ urine samples were collected every $2 \mathrm{~d}$ during the experiment. For Cr-EDTA measurement, urine was acidified with $50 \mathrm{~g} / \mathrm{l} \mathrm{TCA}$, centrifuged for $2 \mathrm{~min}$ at $14000 \mathrm{~g}$ and diluted with $0.5 \mathrm{~g} / \mathrm{l} \mathrm{CsCl}$. Then, $\mathrm{Cr}$ was analysed by inductively coupled plasma-atomic emission spectrophotometry. Urinary lactulose and mannitol were analysed by highperformance anion-exchange chromatography with pulsed amperometric detection on a Au electrode. The analyses were performed with a 600E System controller pump (Waters, Milford, MA, USA) with a He degassing unit and a model 400 EC detector (EG\&G, Princeton, NJ, USA). With a WPS-3000 autosampler (Dionex, Sunnyvale, CA, USA), $20 \mu \mathrm{l}$ of the (diluted) sample were injected on a Carbopac MA-1, $250 \times 4 \mathrm{~mm}$, column (Dionex) thermostated at $30^{\circ} \mathrm{C}$. Lactulose and mannitol were eluted isocratically at a flow rate of $0.40 \mathrm{ml} / \mathrm{min}$ with $480 \mathrm{mM}-\mathrm{NaOH}$ followed by a washing step of $1000 \mathrm{mM}-\mathrm{NaOH}$ and $1000 \mathrm{mM}$-sodium acetate. Data analysis was done with Chromeleon software version 6.60 (Dionex).

\section{Ussing chambers}

All faeces were collected on the last $3 \mathrm{~d}$ of the nutritional experiment and freeze dried. Faecal water was prepared as described earlier ${ }^{(21)}$. The animal welfare committee of Utrecht University (Utrecht, The Netherlands) approved the animal protocol. Specific pathogen-free outbred male Wistar rats $\left(\mathrm{n}=6\right.$; WU), 7-8 weeks old, were killed by $\mathrm{CO}_{2}$ inhalation. The colon was taken out and washed in Ringer solution $\left(25 \mathrm{mM}-\mathrm{NaHCO}_{3}\right.$, $117 \cdot 5 \mathrm{mM}-\mathrm{NaCl}, 1 \cdot 25 \mathrm{mM}-\mathrm{CaCl}_{2}, 5 \cdot 7 \mathrm{mM}-\mathrm{KCl}, 1 \cdot 2 \mathrm{mM}-\mathrm{NaH}_{2} \mathrm{PO}_{4}, 1 \cdot 2 \mathrm{mM}-\mathrm{MgSO}_{4}$ and 5 g glucose, $\mathrm{pH} 7.4$ when gassed with $95 \% \mathrm{O}_{2}-5 \% \mathrm{CO}_{2}$ at $37^{\circ} \mathrm{C}$ ), and its muscle layers were stripped off. The tissue sections were cut into specimens of appropriate size and randomly mounted in Ussing chambers (manufactured by Technical Services of Utrecht Institute for Pharmaceutical Sciences), each side containing the Ringer solution. After a $30 \mathrm{~min}$ equilibration period, baseline transepithelial resistance was measured, and the Ringer solution in the mucosal compartment was replaced by fresh Ringer solution (negative control), or Ringer solution containing no $\mathrm{Ca}$, or faecal water prepared from faeces of the different dietary groups of the nutritional study described earlier. Colonic tissue was thus exposed to faecal water pools of either control rats, Ca-fed rats or scFOS-fed rats. The Ca chelator ethylene glycol tetraacetic acid served as a positive control for enhanced permeability due to Ca binding ( $8 \mathrm{mM}$ as final concentration in Ringer solution in both compartments). At the same time, fluorescein isothiocyanatelabelled $4 \mathrm{kDa}$ dextran (FITC-dextran) was added at the mucosal side $(1 \mathrm{mg} / \mathrm{ml}$ as final concentration). Samples were taken every $30 \mathrm{~min}$ from the serosal compartment for subsequent fluorescence measurement and replaced by fresh Ringer solution. The experiment was carried out for $180 \mathrm{~min}$, after which endline transepithelial resistance was determined. Flux was expressed as nanomol of FITC-dextran crossing $1 \mathrm{~cm}^{2}$ of colonic epithelium in $1 \mathrm{~h}\left(\mathrm{nmol} / \mathrm{cm}^{2}\right.$ per $\left.\mathrm{h}\right)$.

\section{Statistical analysis}

All the results are expressed as means with their standard errors. The predefined comparisons of interest for the nutritional study were control rats v. Ca-fed rats and 
control rats v. scFOS-fed rats. For the in vitro experiments, the predefined comparisons of interest were: faecal water of control rats v. faecal water of Ca-fed rats, faecal water of control rats $\mathrm{v}$. faecal water of scFOS-fed rats and the negative control v. withdrawal of Ca from the Ringer solution. Statistics were done by one-way ANOVA or KruskalWallis test, depending on the normality of the data. If significant, this was followed by Student's t test (for normally distributed data) or Mann-Whitney U test (for nonnormally distributed data) to identify the significant dietary effects. Differences were considered statistically significant when $\mathrm{P}<0 \cdot 05$ (all two-sided). Statistical analyses were conducted with GraphPad Prism version 5.01 (GraphPad Software, Inc., La Jolla, CA, USA).

\section{RESULTS}

\section{Animal growth and food intake}

Rats fed the high-Ca diet did not differ from rats fed the low-Ca control diet with respect to growth (5.8 (SE 0.3) and 6.3 (SE 0.3) g/d, respectively; $\mathrm{P}=0 \cdot 16)$. However, animal growth in the scFOS group was significantly lower $(4 \cdot 7$ (SE $0 \cdot 3) \mathrm{g} / \mathrm{d}, \mathrm{P}=0 \cdot 0002)$ in accordance with a previous study ${ }^{(12)}$. Food intake of rats fed the low-Ca control diet and of rats fed the high-Ca diet was similar (21.6 (SE 0.4) and 21.4 (SE 0.5) g/d, respectively; $\mathrm{P}=0 \cdot 72$ ), while food intake of the scFOS-fed rats was significantly less than that of control rats $(18 \cdot 4(\mathrm{SE} 0 \cdot 4) \mathrm{g} / \mathrm{d}, \mathrm{P}<0 \cdot 0001)$.

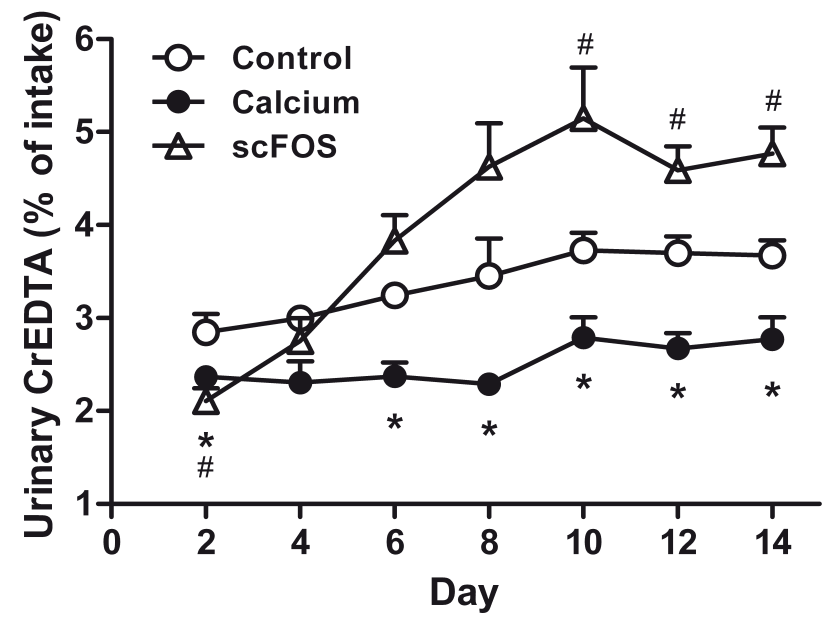

Figure 1: Effect of dietary Ca and short-chain fructo-oligosaccharides (scFOS) on urinary Cr-EDTA excretion, a marker for total intestinal paracellular permeability. Results are expressed as means with their standard errors $(n=13)$. ${ }^{*}$ Mean values of Ca-fed rats were significantly different compared with control rats $(P<0 \cdot 05)$. ${ }^{*}$ Mean values of ScFOS-fed rats were significantly different compared with control rats $(P<0 \cdot 05)$. 


\section{Dietary calcium and short-chain fructo-oligosaccharides influence total intestinal permeability}

Dietary Ca supplementation caused a direct and persistent decrease in urinary Cr-EDTA excretion; the decrease was $24 \%$ in rats fed the high-Ca diet compared with rats fed the low-Ca control diet on day $14(\mathrm{P}<0.05$; Figure 1$)$. Urinary excretion of Cr-EDTA by scFOS-fed rats was lower than that by control rats at the beginning of the experiment (day 2). This might represent shorter exposure of the intestinal epithelium to luminal contents due to more rapid gut transit on the scFOS diet, which has been observed in past studies ${ }^{(22)}$. From day 2, urinary Cr-EDTA excretion by scFOS-fed rats increased gradually with time compared with the control group, probably reflecting relatively slow gut microbiota adaptations due to scFOS. After 2 weeks, dietary scFOS had increased total intestinal permeability by $30 \%(\mathrm{P}<0 \cdot 05$; Figure 1$)$.

\section{Effect of calcium and short-chain fructo-oligosaccharides on small-intestinal permeability}

Dietary effects on the lactulose:mannitol ratio were smaller and, surprisingly, in the opposite direction compared to total intestinal permeability as measured by Cr-EDTA. The lactulose:mannitol ratio on day 14 was $15 \%$ higher for rats fed the high-Ca diet $(0 \cdot 23$ (SE 0.006), $\mathrm{P}=0 \cdot 0014)$ and $16 \%$ lower for rats fed the scFOS diet $(0 \cdot 17$ (SE 0.009), $\mathrm{P}=0 \cdot 0038)$ compared with rats fed the low-Ca control diet $(0 \cdot 20(\mathrm{SE} 0 \cdot 005))$.

We also considered the lactulose and mannitol fluxes individually to gain more insight and specify whether paracellular permeability was changed. However, we did not observe significant dietary effects on individual lactulose $(\mathrm{P}=0 \cdot 61)$ and mannitol

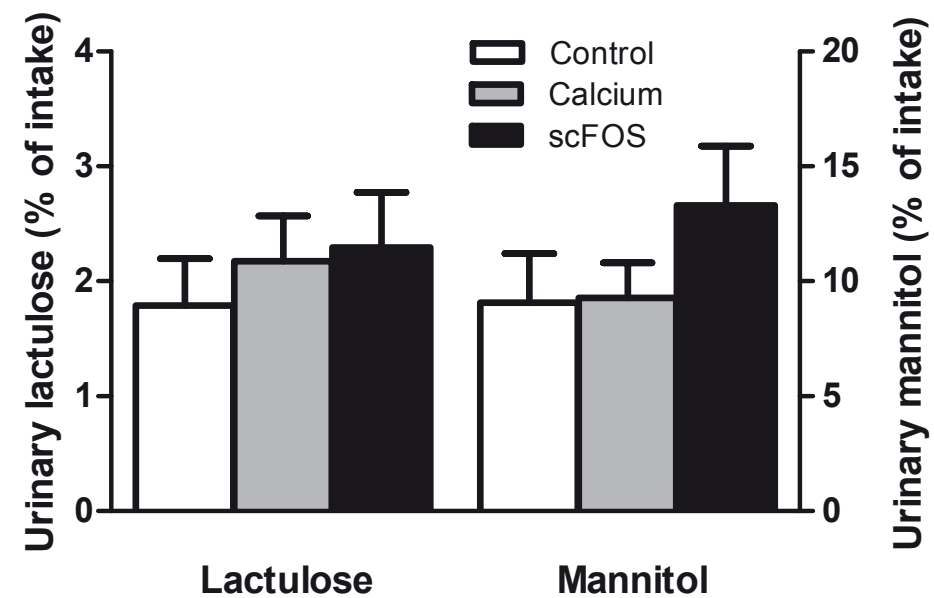

Figure 2: Effect of dietary Ca and short-chain fructo-oligosaccharides (scFOS) on individual urinary lactulose and mannitol excretion. Results are expressed as means with their standard errors $(n=13)$. 
excretion in urine $(\mathrm{P}=0 \cdot 36$; Figure 2$)$. This indicates that paracellular permeability in the small intestine remained the same. In combination with the Cr-EDTA results, it can therefore be concluded that both Ca and scFOS affect permeability only in the colon.

\section{Effect of faecal water from rats fed supplemental calcium or short-chain fructo- oligosaccharides in Ussing chambers}

Faecal waters were prepared from faeces of the in vivo rat experiment and tested in Ussing chambers using colonic tissue. Diet influenced the $\mathrm{pH}$ of pools of faecal water (control: 6.1; Ca: 6.5 and scFOS: 5.5). No dietary effects were observed on transepithelial resistance $(\mathrm{P}=0 \cdot 23)$, while the positive control (ethylene glycol tetraacetic acid) caused a decrease in resistance as expected ( $\mathrm{P}=0.0019$; data not shown). Also, the paracellular flux of FITC-dextran crossing the epithelial barrier was similar for all faecal waters at all time points $(\mathrm{P}=0.67$; Figure 3$)$. Obviously, the FITC-dextran flux was increased in the positive control due to the addition of the Ca chelator ethylene glycol tetraacetic acid $(\mathrm{P}=0.0002)$. So, diet-modified faecal waters did not influence colonic permeability in Ussing chambers. Furthermore, the Ca-free Ringer solution at the luminal side of the Ussing chamber did not influence dextran flux either ( $\mathrm{P}=0.09$; Figure 3$)$.

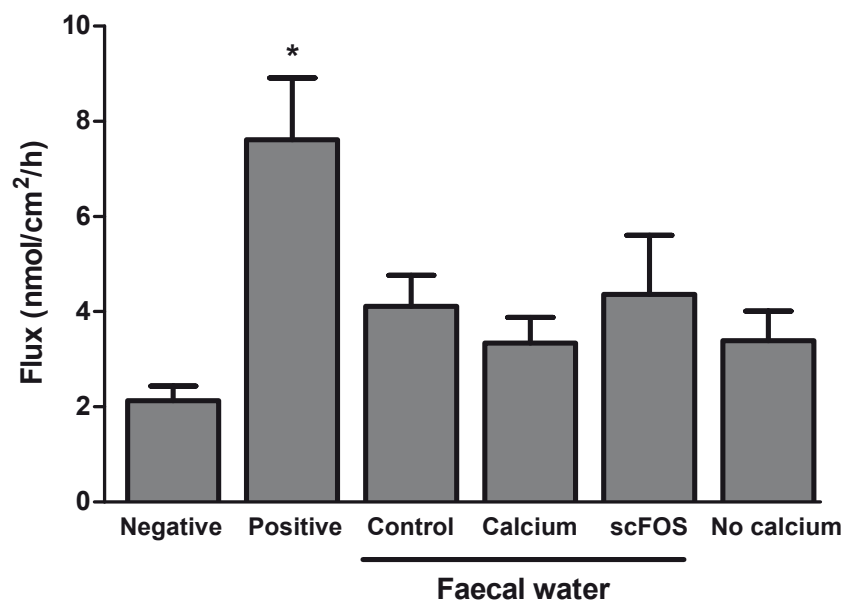

Figure 3: Effect of faecal water from rats fed a control diet, Ca-supplemented diet or short-chain fructooligosaccharides (scFOS)-supplemented diet on fluorescein isothiocyanate-labelled $4 \mathrm{kDa}$ dextran (FITCdextran) flux, a marker for permeability, across stripped colonic tissue in Ussing chambers. Also, the Ca-free Ringer solution at the luminal side of the Ussing chamber was studied ('no Calcium'). Results (means with their standard errors) are expressed as nanomol of FITC-dextran crossing $1 \mathrm{~cm}^{2}$ of colonic epithelium in $1 \mathrm{~h}$. The interval of time shown (60-90 $\mathrm{min}$ ) is representative for the whole experiment. Negative (Ringer), $n=12$; positive (ethylene glycol tetraacetic acid in Ringer), $n=10$; faecal water of control rats, $n=9$; faecal water of Cafed rats, $n=10$; faecal water of scFOS-fed rats, $n=9$; no Calcium $(n=13)$. *Mean values were significantly different compared with negative control $(P=0 \cdot 0002)$. 


\section{DISCUSSION}

The present study demonstrates that the effect of dietary Ca and scFOS on permeability in rats is localised in the colon. Supplemental Ca clearly decreased total intestinal permeability, as determined by Cr-EDTA as a marker. At the same time, it caused a less pronounced increase in the lactulose:mannitol ratio but without affecting the individual lactulose excretion, representing small-bowel permeability. Taken together, it can be concluded, although indirectly, that the overall permeability is decreased by $\mathrm{Ca}$ supplementation and that the effect is localised in the colon. The same applies for scFOS, although the results are opposite: dietary scFOS increased total intestinal permeability as measured by Cr-EDTA, whereas a minor decrease in the lactulose:mannitol ratio was observed without an effect on individual urinary lactulose values. Therefore, we can indirectly conclude that the increase in permeability caused by scFOS is also localised in the colon. The Ussing chamber experiments showed that dietary Ca and scFOS did not influence colonic permeability via alterations in faecal water.

The consistent finding that dietary Ca beneficially influences colonic permeability is very interesting. In the first place, it is intriguing that intestinal permeability apparently can be improved in a healthy situation. Secondly, an increased permeability is suggested to be a primary causative factor contributing to inflammatory bowel disease pathogenesis ${ }^{(2,23,24)}$. It is generally accepted that the gut microbiota and its intimate contact with an over-reactive immune system in the intestinal epithelium is the driving force behind the chronic inflammation ${ }^{(25)}$. As intestinal bacteria reside mainly in the colon ${ }^{(26)}$, decreasing of colonic permeability by a high-Ca diet might be a highly attractive nutritional intervention for inflammatory bowel disease patients. Moreover, in a previous study, we showed that supplemental Ca attenuated some important aspects of colitis development in HLA-B27 transgenic rats ${ }^{(11)}$.

Based on the individual lactulose results, dietary Ca does not influence small-intestinal permeability. The fact that Meddings \& Gibbons ${ }^{(16)}$ could not detect any lactulose and mannitol in the colon underlines the specificity of both lactulose and mannitol as markers for small-intestinal permeability. Therefore, Ca supplementation might only be worthwhile when targeting the colon for health improvement. The reason for the difference in localisation is unknown, but we hypothesise that Ca influences intestinal permeability by modulating the colonic microbiota or its metabolites. For example, Salmonella typhi and Escherichia coli, being members of the enterobacteria, are able to induce an increase in intestinal permeability ${ }^{(27)}$. In agreement with these results, dietary Ca decreased faecal enterobacteria in the present study (control: 9.1 (SE 0.2); Ca: 7·3 (SE 0.1) and scFOS: 9.1 (SE 0.1) $\log _{10}$ colony-forming units/g faeces). Effects of both $\mathrm{Ca}$ and scFOS on the intestinal microbiota have been shown before ${ }^{(8,13,14,28)}$.

Also, the increase in total permeability caused by dietary scFOS is shown to be localised in the colon. This was hypothesised, as these carbohydrates, or prebiotics, are rapidly hydrolysed and metabolised in the colon by the endogenous microbiota ${ }^{(29)}$. In this way, scFOS change the bacterial composition, which is often assumed to be beneficial ${ }^{(30)}$. In the present study, scFOS were supplemented, which have a degree of polymerisation 
ranging from 2 to 8. Supplementation with long-chain FOS (degree of polymerisation between 2 and 60) alters the microbiota differently from supplementation with scFOS, because of differences in fermentation rate ${ }^{(31)}$ and amount and pattern of SCFA production $^{(32)}$. However, Ten Bruggencate et al. ${ }^{(8)}$ showed that scFOS and inulin (mix of short-chain and long-chain FOS; average degree of polymerisation between 10 and 12) both impaired resistance to intestinal infection in rats. On the contrary, Petersen et al. ${ }^{(33)}$ only observed increased translocation of Salmonella in mice supplemented with scFOS, and not in mice supplemented with inulin. The decreased food intake of the rats fed scFOS might be due to rapid and extensive fermentation of scFOS causing bloating and abdominal cramps ${ }^{(34)}$. These unpleasant feelings decrease appetite. As the permeability marker data are expressed as percentage of intake, the differences in food intake do not influence the outcome measurements. The Cr-EDTA excretion increased gradually with time in the scFOS group. This might reflect relatively slow gut microbiota adaptations due to scFOS. The change in the intestinal permeability by supplemental scFOS has previously been associated with a decreased resistance to intestinal infections ${ }^{(14)}$.

Paracellular permeability in the small intestine, determined by individual lactulose excretion, was not significantly influenced by the supplementation of scFOS to the diet. A similar site-specific effect of scFOS has been observed in previous studies in our laboratory. In one study, dietary scFOS increased mucosal inflammation in the caecum and colon after oral infection with Salmonella enteritidis, but not in the ileum ${ }^{(12)}$. In another study, scFOS supplementation increased mucin concentration and stimulated mucosal lactobacilli and enterobacteria in the caecum and colon, but again not in the ileum $^{(14)}$. scFOS therefore clearly have region-specific effects in rats: in the colon they may exert undesirable effects, while in the small intestine no such effects were observed. Results fit with the idea that the physiological effects of scFOS are microbiota mediated and these mainly reside in the caecum and colon. Furthermore, although fermentation in the rat takes place in both the caecum and colon, the similarity of the effects of scFOS in both these organs supports the use of the rat as an appropriate model for human colonic fermentation.

Interestingly, most adverse effects of scFOS on intestinal infection, which are mentioned in this paper ${ }^{(12-14)}$, were inhibited when Ca was supplemented to the $\operatorname{diet}^{(8)}$. The Ca content of the control diet in these studies and the present investigation matches with the lower limit of the human intake range, while the Ca-supplemented diet provided more than the general habitual dietary Ca intake ${ }^{(11,35)}$. The dose of scFOS used in the present study (6\%) is high, but probably realistic for the human situation. Daily intake of fructosebased non-digestible carbohydrates has been estimated up to $10 \mathrm{~g}^{(36)}$. This corresponds to $2 \%$ in the diet, assuming a total food intake of $500 \mathrm{~g}$ dry weight/d. This estimation does not take into account consumption of specific meals and products supplemented with fructose-based carbohydrates, typically 3-10 g/portion ${ }^{(37)}$. As the human diet still contains other non-digestible carbohydrates, supplementation with $6 \%$ is high, but certainly not unrealistic. When scFOS are fermented, SCFA are produced, which lead to acidification of the intestinal lumen. Subsequently, the protective effect of supplemental Ca might be due to formation of the insoluble calcium phosphate complex in the small intestine. In the colon, solubilisation of this complex increases the buffering 
capacity of the intestinal lumen, which may counteract the adverse effects of acidic fermentation of $\mathrm{scFOS}^{(38)}$. Indeed, the $\mathrm{pH}$ of faecal water of the rats fed the high-Ca diet was higher than that of the rats fed the low-Ca control diet. It will be interesting to investigate the effect on intestinal permeability when both Ca and scFOS are supplemented to the diet. This will be further explored in following studies. Finally, when studying the effects of non-digestible carbohydrates, Ca content of the diet should be taken into account.

Since the individual lactulose and mannitol excretion in urine were not influenced by the different diets, the relevance of the lactulose:mannitol ratio is questionable. Bijlsma et al. $^{\left({ }^{(9)}\right.}$ suggested that the individual lactulose and mannitol excretion values provide more information than the ratio of the two sugars. In fact, mannitol excretion in urine depends mainly on the magnitude of solvent drag caused by villus tip hyperosmolality, while urinary lactulose results truly represent paracellular permeability. By combining the two probes in a ratio, the information is lost and wrong conclusions can be drawn. For example, in the case of coeliac disease, mannitol excretion is clearly decreased because of villus atrophy, while urinary lactulose recovery is increased ${ }^{(40)}$. These findings are more informative than a ratio. In the present study, the ratio indicates that dietary effects exist, while the individual lactulose and mannitol results are not affected by the diet. Based on these findings, we conclude that small-intestinal permeability is not changed by Ca or scFOS.

In the Ussing chamber experiments, we did not observe any effect of the different faecal waters, prepared from faeces of the nutritional study, on permeability. Nevertheless, the $\mathrm{pH}$ of the faecal waters was different due to the diets. This demonstrates that fermentation of scFOS indeed influenced colonic contents, supporting the use of faecal water to study the potential scFOS effects. These Ussing chamber results suggest that the effect of $\mathrm{Ca}$ and scFOS on colonic permeability is not mediated by differences in faecal water composition. Although we observed a change in cytotoxicity of faecal water by Ca and scFOS in previous studies ${ }^{(7,12,13,41)}$, apparently luminal cytotoxicity is not responsible for the effects on colonic permeability. However, it should be kept in mind that, for example, SCFA produced by scFOS fermentation are rapidly absorbed by the colonic mucosa and therefore are largely absent in faecal waters ${ }^{(42)}$, although they might have been capable of influencing the gut wall in vivo and thus the permeability. Additionally, exposure time of the colonic tissue to the faecal water in the Ussing chamber is much shorter than that occurs in vivo. Nevertheless, we hypothesise that other mechanisms, which cannot be mimicked in the Ussing chamber, may play a role in the effects of $\mathrm{Ca}$ and scFOS on permeability. A possible hypothesis is that Ca influences the viscosity of the mucus layer, thereby altering intestinal permeability ${ }^{(43)}$. Handling the colonic tissue for use in the Ussing chambers results in partial loss of this layer, hence this effect may be missed. Moreover, as mentioned earlier and described in other papers, both Ca and scFOS modulate the intestinal microbiota ${ }^{(12,28)}$, and these microbes are not present in the Ussing chambers or in the different faecal waters used in the experiments.

Although extracellular $\mathrm{Ca}$ is crucial for the maintenance of intestinal tight junction function in cell studies ${ }^{(44)}$, no direct effect of Ca on permeability in Ussing chambers was observed when colonic tissue was exposed to Ca-free Ringer solution on the luminal 
side. These differences in observation suggest that the effect of $\mathrm{Ca}$ on permeability as observed in vivo is either indirect or long term or both.

In conclusion, the present study shows that dietary Ca decreases, while scFOS increase the permeability of the rat colon. We hypothesise that modulation of mucins and/or microbiota is important for the permeability effects of both $\mathrm{Ca}$ and scFOS, since in vivo results could not be mimicked in Ussing chambers. The results of the present study emphasise that nutrition can play an important role in the modulation of gut epithelial integrity.

\section{Acknowledgements}

The authors wish to thank the biotechnicians at the Small Animal Centre (Wageningen University and Research Centre, The Netherlands) for their expert assistance.

\section{REFERENCES}

1. Farhadi A, Banan A, Fields J, et al. (2003) Intestinal barrier: an interface between health and disease. J Gastroenterol Hepatol 18, 479-497.

2. Arrieta MC, Bistritz L \& Meddings JB (2006) Alterations in intestinal permeability. Gut 55, 1512-1520.

3. Groschwitz KR \& Hogan SP (2009) Intestinal barrier function: molecular regulation and disease pathogenesis. J Allergy Clin Immunol 124, 3-20; quiz 21-22.

4. Spiller RC (2007) Role of infection in irritable bowel syndrome. J Gastroenterol 42, Suppl. 17, 41-47.

5. Clayburgh DR, Shen L \& Turner JR (2004) A porous defense: the leaky epithelial barrier in intestinal disease. Lab Invest 84, 282-291.

6. Bovee-Oudenhoven IM, Lettink-Wissink ML, Van Doesburg W, et al. (2003) Diarrhea caused by enterotoxigenic Escherichia coli infection of humans is inhibited by dietary calcium. Gastroenterology $125,469-476$.

7. Bovee-Oudenhoven IM, Termont DS, Weerkamp AH, et al. (1997) Dietary calcium inhibits the intestinal colonization and translocation of Salmonella in rats. Gastroenterology 113, 550-557.

8. Ten Bruggencate SJ, Bovee-Oudenhoven IM, Lettink-Wissink ML, et al. (2004) Dietary fructooligosaccharides and inulin decrease resistance of rats to Salmonella: protective role of calcium. Gut 53, $530-535$.

9. Govers MJ, Termont DS, Lapre JA, et al. (1996) Calcium in milk products precipitates intestinal fatty acids and secondary bile acids and thus inhibits colonic cytotoxicity in humans. Cancer Res 56, 32703275.

10. Lapre JA, De Vries HT, Koeman JH, et al. (1993) The antiproliferative effect of dietary calcium on colonic epithelium is mediated by luminal surfactants and dependent on the type of dietary fat. Cancer Res 53, 784-789.

11. Schepens MA, Schonewille AJ, Vink C, et al. (2009) Supplemental calcium attenuates the colitis-related increase in diarrhea, intestinal permeability, and extracellular matrix breakdown in HLA-B27 transgenic rats. J Nutr 139, 1525-1533.

12. Bovee-Oudenhoven IM, Ten Bruggencate SJ, Lettink-Wissink ML, et al. (2003) Dietary fructooligosaccharides and lactulose inhibit intestinal colonisation but stimulate translocation of Salmonella in rats. Gut $52,1572-1578$.

13. Ten Bruggencate SJ, Bovee-Oudenhoven IM, Lettink-Wissink ML, et al. (2003) Dietary fructooligosaccharides dosedependently increase translocation of salmonella in rats. J Nutr 133, 2313-2318.

14. Ten Bruggencate SJ, Bovee-Oudenhoven IM, Lettink-Wissink ML, et al. (2005) Dietary fructooligosaccharides increase intestinal permeability in rats. J Nutr 135, 837-842.

15. Aabakken L (1989) Cr-ethylenediaminetetraacetic acid absorption test. Methodologic aspects. Scand J Gastroenterol 24, 351-358.

16. Meddings JB \& Gibbons I (1998) Discrimination of sitespecific alterations in gastrointestinal permeability in the rat. Gastroenterology 114, 83-92. 
17. Reeves PG, Nielsen FH \& Fahey GC Jr (1993) AIN-93 purified diets for laboratory rodents: final report of the American Institute of Nutrition ad hoc writing committee on the reformulation of the AIN-76A rodent diet. J Nutr 123, 1939-1951.

18. Arslan G, Atasever T, Cindoruk M, et al. (2001) (51)CrEDTA colonic permeability and therapy response in patients with ulcerative colitis. Nucl Med Commun 22, 997-1001.

19. Binnerts W, Van het Klooster A \& Frens A (1968) Soluble chromium indicator measured by atomic absorption in digestion experiments. Vet Rec 82, 470.

20. Bjarnason I (1994) Intestinal permeability. Gut 35, S18-S22.

21. Sesink AL, Termont DS, Kleibeuker JH, et al. (1999) Red meat and colon cancer: the cytotoxic and hyperproliferative effects of dietary heme. Cancer Res 59, 5704-5709.

22. Hansen I, Knudsen KE \& Eggum BO (1992) Gastrointestinal implications in the rat of wheat bran, oat bran and pea fibre. Br J Nutr 68, 451-462.

23. Arnott ID, Kingstone K \& Ghosh S (2000) Abnormal intestinal permeability predicts relapse in inactive Crohn disease. Scand J Gastroenterol 35, 1163-1169.

24. Wyatt J, Vogelsang H, Hubl W, et al. (1993) Intestinal permeability and the prediction of relapse in Crohn's disease. Lancet 341, 1437-1439.

25. Sartor RB (2006) Mechanisms of disease: pathogenesis of Crohn's disease and ulcerative colitis. Nat Clin Pract Gastroenterol Hepatol 3, 390-407.

26. Guarner F \& Malagelada JR (2003) Gut flora in health and disease. Lancet 361, 512-519.

27. Mangell P, Nejdfors P, Wang M, et al. (2002) Lactobacillus plantarum 299v inhibits Escherichia coliinduced intestinal permeability. Dig Dis Sci 47, 511-516.

28. Bovee-Oudenhoven IM, Wissink ML, Wouters JT, et al. (1999) Dietary calcium phosphate stimulates intestinal lactobacilli and decreases the severity of a Salmonella infection in rats. J Nutr 129, 607-612.

29. Andersson HB, Ellegard LH \& Bosaeus IG (1999) Nondigestibility characteristics of inulin and oligofructose in humans. J Nutr 129, 1428S-1430S.

30. Gibson GR \& Roberfroid MB (1995) Dietary modulation of the human colonic microbiota: introducing the concept of prebiotics. J Nutr 125, 1401-1412.

31. Roberfroid MB, Van Loo JA\&GibsonGR(1998) The bifidogenic nature of chicory inulin and its hydrolysis products. J Nutr 128, 11-19.

32. Macfarlane S \& Macfarlane GT (2003) Regulation of shortchain fatty acid production. Proc Nutr Soc 62, 67-72.

33. Petersen A, Heegaard PM, Pedersen AL, et al. (2009) Some putative prebiotics increase the severity of Salmonella enterica serovar Typhimurium infection in mice. BMC Microbiol 9, 245.

34. Briet F, Achour L, Flourie B, et al. (1995) Symptomatic response to varying levels of fructooligosaccharides consumed occasionally or regularly. Eur J Clin Nutr 49, 501-507.

35. AlaimoK, McDowell MA, Briefel RR, et al. (1994) Dietary intake of vitamins, minerals, and fiber of persons ages 2 months and over in the United States: Third National Health and Nutrition Examination Survey, Phase 1, 1988-91. Adv Data, 1-28.

36. Coussement PA (1999) Inulin and oligofructose: safe intakes and legal status. J Nutr 129, 1412S-1417S.

37. van Loo J, Coussement P, de Leenheer L, et al. (1995) On the presence of inulin and oligofructose as natural ingredients in the western diet. Crit Rev Food Sci Nutr 35, 525-552.

38. Remesy C, Levrat MA, Gamet L, et al. (1993) Cecal fermentations in rats fed oligosaccharides (inulin) are modulated by dietary calcium level. Am J Physiol 264, G855-G862.

39. Bijlsma PB, Peeters RA, Groot JA, et al. (1995) Differential in vivo and in vitro intestinal permeability to lactulose and mannitol in animals and humans: a hypothesis. Gastroenterology 108, 687-696.

40. Ukabam SO \& Cooper BT (1984) Small intestinal permeability to mannitol, lactulose, and polyethylene glycol 400 in celiac disease. Dig Dis Sci 29, 809-816.

41. Govers MJ \& Van der Meer R (1993) Effects of dietary calcium and phosphate on the intestinal interactions between calcium, phosphate, fatty acids, and bile acids. Gut 34, 365-370.

42. Alles MS, Hautvast JG, Nagengast FM, et al. (1996) Fate of fructo-oligosaccharides in the human intestine. Br J Nutr 76, 211-221.

43. Crowther RS, Marriott C \& James SL (1984) Cation induced changes in the rheological properties of purified mucus glycoprotein gels. Biorheology 21, 253-263.

44. Gonzalez-Mariscal L, Contreras RG, Bolivar JJ, et al. (1990) Role of calcium in tight junction formation between epithelial cells. Am J Physiol 259, C978-C986. 
Chapter 5

1 Chapter 5 


\section{The protective effect of supplemental calcium on colonic permeability depends on a calcium-phosphate-induced increase in luminal buffering capacity}

Marloes A. A. Schepens Sandra J.M. ten Bruggencate Arjan J. Schonewille Robert-Jan M. Brummer Roelof van der Meer Ingeborg M. J. Bovee-Oudenhoven 


\section{Abstract}

Objective: An increased intestinal permeability is associated with several diseases. Previously, we have shown that dietary calcium decreases colonic permeability in rats. This might be explained by a calcium-phosphate-induced increase in luminal buffering capacity, which protects against an acidic $\mathrm{pH}$ due to microbial fermentation. Therefore, we investigated whether dietary phosphate is a co-player in the effect of calcium on permeability.

Methods: Rats were fed a humanized low-calcium diet, or a similar diet supplemented with calcium and containing either high, medium or low phosphate concentrations. Chromium-EDTA was added as an inert dietary intestinal permeability marker. After dietary adaptation, short-chain fructo-oligosaccharides (scFOS) were added to all diets to stimulate fermentation, acidify the colonic contents and induce an increase in permeability.

Results: Dietary calcium prevented the scFOS-induced increase in intestinal permeability in rats fed medium- and high-phosphate levels but not in those fed the low-phosphate diet. This was associated with higher faecal water cytotoxicity and higher caecal lactate levels in the latter group. Moreover, food intake and body weight during scFOS supplementation were adversely affected by the low-phosphate diet. Importantly, luminal buffering capacity was higher in rats fed the medium- and highphosphate diets compared to rats fed the low-phosphate diet.

Conclusion: The protective effect of dietary calcium on intestinal permeability is impaired if dietary phosphate is low. This is associated with a calcium-phosphateinduced increase in luminal buffering capacity. Dragging phosphate into the colon and thereby increasing the colonic phosphate concentration, is at least part of the mechanism behind the protective effect of calcium on intestinal permeability. 


\section{INTRODUCTION}

The intestinal tract is lined by a monolayer of epithelial cells, which is the largest and most important barrier between the body's internal milieu and the hostile external environment. Tight contact between the epithelial cells prevents access of luminal digestive enzymes, toxins, antigens and enteric microbiota to underlying tissue compartments ${ }^{(1-3)}$. Intestinal barrier dysfunction is suggested to be associated with the pathogenesis of a variety of intestinal diseases, including inflammatory bowel disease, coeliac disease, post-infectious irritable bowel syndrome and food allergy ${ }^{(1-5)}$.

Interestingly, dietary components can influence the epithelial barrier, in particular intestinal permeability, and hence possibly modulate disease development. We are interested in the effect of dietary calcium, since it has been shown in several controlled studies that calcium is important for protection against intestinal infections with foodborne bacterial pathogens, both in rats and in humans $s^{(6-8)(T e n ~ B r u g g e n c a t e ; ~ a c c e p t e d ~ f o r ~ p u b l i c a t i o n, ~}$ Br Jutr 2010). In addition, calcium displayed cytoprotective effects in several studies in the field of colon carcinogenesis by precipitating cytotoxic surfactants, such as secondary bile acids $s^{(9,10)}$. Furthermore, supplemental calcium attenuated the development of colitis in HLA-B27 transgenic rats, which was associated with the prevention of a colitis-related increase in intestinal permeability due to calcium ${ }^{(11)}$. In a previous study, we identified that the effect of dietary calcium on intestinal permeability is located in the colon ${ }^{(12)}$. At present, however, it is still not clear how calcium exerts its protective effect on colonic permeability. Upon dietary intake, an insoluble calcium phosphate complex is formed in the small intestine, both in rats ${ }^{(13)}$ and in humans ${ }^{(14)}$. In this way, calcium prevents phosphate from being absorbed in the small intestine, and drags phosphate into the colon. In the colon, solubilisation of this complex increases the buffering capacity of the luminal contents, which protects the intestinal mucosa from being injured by an acidic $\mathrm{pH}$ due to microbial fermentation ${ }^{(15)}$. This mechanism might play a role in the effect of calcium on intestinal permeability and it implies that dietary phosphate intake might be of importance for the effect of calcium on permeability.

In the present study we investigated the potential effect of dietary phosphate on calcium-induced lowering of intestinal permeability, by applying high, medium or low phosphate concentrations in a high-calcium diet. Inert chromium-EDTA (CrEDTA) was added to the diets as an established marker for intestinal permeability ${ }^{(16,17)}$. After dietary adaptation for 10 days, short-chain fructo-oligosaccharides (scFOS) were introduced in the diets. The intestinal fermentation of scFOS leads to a luminal organic acid-load, which challenges the buffering capacity. We hypothesise that intestinal permeability will increase on a high-calcium diet with concomitant low dietary phosphate levels because of a decreased buffering capacity.

\section{MATERIALS AND METHODS}

\section{Experimental design: animals and diets}

The experimental protocol was approved by the animal welfare committee of 
Wageningen University and Research Centre (Wageningen, The Netherlands). Specific pathogen-free outbred male Wistar rats (WU, Harlan, Horst, The Netherlands), 8 weeks old and with a mean body weight of $288 \mathrm{~g}$ at the start of the experiment, were housed individually in metabolic cages. Animals were kept in a temperature- and humiditycontrolled environment in a 12-h light-dark cycle. Rats ( $\mathrm{n}=10$ per dietary group) were fed a purified 'humanised' Western diet in restricted quantities (16 g/day). Restricted feeding was necessary to prevent scFOS-induced differences in food consumption as observed earlier ${ }^{(18)}$ and hence differences in vitamin, mineral and CrEDTA intake. Demineralised drinking water was supplied ad libitum. The reference diet (lowcalcium, medium-phosphate; LCaMP), which has been used as a reference diet in our previous experiments ${ }^{(11,12)}$, contained (per kg): $200 \mathrm{~g}$ acid casein, $326 \mathrm{~g}$ corn starch, 172 g glucose, 160 g palm oil, 40 g corn oil, 50 g cellulose, 2 g CrEDTA (see below) and 5.16 g CaHPO $42 \mathrm{H}_{2} \mathrm{O}$ (corresponding to $30 \mathrm{mmol}$ calcium/kg diet; Sigma-Aldrich, St Louis, MO). Vitamins and minerals (other than calcium) were added to the diets according to AIN93 ${ }^{(19)}$. The concentration of vitamins and minerals was increased by $20 \%$ to ensure adequate intake during restricted feeding. The diets had a high fat content to mimic the composition of a Western human diet. Dietary phosphate mainly originates from calcium phosphate and the protein source of the diet (casein: about $40 \mathrm{mmol} \mathrm{PO} / 4 / \mathrm{kg}$ diet). The experimental diets were supplemented with $90 \mathrm{mmol} \mathrm{CaHPO} \cdot 2 \mathrm{H}_{2} \mathrm{O} / \mathrm{kg}$ diet (high-calcium, high-phosphate; $\mathrm{HCaHP}$ ), or with $90 \mathrm{mmol} \mathrm{CaCl}_{2} \cdot 2 \mathrm{H}_{2} \mathrm{O} / \mathrm{kg}$ diet (highcalcium, medium-phosphate; HCaMP), at the expense of glucose. The high-calcium lowphosphate diet (HCaLP) was supplemented with $90 \mathrm{mmol} \mathrm{CaCl}_{2} \cdot 2 \mathrm{H}_{2} \mathrm{O} / \mathrm{kg}$ diet, and the casein of this diet was replaced by whey protein isolate as a low-phosphate protein source (BiPRO, Davisco Foods International, Inc, MN; about $5 \mathrm{mmol} \mathrm{PO}_{4} / \mathrm{kg}$ diet). Thus, the experiment consisted of four different diets: LCaMP diet (reference diet; $30 \mathrm{mmol}$ calcium and $70 \mathrm{mmol}$ phosphate per kg diet ), HCaHP diet (positive control since this diet has been used in our previous experiments, showing effects of calcium on intestinal permeability ${ }^{(11,12)} ; 120 \mathrm{mmol}$ calcium and $160 \mathrm{mmol}$ phosphate per $\mathrm{kg}$ diet), HCaMP diet (120 mmol calcium and $70 \mathrm{mmol}$ phosphate per $\mathrm{kg}$ diet) and HCaLP diet (120 mmol calcium and $35 \mathrm{mmol}$ phosphate per $\mathrm{kg}$ diet). Inert CrEDTA was added to all diets to quantify intestinal permeability ${ }^{(17)}$. CrEDTA solution was prepared as described elsewhere and subsequently freeze-dried ${ }^{(20)}$. To check the complete formation and stability of the CrEDTA complex, the prepared CrEDTA solution was passed through a cation-exchange resin column (Chelex 100 Resin; Bio-Rad, Hercules, CA). No uncomplexed $\mathrm{Cr}^{3+}$ ions were present. Rats were fed the experimental diets for 10 days, after which all diets were supplemented with $60 \mathrm{~g} \mathrm{scFOS} / \mathrm{kg} \operatorname{diet}(6 \% \mathrm{w} / \mathrm{w}$; Raftilose ( $\mathrm{P} 95$, Orafti, Tienen) at the expense of glucose. Food intake was recorded daily and animal weight twice every week. At experimental day 20, rats were anaesthetised with isoflurane and killed. The caecum was excised and caecal contents were collected.

\section{Measurement of intestinal permeability}

Total $24 \mathrm{~h}$ urine samples were collected at experimental day 10 and 20. For CrEDTA measurement, urine was acidified with $50 \mathrm{~g} / \mathrm{L}$ trichloroacetic acid, centrifuged for $2 \mathrm{~min}$ at $14,000 \mathrm{~g}$ and the supernatant was subsequently diluted with $0.5 \mathrm{~g} / \mathrm{L} \mathrm{CsCl}$. 
Then, chromium was analysed by inductively coupled plasma-atomic emission spectrophotometry (ICP-AES).

\section{Analyses of faeces and caecal contents}

All faeces were collected during the last 3 days of experimental feeding without scFOS (pre-scFOS; experimental days 8, 9 and 10), and during the last 3 days of the scFOSsupplementation period (experimental days 18,19 and 20). Faeces and caecal contents were freeze-dried and subsequently ground to obtain homogeneous powdered samples. Calcium and phosphorus were quantified in faeces of the pre-scFOS period. To this end, faeces were treated with $50 \mathrm{~g} / \mathrm{L}$ trichloroacetic acid, centrifuged for $2 \mathrm{~min}$ at $14,000 \mathrm{~g}$, diluted with $0.5 \mathrm{~g} / \mathrm{L} \mathrm{CsCl}$, and analysed by ICP-AES. The buffering capacity was also determined in faeces of the pre-scFOS period. To this end, pools of freezedried faeces were reconstituted with double-distilled water to $15 \%$ dry weight. The quantities of hydrochloric acid required to decrease the $\mathrm{pH}$ to 5 in these samples was measured, as described earlier ${ }^{(15)}$. For the cytotoxicity assay, faecal water was prepared by reconstituting freeze-dried faeces with double-distilled water to $25 \%$ dry weight as previously described ${ }^{(21)}$. Cytotoxicity of faecal water of the scFOS-supplementation period was determined by potassium release of a human erythrocyte suspension after incubation with faecal water, as described earlier ${ }^{(22)}$, and validated earlier with intestinal epithelial cells ${ }^{(23)}$. Cytotoxicity was calculated and expressed as a percentage of maximal lysis. Total lactic acid was determined in caecal contents by using a colorimetric enzymatic kit (Enzyplus; BioControl Systems, Inc., Bellevue, WA), as described earlier ${ }^{(24)}$.

\section{Statistical analysis}

All results are expressed as mean \pm SEM. The predefined comparisons of interest were the LCaMP diet versus the HCaHP diet to study the effect of calcium (these two diets have been compared in our previous studies, showing effects of calcium on intestinal permeability ${ }^{(11,12)}$ ), and the HCaHP diet versus the HCaMP diet, and the HCaMP diet versus the HCaLP diet to study phosphate effects. Statistics were done by using oneway ANOVA or Kruskal-Wallis, depending on normality of the data. If differences were significant, this was followed by Student's t test (for normally distributed data) or Mann-Whitney U test (for non-normally distributed data) to identify the significant dietary effects. Differences were considered statistically significant when $\mathrm{P}<0.05$ (all two-sided). Statistical analyses were conducted with GraphPad Prism version 5.01 (GraphPad Software, Inc., La Jolla, CA).

\section{RESULTS}

\section{Animal growth and food intake}

No diet-induced differences in animal growth and food intake were observed before supplementation with scFOS: all rats consumed the provided $16 \mathrm{~g} /$ day as intended. However,animal growth was affected by the different diets during scFOS supplementation. Rats from the HCaLP group gained less weight during supplementation with scFOS than 

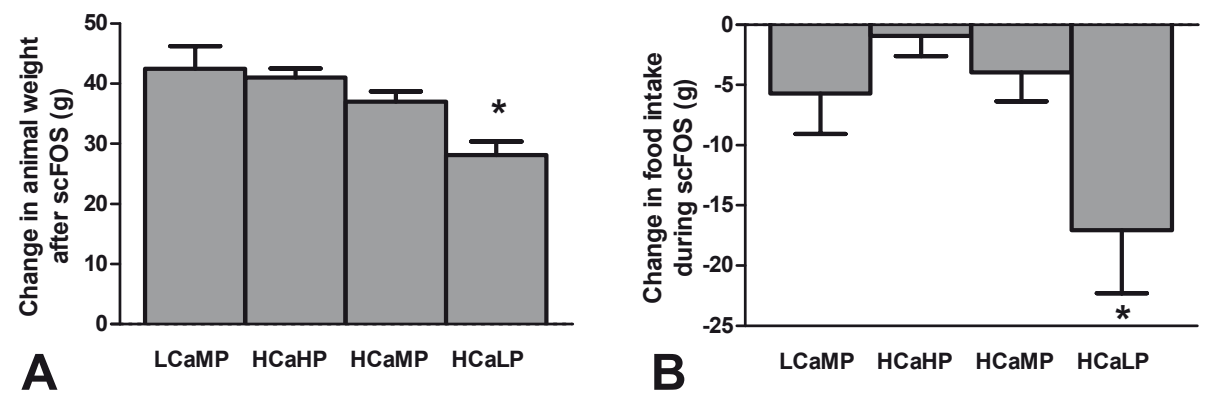

Figure 1: Effect of dietary calcium and phosphate on body weight gain (A) and food intake (B) during shortchain fructo-oligosaccharides (scFOS) supplementation ( $n=10 /$ group). (A) Results are expressed as mean (SEM) change in body weight at the end of the experiment (experimental day 20) compared to body weight just before scFOS supplementation started (experimental day 9). (B) Results are expressed as the cumulative change in food intake (mean \pm SEM) during scFOS supplementation compared to the food consumption before scFOS supplementation. *Different compared to rats on HCaMP diet (Figure $1 A, P=0.006$; Figure 1B, $P=0.02$ ). LCaMP, low-calcium medium-phosphate; HCaHP, high-calcium high-phosphate; HCaMP, high-calcium mediumphosphate; HCaLP, high-calcium low-phosphate.

rats from the HCaMP group (Figure $1 \mathrm{~A}, \mathrm{P}=0.006$ ). The other dietary groups did not differ with respect to growth. The results for food intake were similar: HCaLP-fed rats had a more decreased food consumption than rats from the HCaMP group during scFOS supplementation (Figure 1B, $\mathrm{P}=0.02$ ).

\section{Faecal baseline characteristics before scFOS supplementation}

Daily output of faeces, based on dry weight, was increased due to calcium supplementation (Table $1 ; \mathrm{P}<0.0001$ ), in accordance with previous work ${ }^{(7,13)}$. To check whether the dietary interventions indeed affected the baseline calcium and phosphate levels in the colonic lumen before scFOS supplementation, we measured total calcium and phosphorus in faeces (Table 1). Indeed the calcium concentration was clearly higher in the calciumsupplemented group (HCaHP: $\mathrm{P}<0.0001)$ compared to levels in the LCaMP group. Phosphorus levels in faeces also corresponded well with the dietary intervention. Faecal phosphorus was increased in the rats fed the HCaHP diet $(\mathrm{P}<0.0001)$ compared to the rats fed the LCaMP diet. As expected, the concentration of phosphorus was decreased in the HCaMP group $(\mathrm{P}<0.0001)$ compared to the HCaHP group, and further diminished in the rats fed the HCaLP diet compared to the HCaMP group $(\mathrm{P}<0.0001)$. Subsequently, the buffering capacity of these faecal samples was determined to evaluate whether the diets affected this capacity as hypothesised. Luminal buffering capacity was higher in the HCaHP group $(\mathrm{P}<0.0001)$ in comparison with the rats fed the LCaMP diet (Figure 2). Also as expected, buffering capacity of the faeces was lower in rats on the HCaMP $\operatorname{diet}(\mathrm{P}=0.01)$ compared to rats from the HCaHP group. And indeed, buffering capacity was further decreased in the rats fed the HCaLP diet $(\mathrm{P}=0.04)$ compared to rats fed the HCaMP diet. The results of the faecal buffering capacity measurements after scFOS supplementation showed similar dietary effects (data not shown). 
Table 1: Effect of diet on faecal baseline characteristics before short-chain fructo-oligosaccharides supplementation

\begin{tabular}{lcccc}
\hline & LCaMP & HCaHP & HCaMP & HCaLP \\
\hline Faecal dry weight $(\mathrm{g} / \mathrm{d})$ & $1.02 \pm 0.02$ & $1.31 \pm 0.02^{\#}$ & $1.37 \pm 0.03$ & $1.38 \pm 0.04$ \\
Calcium excretion $(\mu \mathrm{mol} / \mathrm{d})$ & $78 \pm 10$ & $1051 \pm 34^{\#}$ & $1098 \pm 19$ & $887 \pm 19^{*}$ \\
Phosphorus excretion $(\mu \mathrm{mol} / \mathrm{d})$ & $65 \pm 6$ & $733 \pm 21^{\#}$ & $350 \pm 17^{\dagger}$ & $116 \pm 8^{*}$ \\
\hline
\end{tabular}

Results are expressed as mean (SEM) ( $n=10 /$ group). "Different compared to rats on LCaMP diet $(P<0.0001)$. ${ }^{+}$Different compared to rats on HCaHP diet $(P<0.0001)$. *Different compared to rats on HCaMP $\operatorname{diet}(P<0.0001)$. LCaMP, low-calcium medium-phosphate; HCaHP, high-calcium high-phosphate; HCaMP, high-calcium mediumphosphate; HCaLP, high-calcium low-phosphate.

\section{The effect of dietary calcium and phosphate on intestinal permeability}

The main outcome of the present study is the dietary effect on intestinal permeability, measured by urinary CrEDTA excretion. Dietary calcium already decreased intestinal permeability before the colon was challenged with scFOS; however, this was not the case in the rats fed the HCaLP diet. Urinary CrEDTA expressed as percentage of dietary intake was $5.9 \pm 0.5$ in the LCaMP group; $4.2 \pm 0.3$ in the HCaHP group $(\mathrm{P}=0.007$, compared to LCaMP); $4.1 \pm 0.2$ in the HCaMP group; and $6.5 \pm 0.5$ in the HCaLP group $(\mathrm{P}=0.0002$ compared to HCaMP). Importantly, after scFOS supplementation, dietary calcium only prevented the fermentation-induced increase in intestinal permeability when rats were fed a diet containing high or medium levels of phosphate (Figure 3, HCaHP, $\mathrm{P}=0.049$ compared to LCaMP), but not when rats were fed the low phosphate $\operatorname{diet}$ (Figure 3, $\mathrm{P}=0.04$ compared to HCaMP).

\section{Dietary effects on caecal lactate levels}

To investigate whether fermentation of scFOS is disturbed due to acid accumulation, lactate was quantified in caecal contents ${ }^{(25,26)}$. Calcium supplementation in a highphosphate background (HCaHP) resulted in significantly lower lactate levels in caecal contents compared to rats fed the LCaMP diet (Figure 4A, P=0.006). Lactate levels were similar in the HCaMP group compared to rats fed the HCaHP diet, while these levels were higher in rats on the HCaLP diet compared to the HCaMP group (Figure 4A, $\mathrm{P}=0.01)$. Interestingly, lactate levels are correlated with the colonic permeability results (Spearman $\mathrm{r}=0.45, \mathrm{P}=0.006$ ). The dietary effects on caecal $\mathrm{pH}$ show comparable results, since the $\mathrm{pH}$ in caecum of the HCaHP group was significantly higher than the $\mathrm{pH}$ of the caecal contents of the LCaMP-fed rats ( $6.9 \pm 0.2$ versus $5.8 \pm 0.1$, respectively, $\mathrm{P}=0.0008)$. No differences in caecal pH were observed in the other dietary groups (HCaMP, $7.2 \pm$ 0.2 ; HCaLP, $6.8 \pm 0.3$ ).

\section{Dietary calcium and phosphate influence luminal cytotoxicity}

To investigate whether faecal water cytotoxicity, reflecting colonic mucosal exposure to luminal irritants, plays a role in the dietary effects on colonic permeability, the cytotoxicity assay was performed with faecal water from the period after scFOS 


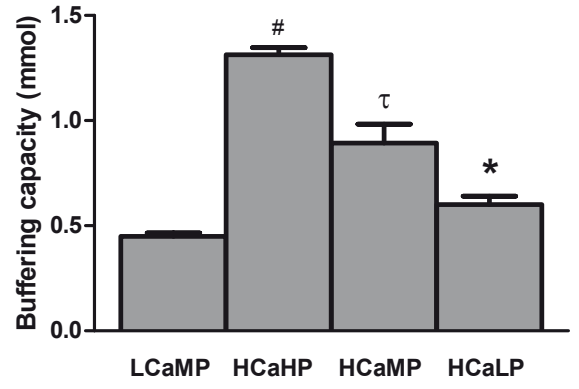

Figure 2: Effect of dietary calcium and phosphate on faecal buffering capacity before short-chain fructo-oligosaccharides supplementation ( $n=10$ ) group). Results from triplicate measurements of pooled faecal waters, expressed as mean (SEM). Buffering capacity is calculated as the quantities of hydrochloric acid (in mmol) required to decrease the $p H$ to 5. "Different compared to rats on LCaMP diet $(P<0.0001)$. TDifferent compared to rats on HCaHP diet $(P=0.01)$. ${ }^{*}$ Different compared to rats on HCaMP diet $(P=0.04)$. LCaMP, low-calcium mediumphosphate; HCaHP, high-calcium high-phosphate; HCaMP, high-calcium medium-phosphate; HCaLP, high-calcium low-phosphate.

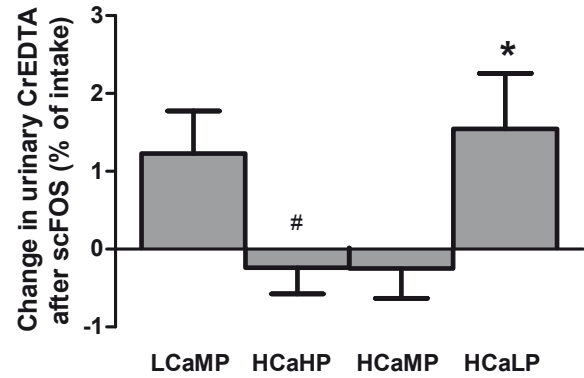

Figure 3: Effect of dietary calcium and phosphate on urinary CrEDTA excretion, a marker for intestinal paracellular permeability, after short-chain fructooligosaccharides (scFOS) supplementation ( $n=10$ ) group). Results are expressed as mean (SEM) change in urinary CrEDTA excretion (\% of intake) at the end of the experiment (experimental day 20) compared to urinary CrEDTA excretion (\% of intake) before scFOS supplementation started (experimental day 10). "Different compared to rats on LCaMP $\operatorname{diet}(P=0.049)$. *Different compared to rats on HCaMP diet $(P=0.04)$. LCaMP, low-calcium mediumphosphate; HCaHP, high-calcium high-phosphate; HCaMP, high-calcium medium-phosphate; HCaLP, high-calcium low-phosphate.

supplementation. Cytotoxic activity of faecal water was lower in rats fed the HCaHP diet (Figure $4 \mathrm{~B}, \mathrm{P}=0.006$ ) compared to the rats on the LCaMP diet. In HCaLP-fed rats, luminal cytotoxicity was increased compared to rats fed the HCaMP diet (Figure 4B, P=0.01).

\section{DISCUSSION}

The present study shows that the beneficial effect of dietary calcium on colonic permeability is impaired if dietary phosphate levels are low. If dietary phosphate is sufficient, calcium decreases colonic permeability, which is associated with an increase in luminal buffering capacity. The protective effect of calcium on permeability is also related to lower caecal lactate levels and a decreased faecal cytotoxicity during scFOS supplementation. Besides the abrogation of the protective effect of a high-calcium diet on colonic permeability, low phosphate intake also adversely affected food intake and animal weight after scFOS supplementation.

The protective effect of dietary calcium on intestinal permeability has been shown consistently in several studies using different animal models. It is of relevance that calcium can improve intestinal permeability both in a healthy situation ${ }^{(12)}$, as well as during intestinal infection (Ten Bruggencate; accepted for publication, BrJ Nutr 2010) and colitis development ${ }^{(11)}$. In the latter two studies, the prevention of an increase in intestinal permeability by 

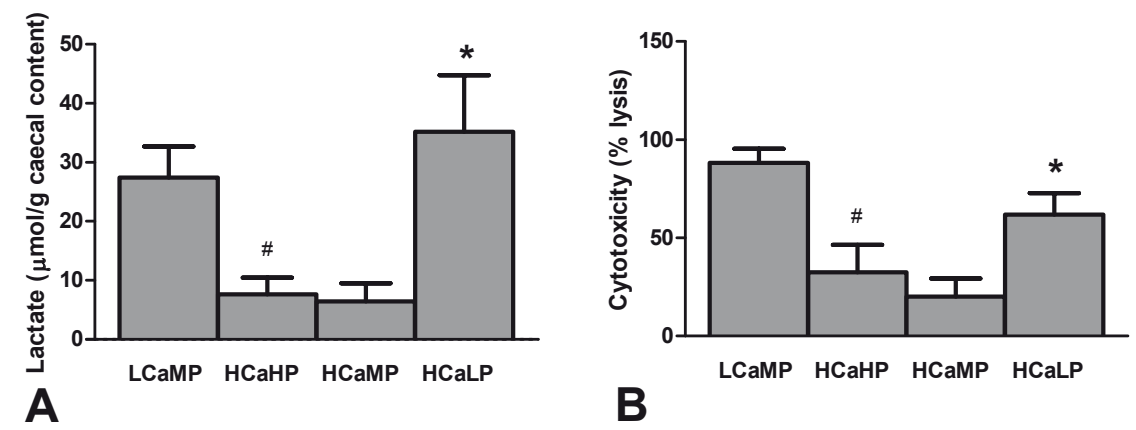

Figure 4: Effect of dietary calcium and phosphate on caecal lactate levels and luminal cytotoxicity after shortchain fructo-oligosaccharides (scFOS) supplementation ( $n=10 /$ group). Results are expressed as mean (SEM) for (A) caecal lactate levels quantified by using a colorimetric enzymatic kit, and for (B) luminal cytotoxicity determined with an erythrocyte bioassay. "Different compared to rats on LCaMP diet $(P=0.006)$. *Different compared to rats on HCaMP diet $(P=0.01)$. LCaMP, low-calcium medium-phosphate; HCaHP, high-calcium highphosphate; HCaMP, high-calcium medium-phosphate; HCaLP, high-calcium low-phosphate.

calcium was associated with an enhanced resistance to the development of intestinal disease. The present study emphasises the potential of calcium to decrease intestinal permeability in a healthy condition; however, it also shows that dietary phosphate intake has to be taken into account.

Since it is still unknown how calcium exerts its beneficial effect on gut permeability, we aimed to investigate whether a calcium-phosphate-induced increase in luminal buffering capacity is involved. By acidifying the colon with scFOS in this study, the capacity to buffer the intestinal contents becomes even more important. Interestingly, scFOS induce an increase in intestinal permeability ${ }^{(12,27)}$, but it is not proven that this is associated with the luminal acidification caused by scFOS, although this seems likely. There are indications from in vitro studies that the extracellular $\mathrm{pH}$ is important for paracellular permeability $^{(28-30)}$. We aimed to show experimental support for the hypothesised effect that the luminal buffering capacity affects intestinal permeability, and is part of the protective effect of calcium, in a situation of enhanced luminal acidification through scFOS in the diet. Indeed, the protective effect of calcium on colonic permeability was impaired if dietary phosphate levels were low and this effect was associated with a decrease of the luminal buffering capacity. Fermentation already starts in the caecum of rats; however, as has been described before ${ }^{(12)}$, it clearly continues in the rat colon, showing the relevance of faecal sampling. These results show that the protective effect of dietary calcium is at least partly due to its role as a carrier of phosphate into the colon. Subsequently, phosphate is responsible for the increase in the colonic buffering capacity.

Calcium supplementation in combination with high or medium dietary phosphate prevented a FOS-induced accumulation of lactate in caecal contents, which was associated with the decrease in intestinal permeability. During rapid fermentation of easily fermentable carbohydrates, lactate accumulation can occur when microorganisms 
that utilise lactate are inhibited ${ }^{(25)}$. These microorganisms might be inhibited by an acidic $\mathrm{pH}$ due to a compromised buffering capacity. It is therefore likely that alterations in microbiota composition or activity are responsible for the changes in caecal lactate levels. Interestingly, intestinal microorganisms, for example Escherichia coli, are able to induce an increase in intestinal permeability ${ }^{(31)}$. Therefore, buffering capacity might also indirectly influence intestinal permeability by modulating the gut microbiota.

An alternative explanation for the dietary effects on intestinal permeability, besides modulation of luminal buffering capacity, might be the influence of supplemental calcium on luminal cytotoxicity. The present study demonstrates that a lack of phosphate counteracts the inhibiting effect of calcium on cytotoxicity, which has been shown in earlier studies ${ }^{(32)}$. Clearly, both calcium and phosphate are needed to precipitate cytotoxic components, which can irritate the intestinal epithelium ${ }^{(33)}$ and subsequently modify permeability. Modulation of luminal cytotoxicity might also change the microbiota, which can thereby exert influence on epithelial integrity ${ }^{(31)}$. We have shown before that calcium affects the gut microbiota ${ }^{\text {(Ten Bruggencate; accepted for publication, } \mathrm{Br}}$ J Nutr 2010)(12,34). The results of the present study suggest that phosphate is necessary for the effect of calcium, but not determinative, i.e. as long as phosphate intake is sufficient ( $\geq 70 \mathrm{mmol} / \mathrm{kg}$ diet), the effect of calcium is not dependent on phosphate.

Impairment of the gut mucosal barrier by scFOS has been shown before in association with a decreased resistance to intestinal infection ${ }^{(18,27,35)}$. These harmful effects were decreased when calcium was supplemented to the diet ${ }^{(8)}$. The present study emphasises again that it is important to take care of a sufficient calcium intake when non-digestible carbohydrates are consumed. Furthermore, the results of this study show that dietary phosphate levels should also be taken into consideration. The adverse effects of low phosphate intake on food consumption and animal weight, which emerged during scFOS supplementation, support this observation.

The calcium and phosphate content of the rat diets in the present study is nutritionally relevant for the human diet. In general, human dietary calcium intake in the Western world ranges from 600-1100 mg daily ${ }^{(36)}$. The calcium concentration of the low-calcium diet corresponds to a daily calcium intake of $600 \mathrm{mg}$ in humans, while the calciumsupplemented diets provided more than the general habitual dietary calcium intake (comparable to $2.4 \mathrm{~g}$ daily), which is not unrealistic when taking calcium supplements ${ }^{(11)}$. In addition, the animal diets contained phosphate levels of approximately $160 \mathrm{mmol} /$ $\mathrm{kg}$ diet (high-phosphate), $70 \mathrm{mmol} / \mathrm{kg}$ diet (medium-phosphate) and $35 \mathrm{mmol} / \mathrm{kg}$ diet (low-phosphate), which are in the range of human intake of about $40 \mathrm{mmol}$ phosphate daily ( $80 \mathrm{mmol} / \mathrm{kg} \mathrm{diet)}$, assuming that humans have a daily dry food intake of about $500 \mathrm{~g}^{(36)}$.

In conclusion, the present study shows that the protective effect of dietary calcium on intestinal permeability is impaired if phosphate levels are low, and this is associated with effects on the luminal buffering capacity. This is particularly important when consuming a diet with non-digestible and rapidly fermentable carbohydrates like scFOS. So, phosphate levels should be sufficient in the diet to accomplish the protective effect of calcium on intestinal permeability. It cannot be excluded that changes in luminal buffering capacity also indirectly modulate intestinal permeability, e.g. by effects on the 
intestinal microbiota. The present study encourages follow-up studies, particularly in humans, to further explore and apply the potential to modulate gut barrier integrity by relatively simple dietary interventions.

\section{Acknowledgements}

The authors wish to thank the biotechnicians at the Small Animal Centre (Wageningen University and Research Centre, The Netherlands) for expert assistance.

\section{REFERENCES}

1. Arrieta MC, Bistritz L \& Meddings JB (2006) Alterations in intestinal permeability. Gut 55, 1512-1520.

2. Groschwitz KR \& Hogan SP (2009) Intestinal barrier function: molecular regulation and disease pathogenesis. J Allergy Clin Immunol 124, 3-20; quiz 21-22.

3. Turner JR (2009) Intestinal mucosal barrier function in health and disease. Nat Rev Immunol 9, 799809.

4. Farhadi A, Banan A, Fields J et al. (2003) Intestinal barrier: an interface between health and disease. J Gastroenterol Hepatol 18, 479-497.

5. Spiller RC (2007) Role of infection in irritable bowel syndrome. J Gastroenterol 42 Suppl 17, 41-47.

6. Bovee-Oudenhoven IM, Lettink-Wissink ML, Van Doesburg W et al. (2003) Diarrhea caused by enterotoxigenic Escherichia coli infection of humans is inhibited by dietary calcium. Gastroenterology $125,469-476$.

7. Bovee-Oudenhoven IM, Termont DS, Weerkamp AH et al. (1997) Dietary calcium inhibits the intestinal colonization and translocation of Salmonella in rats. Gastroenterology 113, 550-557.

8. Ten Bruggencate SJ, Bovee-Oudenhoven IM, Lettink-Wissink ML et al. (2004) Dietary fructooligosaccharides and inulin decrease resistance of rats to salmonella: protective role of calcium. Gut 53, 530-535.

9. Govers MJ, Termont DS, Lapre JA et al. (1996) Calcium in milk products precipitates intestinal fatty acids and secondary bile acids and thus inhibits colonic cytotoxicity in humans. Cancer Res 56, 3270-3275.

10. Lapre JA, De Vries HT, Koeman JH et al. (1993) The antiproliferative effect of dietary calcium on colonic epithelium is mediated by luminal surfactants and dependent on the type of dietary fat. Cancer Res 53, 784-789.

11. Schepens MA, Schonewille AJ, Vink C et al. (2009) Supplemental calcium attenuates the colitis-related increase in diarrhea, intestinal permeability, and extracellular matrix breakdown in HLA-B27 transgenic rats. J Nutr 139, 1525-1533.

12. Schepens MA, Rijnierse A, Schonewille AJ et al. (2010) Dietary calcium decreases but short-chain fructooligosaccharides increase colonic permeability in rats. Br J Nutr, 1-7.

13. Govers MJ \& Van der Meer R (1993) Effects of dietary calcium and phosphate on the intestinal interactions between calcium, phosphate, fatty acids, and bile acids. Gut 34, 365-370.

14. Van der Meer R, Welberg JW, Kuipers F et al. (1990) Effects of supplemental dietary calcium on the intestinal association of calcium, phosphate, and bile acids. Gastroenterology 99, 1653-1659.

15. Remesy C, Levrat MA, Gamet L et al. (1993) Cecal fermentations in rats fed oligosaccharides (inulin) are modulated by dietary calcium level. Am J Physiol 264, G855-862.

16. Aabakken L (1989) Cr-ethylenediaminetetraacetic acid absorption test. Methodologic aspects. Scand J Gastroenterol 24, 351-358.

17. Arslan G, Atasever T, Cindoruk M et al. (2001) (51)CrEDTA colonic permeability and therapy response in patients with ulcerative colitis. Nucl Med Commun 22, 997-1001.

18. Bovee-Oudenhoven IM, ten Bruggencate SJ, Lettink-Wissink ML et al. (2003) Dietary fructooligosaccharides and lactulose inhibit intestinal colonisation but stimulate translocation of salmonella in rats. Gut 52,1572-1578.

19. Reeves PG, Nielsen FH \& Fahey GC, Jr. (1993) AIN-93 purified diets for laboratory rodents: final report of the American Institute of Nutrition ad hoc writing committee on the reformulation of the AIN-76A rodent diet. J Nutr 123, 1939-1951. 
20. Binnerts W, Van het Klooster A \& Frens A (1968) Soluble chromium indicator measured by atomic absorption in digestion experiments. Vet Rec 82, 470.

21. Sesink AL, Termont DS, Kleibeuker JH et al. (1999) Red meat and colon cancer: the cytotoxic and hyperproliferative effects of dietary heme. Cancer Res 59, 5704-5709.

22. Bovee-Oudenhoven I, Termont D, Dekker R et al. (1996) Calcium in milk and fermentation by yoghurt bacteria increase the resistance of rats to Salmonella infection. Gut 38, 59-65.

23. Lapre JA, Termont DS, Groen AK et al. (1992) Lytic effects of mixed micelles of fatty acids and bile acids. Am J Physiol 263, G333-337.

24. Bovee-Oudenhoven IM, Termont DS, Heidt PJ et al. (1997) Increasing the intestinal resistance of rats to the invasive pathogen Salmonella enteritidis: additive effects of dietary lactulose and calcium. Gut 40, 497-504.

25. Cummings JH (1981) Short chain fatty acids in the human colon. Gut 22, 763-779.

26. Hashizume K, Tsukahara T, Yamada K et al. (2003) Megasphaera elsdenii JCM1772T normalizes hyperlactate production in the large intestine of fructooligosaccharide-fed rats by stimulating butyrate production. J Nutr 133, 3187-3190.

27. Ten Bruggencate SJ, Bovee-Oudenhoven IM, Lettink-Wissink ML et al. (2005) Dietary fructooligosaccharides increase intestinal permeability in rats. J Nutr 135, 837-842.

28. Lim TS, Vedula SR, Hui S et al. (2008) Probing effects of $\mathrm{pH}$ change on dynamic response of Claudin-2 mediated adhesion using single molecule force spectroscopy. Exp Cell Res 314, 2643-2651.

29. Menconi MJ, Salzman AL, Unno N et al. (1997) Acidosis induces hyperpermeability in Caco-2BBe cultured intestinal epithelial monolayers. Am J Physiol 272, G1007-1021.

30. Unno N, Menconi MJ, Smith M et al. (1997) Hyperpermeability of intestinal epithelial monolayers is induced by NO: effect of low extracellular pH. Am J Physiol 272, G923-934.

31. Mangell P, Nejdfors P, Wang M et al. (2002) Lactobacillus plantarum 299v inhibits Escherichia coliinduced intestinal permeability. Dig Dis Sci 47, 511-516.

32. Van der Meer R, Termont DS \& De Vries HT (1991) Differential effects of calcium ions and calcium phosphate on cytotoxicity of bile acids. Am J Physiol 260, G142-147.

33. Saunders DR, Hedges JR, Sillery J et al. (1975) Morphological and functional effects of bile salts on rat colon. Gastroenterology 68, 1236-1245.

34. Bovee-Oudenhoven IM, Wissink ML, Wouters JT et al. (1999) Dietary calcium phosphate stimulates intestinal lactobacilli and decreases the severity of a salmonella infection in rats. J Nutr 129, 607-612.

35. Ten Bruggencate SJ, Bovee-Oudenhoven IM, Lettink-Wissink ML et al. (2003) Dietary fructooligosaccharides dose-dependently increase translocation of salmonella in rats. J Nutr 133, 2313-2318.

36. Alaimo K, McDowell MA, Briefel RR et al. (1994) Dietary intake of vitamins, minerals, and fiber of persons ages 2 months and over in the United States: Third National Health and Nutrition Examination Survey, Phase 1, 1988-91. Adv Data, 1-28. 
General discussion 
Inflammatory bowel disease (IBD) affects millions of people all over the world ${ }^{(1)}$. Still, its etiology and pathogenesis are only beginning to be unveiled. The most widely held hypothesis on the pathogenesis of IBD is that the inflammatory process in the intestine results from mucosal barrier dysfunction, microbial factors and dysregulated immune system reactivity in a genetically susceptible host ${ }^{(2-3)}$. This highly complicated process of immune response and chronic intestinal inflammatory activity is also of importance in the broader concept of gut health. Ultimately, optimal regulation of the various determinants involved is required to achieve a healthy gut. Since environmental factors, such as diet, clearly can modulate these determinants ${ }^{(2,4)}$, the nutritional modulation of intestinal inflammation was studied in this thesis.

\section{THE COLON AND ITS INVOLVEMENT IN GUT HEALTH}

The findings of the research described in this thesis will be discussed from the perspective how colonic function is involved in gut health and hence the diverse possibilities to intervene in these processes, as depicted in Figure 1. The different possible intervention targets, indicated by numbers in Figure 1, will be addressed one by one for their ability to be modulated by the nutrients that were the subject of investigation in this thesis.

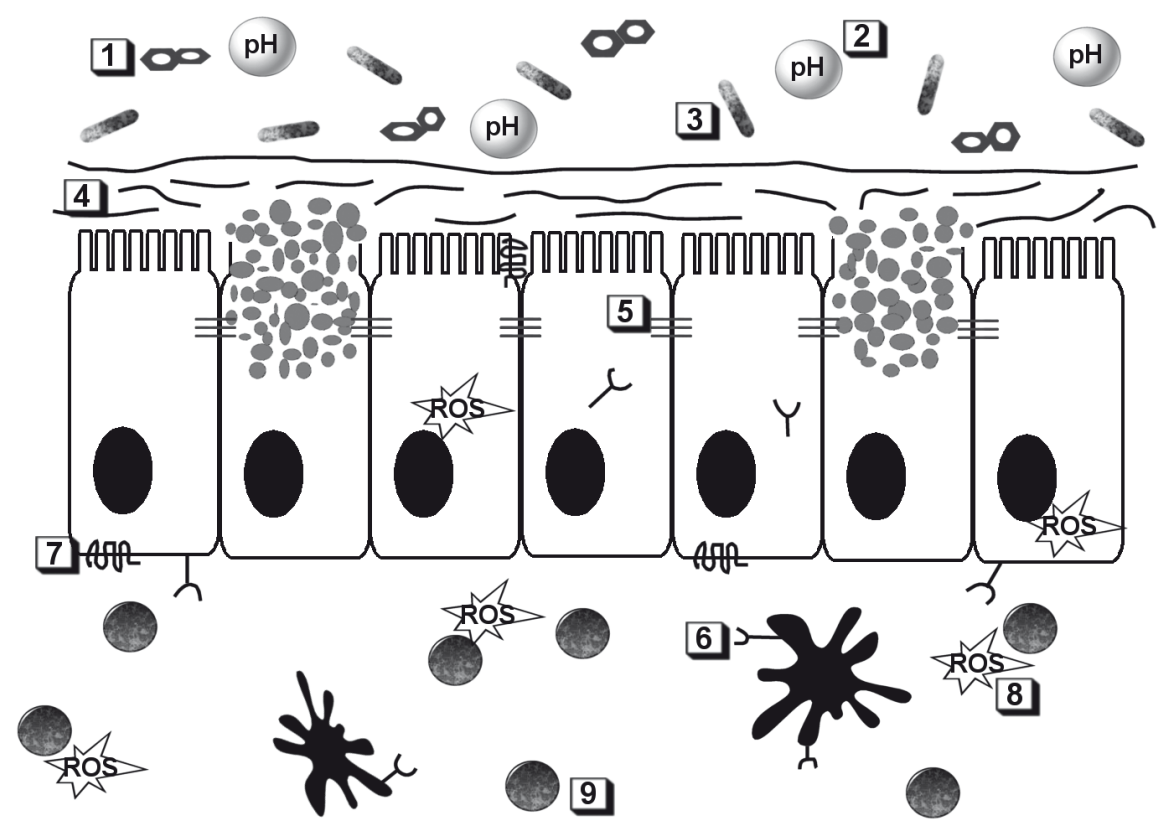

Figure 1: Schematic picture of the colonic epithelial barrier. Each number depicted in this figure represents a possibility to modulate or intervene in the colonic barrier: 1, irritants in the colonic lumen; 2 , buffering capacity in the colonic lumen; 3, microbiota; 4, mucus layer; 5, tight junctions; 6, toll-like receptors; 7, calcium-sensing receptor; 8 , oxidative stress; 9 , immune cells. 


\section{Irritants in the colonic lumen}

Modulation of the luminal contents of the colon (not including the gut microbiota) is generally not considered in the pharmacological approach to IBD therapy, although many opportunities exist here. Firstly, bile acids and fatty acids that escape absorption in the small intestine can be highly irritating to the colonic epithelium ${ }^{(5-6)}$. As shown earlier, dietary calcium can precipitate these irritating surfactants, resulting in a reduced cytotoxicity of the fecal stream, which supports mucosal integrity ${ }^{(7-8)}$. It has also been shown in this thesis that luminal cytotoxicity was lower on a calcium diet in HLA-B27 transgenic rats (Chapter 3) and healthy rats (Chapter 6). This effect of dietary calcium on luminal cytotoxicity in the colon may possibly explain the beneficial influence of calcium on intestinal permeability, which is one of the important findings in this thesis. Nevertheless, the Ussing chamber results (Chapter 5) do not support this hypothesis, as fecal water derived from calcium-fed rats was not different from fecal water of control rats in influencing colonic permeability in these ex-vivo experiments. However, the exposure time of the colonic epithelium to the fecal water in the Ussing chambers is not comparable with that of the in vivo situation. Furthermore, the lower luminal cytotoxicity due to calcium might also indirectly influence mucosal integrity, and in particular intestinal permeability, via changes in the gut microbiota, which is not represented in the Ussing chamber. For example, the shift to a less cytotoxic fecal stream by calcium favors the growth of endogenous lactobacilli ${ }^{(9)}$. Opposite to calcium, dietary heme-enhanced cytotoxicity of the luminal contents has been shown to cause damage to colonic epithelial cells. Involvement of a heme-induced irritating metabolite is suggested in this process ${ }^{(10)}$. Dietary heme also increased luminal cytotoxicity in the experiments described in this thesis, which was associated with adverse effects on colitis development (Chapter 2). This thesis shows, at least in rats, that luminal irritants and related cytotoxicity might be an important factor in gut health, since lowering of cytotoxicity is associated with beneficial effect on colitis development, while increasing cytotoxicity is accompanied by adverse effects on colonic inflammation. Since it has been shown that calcium can counteract heme-induced intestinal stress ${ }^{(11)}$, it would be interesting to study the influence of dietary heme with or without supplemental calcium on the severity of colitis development in HLA-B27 transgenic rats. In a broader perspective, manipulating the concentration of irritants in the intestinal lumen might be an interesting approach in both nutritional as well as pharmacological research. It is surprising that so little research is aimed at influencing the intestinal contents themselves (not including the gut microbiota), while the gut with its enormous surface area is continuously exposed to it.

\section{Buffering capacity in the colonic lumen}

Another factor related to the colonic lumen, and sensitive to nutritional modulation, is the buffering capacity of the contents. Just like an increased luminal cytotoxicity, a low $\mathrm{pH}$, for example caused by fermentation of non-digestible carbohydrates into organic acids, might damage the delicate colonic epithelium. In this way, dietary calcium might be of importance, since solubilization of calcium phosphate in the colon can increase the buffering capacity and thereby limit excessive acidification ${ }^{(12)}$. Interestingly, in this 
thesis, an association was observed between buffering capacity and its influence on intestinal permeability (Chapter 6). Therefore, modulation of the luminal buffering capacity might be another possibility to intervene in the colonic lumen.

\section{Microbiota}

Extensive and still growing research interest is focused on the modulation of the intestinal microbiome to improve gut health. There is abundant evidence that commensal bacteria are involved in the pathogenesis of IBD ${ }^{(3,13-15)}$. Effects of dietary calcium on the intestinal microbiota are described in this thesis (Chapter 5), and elsewhere ${ }^{(9,16)}$. Since supplemental calcium ameliorated colitis development (Chapters 3 and 4), it can be speculated that the microbiota is involved in these calcium-induced effects on intestinal inflammation. We hypothesize that the beneficial influence of calcium on luminal cytotoxicity affects the gut microbiome. Furthermore, a direct effect of calcium on the microbiota or its metabolites might be possible. Lipopolysaccharide (LPS) is the major component of the outer membranes of Gram-negative bacteria and is associated with pathogenic and inflammatory capability. Since the structure of LPS shows physical similarities to that of bile acids and fatty acids, calcium might precipitate these molecules as well. Binding of LPS to calcium might prevent leakage of this component through the colonic mucosa. Indeed, the increase in anti-LPS antibodies during colitis development was less in HLA-B27 transgenic rats fed the calcium diet compared to HLA-B27 rats fed the control diet (Chapter 3). Moreover, preliminary experiments show that dietary calcium induces a shift in the predominant species of the colonic bacterial community from Gram-negative Bacteroidetes to Gram-positive Firmicutes, which was determined by quantitative PCR on feces (data not shown). This effect might be explained by binding of LPS-containing Gram-negative species to amorphous calcium phosphate, or the lower luminal cytotoxity due to calcium, which is particularly stimulating for sensitive Gram-positive lactobacilli ${ }^{(9)}$. Further research in this direction by using pyrosequencing techniques might be worthwhile. Furthermore, dietary heme was demonstrated to affect the colonic bacteria (Chapter 2) in the opposite direction compared to supplemental calcium. The effect of heme on the microbiome was associated with adverse effects on colitis development, which makes it even more interesting to study the combination of calcium and heme in the HLA-B27 rat model as mentioned earlier. Research on modulation of the microbiota aimed at improving gut health has gained attention, especially in the field of prebiotics and probiotics. However 'ordinary' or habitual dietary components should not be ignored for their ability to modify the gut microbiome.

\section{Mucus layer}

Separating and protecting the intestinal epithelial cells from exposure to potentially irritating luminal contents is accomplished by the mucus layer. Modulating the properties of this layer yields an interesting opportunity in IBD research, in particular with regard to the viscosity or density of the mucus layer, which might influence microbial adhesion and infiltration. Correspondingly, it has been shown that none of the biopsies from patients with colonic inflammation showed an intact mucus barrier ${ }^{(17)}$. We speculate 
that the beneficial effect of dietary calcium on intestinal permeability (Chapters 5 and 6) and colitis development in general (Chapters 3 and 4) involves the quality of the mucus layer. Literature indicates that calcium may be important for the structure of the mucus gel by providing cross-links between the proteins, which subsequently influences the viscosity and/or density ${ }^{(18-20)}$. Investigation of the biochemical and physical properties of the mucus layer is challenging because of its structural complexity, which is difficult to mimic in vitro ${ }^{(21)}$. Preliminary studies on the effect of calcium on mucus viscosity performed in our lab show inconsistent results. This might be due to the fact that mucins become smaller and more dense when calcium is added. It is suggested that calcium binds to the negative charges on the mucins, subsequently causing aggregation and contraction of the mucin macromolecules. This causes a decrease of the viscosity of the total suspension containing the mucins ${ }^{(20,22)}$, while the intrinsic viscosity of the macromolecular mucin complex itself might be increased. A possibility to overcome these in vitro difficulties of mucus layer studies is the use of confocal scanning laser microscopy (CSLM). A fluorescent dye can be applied at the luminal site of a fresh piece of colonic tissue, which still has an intact mucus layer. Before application of the dye, this tissue section has been treated with or without calcium, after which permeation of the dye can be monitored by CSLM. Another way to study the mucus layer is the fixation of a piece of colonic tissue in Carnoy's solution, which preserves the mucus gel ${ }^{(23)}$. After feeding rats either a control diet or a calcium diet, possible differences in the mucus layer and potential permeation of luminal bacteria can be histologically visualized by this fixation technique ${ }^{(24)}$. So, some interesting approaches are available to investigate the mucus layer, in particular for mechanistic research purposes.

\section{Tight junctions}

The environment of the epithelial tight junction is the principal determinant of mucosal paracellular permeability ${ }^{(25-26)}$. As already mentioned above, the beneficial effect of dietary calcium on intestinal permeability is an important finding of this thesis. Supplemental calcium not only decreases intestinal permeability in colitis models (Chapters 3 and 4), but also in healthy rats (Chapters 5 and 6). It would be interesting to study the underlying mechanism at the level of the tight junction, such as altered production or relocalization of certain tight junction proteins, by using immunofluorescence on histological sections. More important, however, is to demonstrate and validate the effect of calcium in humans. Patients having an increased intestinal permeability, as in IBD but also in untreated celiac disease and post-infectious irritable bowel syndrome, could consume either placebo or calcium supplements, while their intestinal permeability is determined non-invasively by using non-digestible probes like certain disaccharides, poly-ethylene glycol (PEG) molecules or (nonradioactive) CrEDTA. Intestinal permeability is expected to decrease in the calcium supplemented patients and clinical relevance would greatly increase if this could be linked to amelioration of disease symptoms. A calcium intervention in healthy subjects might be another possibility. It is still unclear whether intestinal permeability can be decreased in healthy subjects, and whether this will protect against a subsequent stressor. After some weeks on either a low or a high calcium diet, subjects can be 
challenged by a colonic stressor, such as bile acids, synthetic permeation enhancers, rectal enemas or delayed-release capsules which target the colon and contain for example NSAIDS. In the meantime, intestinal permeability is measured using one of the above mentioned probes. We would hypothesize that the healthy subjects on the high calcium diet obtain a lower permeability and are subsequently better protected against this colonic stressor. Thus, the protective effect of supplemental calcium on intestinal permeability is definitely worthwhile to continue investigating.

\section{Toll-like receptors}

This topic is strongly related to the importance of the microbiota in gut health (paragraph 3). Toll-like receptors (TLRs) are involved in microbial sensing, which subsequently leads to immune system activation and response ${ }^{(13,27-28)}$. Targeting TLRs in order to prevent or treat IBD is an emerging field of research. Several compounds, with either agonistic or antagonistic activity to TLRs, are the focus of current pharmacological and probiotic research ${ }^{(29)}$. To our knowledge, nutrients that target TLRs have not been identified yet. On the other hand, pivotal mechanisms underlying the dietary effects on gut health can be unraveled by using pharmaceutical TLR agonists and/or antagonists. In particular, the mechanism behind the beneficial effect of calcium on colitis development could be investigated using TLR-targeting drugs. For example, administration of a TLR4 antagonist to HLA-B27 rats, on either a low or high calcium diet, might give insight into the proposed mechanism that calcium influences gut health by modulating the microbiome. According to this hypothesis, a calcium diet will not be effective in the experiment with a TLR4 antagonist. In conclusion, a nutritional approach to directly influence TLRs might not be possible; however, the current interest in TLRs opens new ways to investigate mechanistic properties of nutrients.

\section{Calcium-sensing receptor}

The calcium-sensing receptor is another receptor in the colon, suggested to be responsive to nutritional or pharmaceutical interventions. It is known that this receptor is expressed on epithelial cells along the gastro-intestinal tract, even on the apical site of these cells ${ }^{(30-31)}$. This is remarkable, since the luminal calcium concentration is subject to large variations as it is largely dependent on the meal consumed. It seems unrealistic to think that these large variations in calcium concentration can be appropriately transmitted by the apical calcium-sensing receptor. However, it is tempting to speculate that activation of the calcium-sensing receptor by dietary calcium reduces proliferation and induces differentiation of the colonic epithelium ${ }^{(32)}$. Furthermore, stimulation of this receptor is also involved in anti-diarrheal effects ${ }^{(33)}$, which makes it even more reasonable for it to be a target for luminal calcium in our intervention studies. Dietary calcium prevents the increase in diarrhea during colitis development as described in this thesis (Chapters 3 and 4). To the best of our knowledge, no evidence exists from in vivo experiments demonstrating that specific activation of the calcium-sensing receptor has these beneficial effects on colon health as is proposed for luminal calcium. These studies have still to be done. Since the functioning of the receptor in vitro and ex vivo, e.g. in cell lines or Ussing chambers respectively, is said to decline very fast, and 
calcium-sensing receptor knock-out mice are not viable, solid research on this receptor is challenging. A possibility is the intestine-specific knock-down of the receptor by for example small interfering RNA. Thus, it is certainly worthwhile to explore the calciumsensing receptor and its possible mechanistic involvement in the protective effects of calcium in the colon.

\section{Oxidative stress}

Oxidative stress is suggested to play an important role in the pathogenesis of $\operatorname{IBD}^{(34)}$, and it offers several opportunities to intervene. In the research performed in this thesis, particular interest was paid to interventions with dietary antioxidants as scavengers of reactive oxygen species (ROS), the compounds which play a central role in oxidative stress. So far, our interventions, i.e. calcium and heme, were active in the lumen of the colon, while antioxidant supplementation is supposed to function at the mucosal level (Chapter 4). However, supplemental antioxidants in the HLA-B27 model did not protect against colitis development, although the results are difficult to interpret since the respiratory burst was compromised in these rats (Chapter 4). Nevertheless, the lack of any effect of antioxidants on colitis severity was further demonstrated in other animal experiments, performed for this thesis (data not shown). These antioxidants have been investigated in the trinitrobenzene sulfonic acid (TNBS) experimental colitis model and no beneficial effects were observed on inflammatory markers such as myeloperoxidase. Hardly any convincing evidence exists in the literature in which oxidative damage or increased ROS production is associated with decreased antioxidant capacity or increased intestinal inflammatory activity. Moreover, it is even questionable whether the intestinal antioxidant capacity is deficient in intestinal disease, and whether it is possible to increase this capacity in a healthy person. Although antioxidants have gained a lot of attention in previous years, hardly any intervention study with IBD patients using antioxidants can be found in the literature. This raises questions whether these studies simply have not been performed, or whether there is publication bias, i.e. disappointing results have not been published. Sound pre-clinical scientific evidence should be provided before starting clinical trials studying efficacy of antioxidant supplementation. Importantly, a meta-analysis even showed an increased risk of allcause mortality in healthy adults due to supplemental antioxidants, emphasizing that supplementation with antioxidants has to be done with caution ${ }^{(35)}$. Another difficulty in antioxidant supplementation is what antioxidant to select among the many different antioxidants. In this thesis, a mixture of antioxidants was chosen as supplementation, with each antioxidant having its own particular characteristics (Chapter 4). Subsequently, an appropriate dose of the antioxidant should be established ${ }^{(36)}$. As the choice of antioxidants is so wide, often hardly any dosing experiments can be found concerning a particular antioxidant of interest in the literature, which makes it even more challenging to perform proper experiments. Moreover, some antioxidants can even become pro-oxidant when combined with other nutrients, for instance the combination of vitamin $\mathrm{C}$ and iron. So, in the area of antioxidant research, many relevant questions still remain unanswered and the focus should be on antioxidative networking rather than individual antioxidants ${ }^{(37-38)}$. Although already quite a number of food products in 
the supermarket contain supplemental antioxidants, experimental evidence about their functionality is often lacking.

\section{Immune cells}

Finally, the immune cells, such as dendritic cells, macrophages, neutrophils and T cells, which execute the actual inflammatory response can be the target of an intervention in the colon. Specifically targeting receptors or signaling molecules of these cells, such as cytokines, seems more likely for biological therapy. The use of biological therapy in IBD is increasingly recognized as a useful approach to treat patients. Many biological agents are either already approved or are likely to enter the market, such as infliximab and natalizumab ${ }^{(39)}$. To directly influence these targets, a compound should have perfect specific binding properties to a particular receptor or signaling molecule. For example, infliximab is an antibody against the cytokine tumor necrosis factor and natalizumab binds specifically to the adhesion molecule $\alpha 4$ integrin. Therefore, the outcomes of the research described in this thesis regarding immune responses are probably secondary consequences of earlier impact in the nutrient-gut effect cascade due to a nutritional intervention. Since for example dietary calcium exerts its protective influence in the colonic lumen, subsequent steps of the inflammatory cascade in the mucosa will also be beneficially affected. So, the nutritional effects on the immune response in the experiments described in this thesis are most likely indirect influences.

\section{NUTRITIONAL INTERVENTIONS}

When investigating nutritional modulation of intestinal inflammation, some (methodological) considerations have to be taken into account. To start with, when performing nutritional experiments, effects are often, if not always, much smaller than in the case of pharmacological interventions. However, and importantly, nutritional interventions benefit from having hardly any unwanted adverse effects and are hence not time-limited, which makes them therapeutically attractive.

It is considered unethical to test a nutrient in a human trial without prior evidence of potential efficacy. For example, supplemental calcium has barely been implicated to play a role in the amelioration of colitis in the literature and should therefore first be tested in animal models mimicking IBD. Furthermore, the colon can be easily investigated in animals by simply taking it out at the end of the experiment, while in humans only mucosal specimens can be obtained by performing endoscopic investigation, which implies substantial discomfort to the subject and often prior cleansing of the colon, which disturbs the natural environment. Most initial experiments are short-term, as this is practically more feasible in animal studies. However, dietary effects or disease processes may take much longer to bring about any change. Nevertheless, animal studies allow the opportunity for more flexibility in design and outcome measures, and in the ability to control experimental and confounding variables than can be achieved in human studies. For example, animals are fed a standardized diet, being the same every day, which is obviously not the case for humans. This is particularly important 
to bear in mind when considering possible interactions of the nutrient of interest with other nutrients or the food matrix in general. An evident example is the adverse effect of short-chain fructo-oligosaccharides (scFOS) on gut health, which is almost absent if calcium is also added to the $\operatorname{diet}^{(40)}$. Likewise, chlorophyll is able to prevent the hemeinduced cytotoxic effects in the colon ${ }^{(41)}$.

An important methodological consideration that holds for all animal studies is the extrapolation to the human situation. Three aspects should be considered in this respect, namely the nutritional relevance, the degree of similarity with regard to the gastrointestinal tract and to what extent the animal model of chronic intestinal inflammation resembles human disease. Nutritional relevance should be a central issue when a nutritional intervention study is being designed. The extrapolation of the concentration of the nutrient in the animal diet to that in the human situation is discussed in the various manuscripts of this thesis (Chapters 2 through 6). In nutritional studies one commonly uses a rather high but not unrealistic concentration of the nutrient of interest in order to show proof of concept. For example, the heme concentration used in this thesis (Chapter 2) corresponds to a beef intake of $500 \mathrm{~g} /$ day in humans ${ }^{(41)}$. In subsequent studies, a dose-response effect can be studied by using lower concentrations of the nutrient of interest. In the case of antioxidant supplementation, it is common to take into account the intake of antioxidant supplements in the habitual diet when extrapolating from the animal diet to human dietary intake.

Secondly, it is important to consider the differences in gastro-intestinal physiology between rats and humans. Rats have a large cecum, while humans have a poorly defined cecal region, which is continuous with the colon ${ }^{(42)}$. This is of particular importance if the microbiota is expected to be involved in the dietary effects of the nutrient of interest. However, the number and general composition of bacteria in the rat cecum and colon, and the human colon are largely comparable ${ }^{(42)}$. Therefore, in case of e.g. scFOS supplementation (Chapters 5 and 6), the combined physiological effects of scFOS fermentation in the cecum and colon of rats will likely occur in the colon in humans. Previous work from our lab showed that the effect of scFOS is also clearly present in the colon of rats. For example, the lactic acid concentration increased in feces of rats fed scFOS compared to control rats ${ }^{(43)}$. In addition, dietary scFOS have similar effects in the cecum and colon of rats with regard to mucosal inflammation ${ }^{(43)}$, mucin concentration and mucosal lactobacilli and enterobacteria ${ }^{(44)}$. Similar considerations about the extent of similarity of the microbiota have to be taken into account for a nutritional intervention with calcium, since it is hypothesized that the protective effect of calcium is mediated by the gut microbiome or its metabolites. As both rats and humans have comparable bacterial species in the lower intestine, the calcium-induced modulation of the microbiota should still be present in humans ${ }^{(42)}$, but final proof needs to come from a dietary intervention study in humans.

Finally, the degree of resemblance of the rat IBD model to human disease should be considered. As mentioned in the general introduction (Chapter 1), several animal models mimicking human IBD exist, all with their own characteristics ${ }^{(45-48)}$. The TNBS colitis model used in this thesis (Chapter 2) shares many features with human Crohn's disease $^{(49)}$. The HLA-B2 7 rat model, also applied in this thesis (Chapters 3 and 4), is very 
useful for studying the effect of the microbiota on intestinal inflammation ${ }^{(46)}$, since HLAB27-expressing innate immune cells and the presence of enteric bacteria are essential in the development of colitis in this model ${ }^{(50)}$. There is no animal model sharing all characteristics of human IBD. This is not surprising since disease pathogenesis in IBD patients is also very heterogeneous. This heterogeneity also implies that the potential influence of an (nutritional) intervention can be different between various patients (responders and non-responders), in particular if a genetic predisposition is involved. Consequently, in view of the animal models, it is often requested by the scientific community to demonstrate efficacy of a nutrient in several different experimental colitis models to substantiate the proof and increase the likelihood of success in human follow-up studies. The latter is obviously the ultimate goal.

\section{FINAL CONCLUSIONS AND OVERALL REFLECTIONS}

In this thesis, the nutritional modulation of intestinal inflammation was studied. This is shown to be possible in animal models mimicking IBD. Moreover, some light is shed on the (patho)physiologic mechanisms involved. In this respect the following conclusions can be drawn:

1. Dietary heme, mimicking red meat, adversely affects colitis in rats. It is speculated that the heme-induced luminal cytotoxicity impairs intestinal mucosal recovery, subsequently aggravating colitis severity.

2. Dietary calcium ameliorates colitis development in HLA-B27 transgenic rats. A decrease in intestinal permeability is suggested to play an important role, and particularly colonic permeability is beneficially affected by calcium. The buffering capacity in the colonic lumen is associated with the beneficial effect of calcium on permeability. Furthermore, it is hypothesized that the intestinal microbiota and/or the mucus layer is involved in the protective effect of calcium (Figure 2).

3. The HLA-B27 transgenic rat model is not an appropriate model to study the effect of supplemental antioxidants. Therefore, a definitive conclusion cannot be drawn about the potential effects of antioxidant supplementation on intestinal inflammation.

Hence, nutritional modulation of intestinal inflammation is possible, at least in rats, as is shown in this thesis for calcium and heme. Most importantly, it is shown for the first time that dietary calcium in rats, besides being protective against intestinal infection, also is able to attenuate chronic intestinal inflammation, as in IBD. This shows the usefulness of an easily applicable nutritional intervention in the modulation of intestinal 


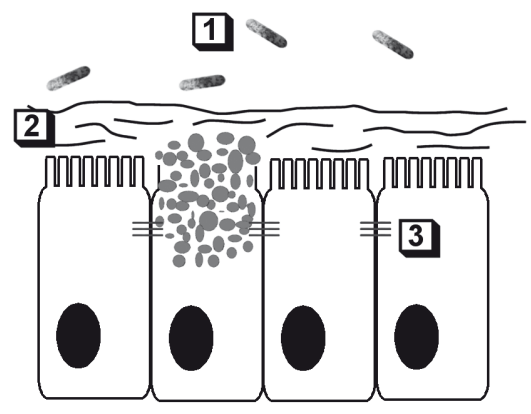

Figure 2: Schematic picture of the colonic epithelial barrier, reflecting the research performed in this thesis. The most interesting options for further research through which dietary calcium influences intestinal inflammation, and in a broader sense gut health, are depicted by the numbers in this figure: 1, microbiota; 2, mucus layer; 3 , tight junctions/paracellular permeability.

inflammation, which is attractive for the food industry interested in development of functional foods. Moreover, it contributes to the dossier that is built to substantiate a new claim on calcium and gut health. Interestingly, the beneficial effects of dietary calcium are not restricted to inflammation in IBD, as calcium is also protective against intestinal infections and colon cancer. This suggests that calcium in the gut affects some generic mechanisms. This should be validated in the human situation.

Research on nutritional modulation of IBD is still in its infancy, in animals, but even more so in humans. The analysis of nutritional interventions is challenging because the expected effects are rather small. Hence, nutrition cannot replace pharmacological interventions in the case of IBD. Pharmaceutical compounds will (always) remain the first choice of therapy. Nevertheless, nutrients can be used as a supportive add-on strategy. In particular supplemental calcium is interesting, since it has been shown in this thesis that it beneficially influences intestinal permeability and probably favorably affects the microbiota. These targets are not of special interest for the pharmaceutical industry nowadays and might therefore be an additive approach to existing therapy for IBD patients. In a broader perspective, the same applies for other diseases affecting gut health, such as intestinal infection. There are clear and realistic opportunities for beneficial nutritional interventions, but further research is necessary. The lack of adverse effects of nutritional 'therapy' makes it an favorable choice to improve gut health. Furthermore, patients with intestinal disorders are generally well-motivated to adjust their diet, since it provides them with a 'natural' approach to control their disease.

\section{REFERENCES}

1. Hanauer SB (2006) Inflammatory bowel disease: epidemiology, pathogenesis, and therapeutic opportunities. Inflamm Bowel Dis 12 Suppl 1, S3-9.

2. Sartor RB (2006) Mechanisms of disease: pathogenesis of Crohn's disease and ulcerative colitis. Nat Clin 
Pract Gastroenterol Hepatol 3, 390-407.

3. Xavier RJ \& Podolsky DK (2007) Unravelling the pathogenesis of inflammatory bowel disease. Nature 448, 427-434.

4. Danese S, Sans M \& Fiocchi C (2004) Inflammatory bowel disease: the role of environmental factors. Autoimmun Rev 3, 394-400.

5. Hofmann AF (1999) Bile Acids: The Good, the Bad, and the Ugly. News Physiol Sci 14, 24-29.

6. Saunders DR, Hedges JR, Sillery J et al. (1975) Morphological and functional effects of bile salts on rat colon. Gastroenterology 68, 1236-1245.

7. Govers MJ, Termont DS, Lapre JA et al. (1996) Calcium in milk products precipitates intestinal fatty acids and secondary bile acids and thus inhibits colonic cytotoxicity in humans. Cancer Res 56, 3270-3275.

8. Lapre JA, De Vries HT, Koeman JH et al. (1993) The antiproliferative effect of dietary calcium on colonic epithelium is mediated by luminal surfactants and dependent on the type of dietary fat. Cancer Res 53, 784-789.

9. Bovee-Oudenhoven IM, Wissink ML, Wouters JT et al. (1999) Dietary calcium phosphate stimulates intestinal lactobacilli and decreases the severity of a salmonella infection in rats. J Nutr 129, 607-612.

10. Sesink AL, Termont DS, Kleibeuker JH et al. (1999) Red meat and colon cancer: the cytotoxic and hyperproliferative effects of dietary heme. Cancer Res 59, 5704-5709.

11. Sesink AL, Termont DS, Kleibeuker JH et al. (2001) Red meat and colon cancer: dietary haem-induced colonic cytotoxicity and epithelial hyperproliferation are inhibited by calcium. Carcinogenesis 22, 16531659.

12. Remesy C, Levrat MA, Gamet L et al. (1993) Cecal fermentations in rats fed oligosaccharides (inulin) are modulated by dietary calcium level. Am J Physiol 264, G855-862.

13. Abraham C \& Cho JH (2009) Inflammatory bowel disease. N Engl J Med 361, 2066-2078.

14. Round JL \& Mazmanian SK (2009) The gut microbiota shapes intestinal immune responses during health and disease. Nat Rev Immunol 9, 313-323.

15. Sartor RB (2008) Microbial influences in inflammatory bowel diseases. Gastroenterology 134, 577-594.

16. Bovee-Oudenhoven IM, Termont DS, Weerkamp AH et al. (1997) Dietary calcium inhibits the intestinal colonization and translocation of Salmonella in rats. Gastroenterology 113, 550-557.

17. Swidsinski A, Loening-Baucke V, Theissig F et al. (2007) Comparative study of the intestinal mucus barrier in normal and inflamed colon. Gut 56, 343-350.

18. Crowther RS, Marriott C \& James SL (1984) Cation induced changes in the rheological properties of purified mucus glycoprotein gels. Biorheology 21, 253-263.

19. Raynal BD, Hardingham TE, Sheehan JK et al. (2003) Calcium-dependent protein interactions in MUC5B provide reversible cross-links in salivary mucus. J Biol Chem 278, 28703-28710.

20. Forstner JF \& Forstner GG (1976) Effects of calcium on intestinal mucin: implications for cystic fibrosis. Pediatr Res 10, 609-613.

21. Perez-Vilar J \& Mabolo R (2007) Gel-forming mucins. Notions from in vitro studies. Histol Histopathol $22,455-464$.

22. Forstner JF \& Forstner GG (1975) Calcium binding to intestinal goblet cell mucin. Biochim Biophys Acta 386, 283-292.

23. Matsuo K, Ota H, Akamatsu T et al. (1997) Histochemistry of the surface mucous gel layer of the human colon. Gut 40, 782-789.

24. Johansson ME, Phillipson M, Petersson J et al. (2008) The inner of the two Muc2 mucin-dependent mucus layers in colon is devoid of bacteria. Proc Natl Acad Sci U S A 105, 15064-15069.

25. Clayburgh DR, Shen L \& Turner JR (2004) A porous defense: the leaky epithelial barrier in intestinal disease. Lab Invest 84, 282-291.

26. Turner JR (2009) Intestinal mucosal barrier function in health and disease. Nat Rev Immunol 9, 799809.

27. Karin M, Lawrence $T$ \& Nizet V (2006) Innate immunity gone awry: linking microbial infections to chronic inflammation and cancer. Cell 124, 823-835.

28. Macdonald TT \& Monteleone G (2005) Immunity, inflammation, and allergy in the gut. Science 307, 1920-1925.

29. Hennessy EJ, Parker AE \& O’Neill LA (2010) Targeting Toll-like receptors: emerging therapeutics? Nat Rev Drug Discov 9, 293-307. 
30. Hebert SC, Cheng S \& Geibel J (2004) Functions and roles of the extracellular Ca2+-sensing receptor in the gastrointestinal tract. Cell Calcium 35, 239-247.

31. Chattopadhyay N, Cheng I, Rogers K et al. (1998) Identification and localization of extracellular Ca(2+)sensing receptor in rat intestine. Am J Physiol 274, G122-130.

32. Geibel JP \& Hebert SC (2009) The functions and roles of the extracellular Ca2+-sensing receptor along the gastrointestinal tract. Annu Rev Physiol 71, 205-217.

33. Geibel J, Sritharan K, Geibel R et al. (2006) Calcium-sensing receptor abrogates secretagogue- induced increases in intestinal net fluid secretion by enhancing cyclic nucleotide destruction. Proc Natl Acad Sci U S A 103, 9390-9397.

34. Kruidenier L \& Verspaget HW (2002) Review article: oxidative stress as a pathogenic factor in inflammatory bowel disease--radicals or ridiculous? Aliment Pharmacol Ther 16, 1997-2015.

35. Bjelakovic G, Nikolova D, Gluud LL et al. (2007) Mortality in randomized trials of antioxidant supplements for primary and secondary prevention: systematic review and meta-analysis. Jama 297, 842-857.

36. Kwon KH, Murakami A, Tanaka T et al. (2005) Dietary rutin, but not its aglycone quercetin, ameliorates dextran sulfate sodium-induced experimental colitis in mice: attenuation of pro-inflammatory gene expression. Biochem Pharmacol 69, 395-406.

37. Blomhoff R, Carlsen MH, Andersen LF et al. (2006) Health benefits of nuts: potential role of antioxidants. Br J Nutr 96 Suppl 2, S52-60.

38. van Haaften RI, Haenen GR, Evelo CT et al. (2003) Effect of vitamin E on glutathione-dependent enzymes. Drug Metab Rev 35, 215-253.

39. Rutgeerts P, Vermeire S \& Van Assche G (2009) Biological therapies for inflammatory bowel diseases. Gastroenterology 136, 1182-1197.

40. Ten Bruggencate SJ, Bovee-Oudenhoven IM, Lettink-Wissink ML et al. (2004) Dietary fructooligosaccharides and inulin decrease resistance of rats to salmonella: protective role of calcium. Gut 53, 530-535.

41. de Vogel J, Jonker-Termont DS, van Lieshout EM et al. (2005) Green vegetables, red meat and colon cancer: chlorophyll prevents the cytotoxic and hyperproliferative effects of haem in rat colon. Carcinogenesis 26, 387-393.

42. Kararli TT (1995) Comparison of the gastrointestinal anatomy, physiology, and biochemistry of humans and commonly used laboratory animals. Biopharm Drug Dispos 16, 351-380.

43. Bovee-Oudenhoven IM, ten Bruggencate SJ, Lettink-Wissink ML et al. (2003) Dietary fructooligosaccharides and lactulose inhibit intestinal colonisation but stimulate translocation of salmonella in rats. Gut 52, 1572-1578.

44. Ten Bruggencate SJ, Bovee-Oudenhoven IM, Lettink-Wissink ML et al. (2005) Dietary fructooligosaccharides increase intestinal permeability in rats. J Nutr 135, 837-842.

45. Elson CO, Sartor RB, Tennyson GS et al. (1995) Experimental models of inflammatory bowel disease. Gastroenterology 109, 1344-1367.

46. Hibi T, Ogata H \& Sakuraba A (2002) Animal models of inflammatory bowel disease. J Gastroenterol 37, 409-417.

47. Hoffmann JC, Pawlowski NN, Kuhl AA et al. (2002) Animal models of inflammatory bowel disease: an overview. Pathobiology 70, 121-130.

48. Jurjus AR, Khoury NN \& Reimund JM (2004) Animal models of inflammatory bowel disease. J Pharmacol Toxicol Methods 50, 81-92.

49. Morris GP, Beck PL, Herridge MS et al. (1989) Hapten-induced model of chronic inflammation and ulceration in the rat colon. Gastroenterology 96, 795-803.

50. Sartor RB (2000) Colitis in HLA-B27/beta 2 microglobulin transgenic rats. Int Rev Immunol 19, 39-50. 
Chapter 7

1 Chapter 7

| 124 
Summary 
Inflammatory bowel disease (IBD) affects millions of people all over the world. It comprises primarily two disorders of chronic intestinal inflammation: ulcerative colitis and Crohn's disease. The etiology and pathogenesis of IBD are only beginning to be unveiled. The most widely held hypothesis on the pathogenesis of IBD is that the inflammatory process in the intestine results from mucosal barrier dysfunction, microbial factors and dysregulated immune system reactivity in a genetically susceptible host. This highly complicated process of immune response and chronic intestinal inflammatory activity is also of importance in the broader concept of gut health. Environmental factors, such as diet, can modulate these determinants of the pathogenesis of IBD. However, research on dietary modulation of IBD is in its infancy. Therefore, in this thesis, the nutritional modulation of intestinal inflammation was studied.

In chapter 2 of this thesis, the effect of dietary heme, mimicking red meat, on intestinal inflammation was investigated. Dietary heme is considered an intestinal stressor in colorectal cancer research. However, heme-induced colonic stress might also result in induction of potentially protective heat shock proteins. Rats were fed 'humanized' Western diets either with or without heme, after which colitis was chemically induced. The results of this study show that dietary heme adversely affected colitis development, despite heat shock protein induction. In particular, body weight gain, colon length and food intake were lower in rats fed the heme diet. It is speculated that the heme-induced luminal cytotoxicity impairs intestinal mucosal recovery, subsequently aggravating colitis severity. A diet high in red meat might be a risk factor for IBD development.

Chapter 3 describes the effect of supplemental calcium on intestinal inflammation. It has been shown previously that dietary calcium strengthens the mucosal barrier, which may also be of importance in IBD. Therefore, HLA-B27 transgenic rats, spontaneously developing colitis, were fed a Western-style diet containing either a low or high calcium content. The calcium diet significantly inhibited the increase in intestinal permeability and diarrhea with time in HLA-B27 rats developing colitis. Moreover, supplemental calcium prevented the colitis-induced increase in the expression of extracellular matrix remodeling genes. So, dietary calcium ameliorated several important symptoms and key mechanisms of colitis severity. These results show promise for supplemental calcium as effective adjunct therapy for IBD.

The aim of chapter $\mathbf{4}$ was to investigate whether supplementation of a mixture of antioxidants would ameliorate colitis development, and whether the combination of dietary antioxidants and calcium has additive protective effects. To this end, HLA-B27 rats were fed a control diet, a diet supplemented with the antioxidants glutathione, vitamin $\mathrm{C}$ and vitamin $\mathrm{E}$, or a diet supplemented with both the antioxidant mixture and calcium. The results in chapter $\mathbf{4}$ show that antioxidant supplementation was ineffective in HLA-B27 rats despite low colonic mucosal glutathione levels, because colitis development did not coincide with oxidative stress in this model. So, the HLA-B27 transgenic rat model is not an appropriate model to study the effect of supplemental antioxidants. As supplementation with both calcium and antioxidants attenuated colitis development, this protective effect is contributed to calcium only. Therefore, the significant protective effect of dietary calcium on intestinal inflammation shown before 
was confirmed by this study.

A decrease in intestinal permeability is suggested to play an important role in the protective effect of calcium on intestinal inflammation. Therefore, chapter $\mathbf{5}$ describes a more mechanistic study, in which it was investigated how and where in the gastrointestinal tract calcium exerts its influence on intestinal permeability. To study this, rats were fed a humanized control diet, or a similar diet supplemented with calcium. Also, the localization of the effect on intestinal permeability due to shortchain fructo-oligosaccharides (scFOS) supplementation was investigated in this study. Lactulose plus mannitol and CrEDTA were added to the diets to measure small and total intestinal permeability respectively. This study shows that both calcium and scFOS affected permeability only in the colon: calcium decreased, while scFOS increased colonic permeability. As fecal water of the dietary intervention study did not influence permeability in (ex-vivo) Ussing chambers, it is suggested that modulation of mucins and/or microbiota may be important for the in vivo effects of dietary calcium and scFOS. Chapter 6 concerns another mechanistic study which describes whether the beneficial effect of calcium on intestinal permeability depends on the functioning of calcium as a carrier of phosphate into the colon. In the colon, phosphate may subsequently increase luminal buffering capacity, which protects against an acidic $\mathrm{pH}$ of the colonic contents due to microbial fermentation, and possibly prevents an increase in intestinal permeability. For this purpose, rats were fed different quantities of calcium and phosphate. CrEDTA was added to the diets to quantify intestinal permeability, and scFOS were supplemented to the different diets to stimulate microbial fermentation and subsequent acidification of the colonic contents. The results of this study show that the beneficial effect of dietary calcium on intestinal permeability was impaired if dietary phosphate levels were low. The protective effect of calcium was associated with a calcium-phosphate-induced increase in luminal buffering capacity. The increase in buffering capacity might therefore be responsible for the effect of calcium on intestinal permeability.

In conclusion, nutritional modulation of intestinal inflammation is possible, at least in rats, as is shown in this thesis for calcium and heme. Most importantly, it is shown for the first time that dietary calcium in rats, besides being protective against intestinal infection, is also able to attenuate chronic intestinal inflammation, as in IBD. It shows the usefulness of an easily applicable nutritional intervention in the modulation of intestinal inflammation. Now the protective effect of supplemental calcium should be validated in the human situation. 
| Summary 
Samenvatting 
Er zijn miljoenen mensen wereldwijd met een chronische ontsteking van het maagdarmkanaal. Zij lijden aan een inflammatoire darmziekte, wat in het Engels wordt aangeduid met inflammatory bowel disease (IBD). De twee hoofdvormen van IBD zijn colitis ulcerosa en de ziekte van Crohn. De oorzaak en het ziekteproces van IBD zijn grotendeels onbekend. Volgens de meest gangbare hypothese resulteert het ontstekingsproces in de darm uit het disfunctioneren van de barrièrefunctie van de darmmucosa, de microbiële factoren in de darm en een verstoorde reactiviteit van het immuunsysteem in een genetisch gevoelige persoon (Figuur 1 en 2). Deze determinanten spelen niet alleen een rol bij het ontstaan van darmontsteking, maar zijn ook van belang voor darmgezondheid in het algemeen. Omgevingsfactoren, zoals voeding, kunnen de bovengenoemde determinanten van de ontwikkeling van IBD beïnvloeden. Echter, onderzoek naar de modulatie van IBD door middel van voeding staat nog in de kinderschoenen. Daarom is de beïnvloeding van darmontsteking door voeding onderzocht in dit proefschrift.

In hoofdstuk 2 van dit proefschrift is het effect van heem in de voeding op darmontsteking onderzocht. Heem is de voedingscomponent die verantwoordelijk is voor de rode kleur van rood vlees. Heem wordt enerzijds beschouwd als een schadelijke factor binnen het onderzoek naar dikke darmkanker. Anderzijds zou de irritatie die door heem wordt veroorzaakt in de holte van de dikke darm (colon) kunnen leiden tot het stimuleren van mogelijk beschermende 'heat shock' eiwitten. Om dit effect van heem te onderzoeken werden ratten gevoed met een gehumaniseerd westers dieet met of zonder heem, waarna colitis (colonontsteking) chemisch werd opgewekt. De resultaten van deze dierstudie tonen aan dat heem in de voeding colitis nadelig beïnvloedt, ondanks het feit dat heem inderdaad de 'heat shock' eiwitten stimuleert in de darmwand. Met name het herstel van het lichaamsgewicht van de dieren, de lengte van het colon en het voerverbruik waren lager in ratten die het heem-voer kregen en dat zijn ongunstige tekenen. Er wordt gespeculeerd dat de celbeschadigende componenten die door heem worden voortgebracht in de holte van de dikke darm het herstel van de darmmucosa remmen. Dit verergert vervolgens de ernst van de colitis. Daarom zou een voeding die veel rood vlees bevat mogelijk een risicofactor zijn voor het krijgen van IBD.

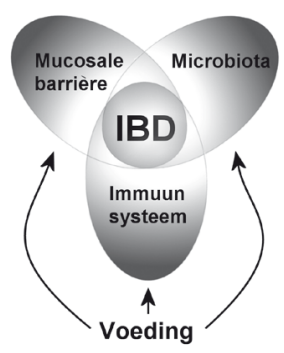

Figuur 1: De drie verschillende determinanten van IBD kunnen beïnloed worden door voeding 
Hoofdstuk 3 beschrijft het effect van calciumsuppletie op darmontsteking. Het is eerder aangetoond dat de toevoeging van calcium aan de voeding de barrièrefunctie van de darmmucosa versterkt, wat ook van belang zou kunnen zijn in IBD. Daarom zijn HLA-B27 transgene ratten, die spontaan colitis krijgen, gevoed met een gehumaniseerd westers voer dat ofwel een laag ofwel een hoog calciumgehalte bevatte. Dit dierexperiment laat zien dat het hoog calcium-voer de ongewenste toename in darmdoorlaatbaarheid en de ernst van de diarree remt tijdens de ontwikkeling van colitis in de HLA-B27 ratten. Bovendien voorkomt calciumsuppletie de toename van de expressie van extracellulaire matrix remodelleringsgenen zoals gewoonlijk tijdens colitis plaatsvindt. Dus calcium in de voeding vermindert enkele belangrijke symptomen en beïnvloedt mechanismes in de ontwikkeling van colitis. Deze resultaten zijn veelbelovend voor calciumsuppletie als effectieve aanvullende therapie voor IBD patiënten, naast de medicatie die nodig zal blijven.

Het doel van hoofdstuk $\mathbf{4}$ was om te onderzoeken of suppletie met een antioxidantenmix het colitisverloop zou verbeteren en of de combinatie van deze antioxidantenmix samen met calcium extra beschermend zou werken. In de literatuur wordt namelijk gesuggereerd dat oxidatieve stress een belangrijke rol speelt in de ontwikkeling van darmontsteking en antioxidanten zouden oxidatieve stress kunnen verminderen. Voor deze proef werden HLA-B27 ratten gevoed met een gehumaniseerd westers controlevoer, of een voer aangevuld met de antioxidanten glutathion, vitamine $\mathrm{C}$ en vitamine $\mathrm{E}$, of een voer aangevuld met zowel deze antioxidantenmix als calcium. De resultaten van hoofdstuk 4 laten zien dat suppletie met de antioxidantenmix geen enkel effect heeft in HLA-B27 ratten ondanks heel lage glutathionniveaus in hun colonmucosa. Echter, de ontwikkeling van colitis ging niet gepaard met een verhoogde mate van oxidatieve stress in dit diermodel. Hieruit kan geconcludeerd worden dat het HLA-B27 transgene ratmodel geen geschikt model is om het effect van antioxidantsuppletie te bestuderen. Omdat de toevoeging van zowel calcium als de antioxidantenmix aan het voer resulteerde in een minder ernstig verloop van de colitis, wordt dit beschermende

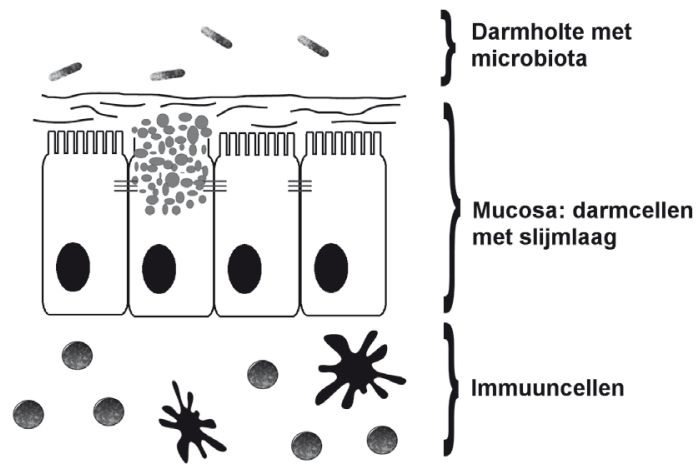

Figuur 2: Schematische weergave van de darm 
effect toegeschreven aan de calciumsuppletie alleen. Het beschermende effect van calciumsuppletie op darmontsteking uit het onderzoek in hoofdstuk $\mathbf{3}$ wordt dus bevestigd door deze studie.

Er wordt gesuggereerd dat een verlaging van de darmdoorlaatbaarheid een belangrijke rol speelt in het beschermende effect van calcium op darmontsteking. Een hoge darmd oorlaatbaarheid wordt namelijk als ongunstig beschouwd. Daarom is in hoofdstuk 5 een meer mechanistische studie gedaan, waarin onderzocht werd hoe en waar calcium in het maag-darmkanaal zijn invloed uitoefent op de darmdoorlaatbaarheid. Om dit te kunnen bestuderen werd een gehumaniseerde westerse controlevoeding gegeven aan ratten, of een voeding waaraan calcium was toegevoegd. Ook de lokalisatie van het effect op darmdoorlaatbaarheid door fructo-oligosacchariden werd bestudeerd in dit experiment. Deze zogenaamde prebiotische vezels worden gefermenteerd door de microbiota in de darm. Lactulose, mannitol en CrEDTA werden toegevoegd aan de voeren om de doorlaatbaarheid van de dikke en dunne darm apart te kunnen kwantificeren. Dit dierexperiment laat zien dat zowel calcium als fructooligosacchariden alleen de doorlaatbaarheid van het colon beïnvloeden en niet de doorlaatbaarheid van de dunne darm: calcium verlaagt, terwijl fructo-oligosacchariden de colondoorlaatbaarheid verhogen. Bovendien beïnvloeden de fecale waters, verkregen uit het voedingsinterventie-experiment, de colondoorlaatbaarheid in (ex-vivo) Ussing kamers niet. Daarom wordt gesuggereerd dat de modulatie van de slijmlaag van de darmwand en/of de microbiota van belang is voor het in vivo effect van calcium en fructo-oligosacchariden in de voeding.

Hoofdstuk 6 betreft een andere mechanistische studie waarin werd onderzocht of het gunstige effect van calcium op darmdoorlaatbaarheid afhankelijk is van het functioneren van calcium als een transporteur (carrier) van fosfaat naar het colon. Vervolgens zou fosfaat in de colonholte de buffercapaciteit kunnen verhogen, wat beschermt tegen een lage $\mathrm{pH}$ van de coloninhoud veroorzaakt door microbiële fermentatie. Dit remmen van de verzuring voorkomt mogelijk dat de darmmucosa schade oploopt waardoor de darmdoorlaatbaarheid toe zou kunnen nemen. Voor dit experiment werden ratten gevoerd met verschillende hoeveelheden calcium en fosfaat in het gehumaniseerde westerse voer. CrEDTA was toegevoegd aan het voer om de darmdoorlaatbaarheid te meten. Daarnaast werden fructo-oligosacchariden gesuppleerd aan de voeren om microbiële fermentatie te stimuleren, waardoor de coloninhoud zou verzuren. De resultaten van deze studie laten zien dat het beschermende effect van calciumsuppletie op darmdoorlaatbaarheid geremd wordt als het fosfaatniveau in het voer laag is. Het gunstige effect van calcium op de darmdoorlaatbaarheid wordt daarom geassocieerd met een toename van de buffercapaciteit in de colonholte veroorzaakt door calciumfosfaat. De verhoging van de buffercapaciteit zou dus mogelijk verantwoordelijk kunnen zijn voor het effect van calcium op darmdoorlaatbaarheid.

Uit de experimenten van dit proefschrift kan worden geconcludeerd dat het mogelijk is om darmontsteking te beïnvloeden door voeding, wat met name is aangetoond voor calcium en heem en in ieder geval geldt voor ratten. Het meest interessant is de bevinding dat voor het eerst is aangetoond dat calciumsuppletie, naast het beschermende effect tegen darminfecties, ook darmontsteking zoals in IBD kan verlichten. De potentie van 
een gemakkelijk toepasbare voedingsinterventie om darmontsteking te beïnvloeden is hiermee aangetoond. Wil dit echt zijn weg naar een toepassing vinden, dan moet het beschermende effect van calciumsuppletie nu vervolgens nog aangetoond worden in de humane situatie. 


\section{Samenvatting}

1 
Dankwoord 
Op deze plek kan ik eindelijk iedereen enorm bedanken!!! Eén ding is wel duidelijk: een promotieonderzoek kun je niet alleen doen. Zonder collega's, vrienden, familie en kennissen had ik deze promotie nooit tot een goed einde kunnen brengen. Jullie steun, hulp, medewerking, vertrouwen en enthousiasme waren onmisbaar!!!

Bedankt!!! 


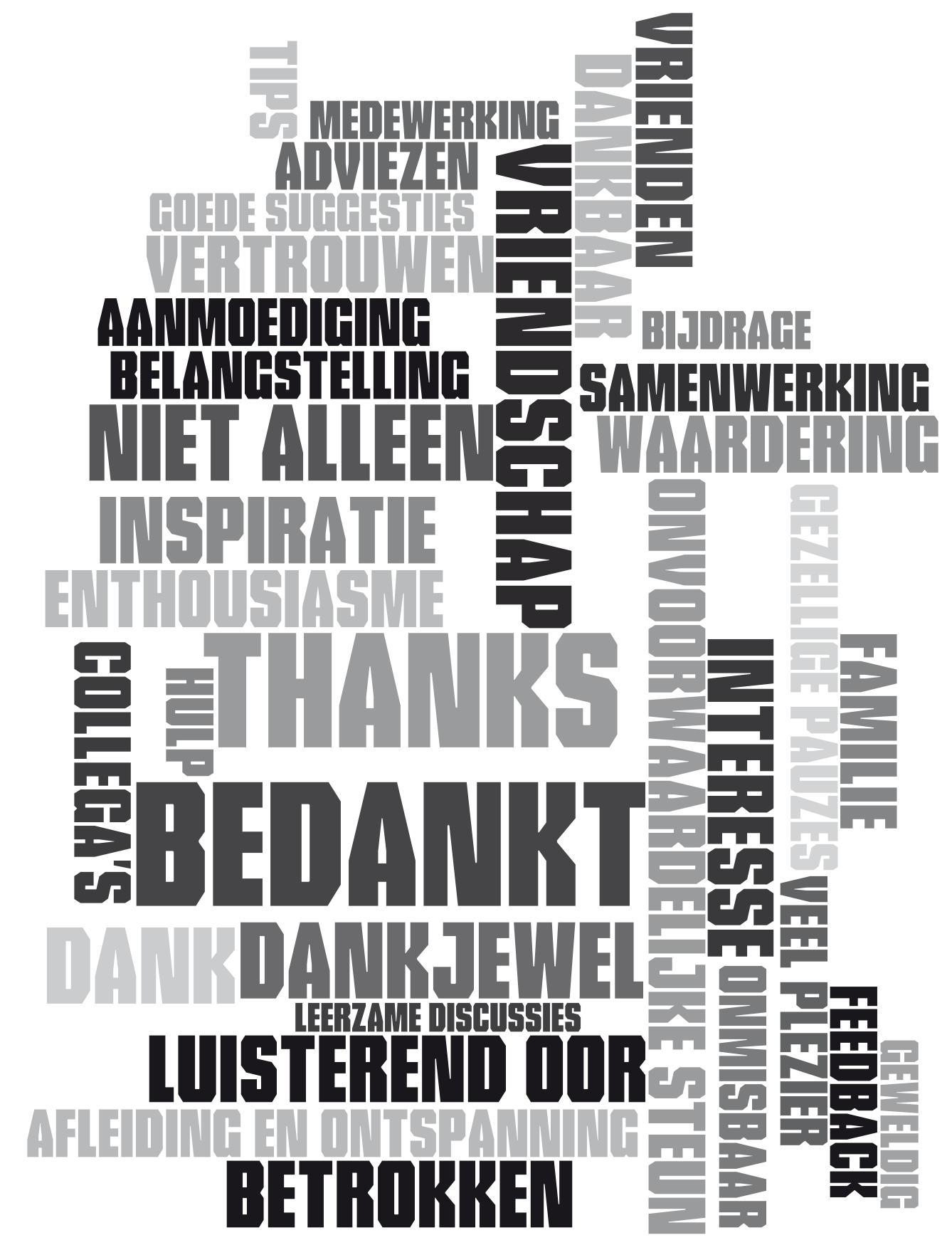


I

| 138 
Publicaties en curriculum vitae 


\section{Publicaties}

Schepens MA, Ten Bruggencate SJ, Schonewille AJ, Brummer R-J, Van der Meer R, and Bovee-Oudenhoven IM. The protective effect of supplemental calcium on colonic permeability depends on a calcium-phosphate-induced increase in luminal buffering capacity. Submitted for publication.

Schepens MA, Vink C, Schonewille AJ , Roelofs HM, Brummer R-J, Van der Meer R, and Bovee-Oudenhoven IM. Supplemental antioxidants do not ameliorate colitis development in HLA-B27 transgenic rats despite extremely low glutathione levels in colonic mucosa. Inflamm Bowel Dis. 2010 Dec 22. In press.

Schepens MA, Rijnierse A, Schonewille AJ, Vink C, Brummer R-J, Willemsen LE, Van der Meer R, and Bovee-Oudenhoven IM. Dietary calcium decreases but short-chain fructooligosaccharides increase colonic permeability in rats. Br J Nutr. 2010 Dec;104(12):17806.

Schepens MA, Vink C, Schonewille AJ, Dijkstra G, Van der Meer R, and Bovee-Oudenhoven IM. Dietary heme adversely affects experimental colitis in rats, despite heat-shock protein induction. Nutrition. 2010 Aug 11. In press.

Schepens MA, Schonewille AJ, Vink C, Van Schothorst EM, Kramer E, Hendriks T, Brummer R-J, Keijer J, Van der Meer R, and Bovee-Oudenhoven IM. Supplemental calcium attenuates the colitis-related increase in diarrhea, intestinal permeability and extracellular matrix breakdown in HLA-B27 transgenic rats. J Nutr. 2009 Aug;139(8):1525-33.

Wigren HK, Schepens M, Matto V, Stenberg D, Porkka-Heiskanen T. Glutamatergic stimulation of the basal forebrain elevates extracellular adenosine and increases the subsequent sleep. Neuroscience. 2007 Jul 13;147(3):811-23.

Schepens MA, Roelofs HM, Peters WH, Wanten GJ. No evidence for oxidative stress in patients on home parenteral nutrition. Clin Nutr. 2006 Dec;25(6):939-48. 


\section{Curriculum vitae}

Marloes Anna Adriana Schepens werd 17 november 1981 geboren te Veghel. In 2000 behaalde zij het gymnasium diploma aan het Zwijsen College te Veghel. Vervolgens studeerde zij Biomedische Wetenschappen aan de Radboud Universiteit Nijmegen. Tijdens de masterfase van deze studie werd een bijvakstage uitgevoerd aan de University of Helsinki in Finland. Hier werd het effect van glutamaat op slaap en lactaat-, pyruvaat- en adenosineniveaus in de hersenen van jonge en oude ratten onderzocht. Haar hoofdvakstage vond plaats bij de afdeling Maag-, Darm- en Leverziekten van het Universitair Medisch Centrum St Radboud te Nijmegen. Tijdens deze stage onderzocht ze de oxidatieve stress status in het bloed van patiënten met totaal parenterale voeding. In 2005 studeerde ze af, waarna ze in hetzelfde jaar haar promotieonderzoek startte dat beschreven staat in dit proefschrift. Dit promotieonderzoek werd uitgevoerd onder begeleiding van Dr. ir. I.M.J. Bovee-Oudenhoven en Prof. dr. R.-J. Brummer. Het promotieproject was onderdeel van het Top Institute Food and Nutrition en vond plaats bij NIZO food research te Ede. 
I

| 142 
I

| 144 